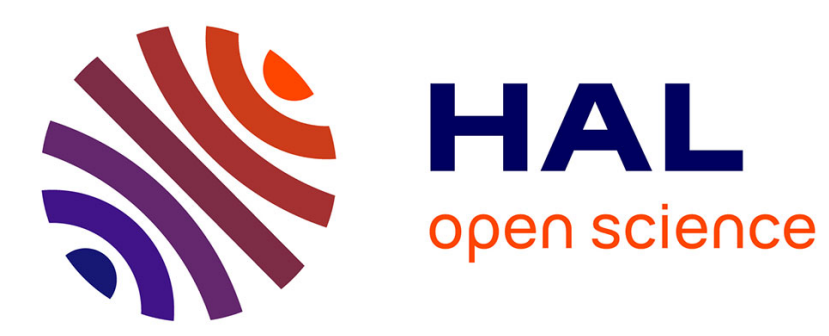

\title{
RFC7939: Definition of Managed Objects for the Neighborhood Discovery Protocol
}

Robert G. Cole, I Chakeres, Delvin T Clausen, Thomas Heide Clausen

\section{To cite this version:}

Robert G. Cole, I Chakeres, Delvin T Clausen, Thomas Heide Clausen. RFC7939: Definition of Managed Objects for the Neighborhood Discovery Protocol. [Technical Report] RFC7939, The Internet Engineering Task Force (IETF). 2016. hal-03172494

HAL Id: hal-03172494

https://hal-polytechnique.archives-ouvertes.fr/hal-03172494

Submitted on 17 Mar 2021

HAL is a multi-disciplinary open access archive for the deposit and dissemination of scientific research documents, whether they are published or not. The documents may come from teaching and research institutions in France or abroad, or from public or private research centers.
L'archive ouverte pluridisciplinaire HAL, est destinée au dépôt et à la diffusion de documents scientifiques de niveau recherche, publiés ou non, émanant des établissements d'enseignement et de recherche français ou étrangers, des laboratoires publics ou privés. 
Internet Engineering Task Force (IETF)

Request for Comments: 7939

Obsoletes: 6779

Category: Standards Track

ISSN : 2070-1721
U. Herberg

R. Cole

US Army CERDEC

I. Chakeres

Delvin

T. Clausen

Ecole Polytechnique

August 2016

Definition of Managed Objects for the Neighborhood Discovery Protocol

Abstract

This document replaces RFC 6779; it contains revisions and extensions to the original document. It defines a portion of the Management Information Base (MIB) for use with network management protocols in the Internet community. In particular, it describes objects for configuring parameters of the Neighborhood Discovery Protocol (NHDP) process on a router. The extensions described in this document add objects and values to support the NHDP optimization specified in RFC 7466. The MIB module defined in this document, denoted NHDP-MIB, also reports state, performance information, and notifications about NHDP. This additional state and performance information is useful to troubleshoot problems and performance issues during neighbor discovery.

Status of This Memo

This is an Internet Standards Track document.

This document is a product of the Internet Engineering Task Force (IETF). It represents the consensus of the IETF community. It has received public review and has been approved for publication by the Internet Engineering Steering Group (IESG). Further information on Internet Standards is available in Section 2 of RFC 7841.

Information about the current status of this document, any errata, and how to provide feedback on it may be obtained at

http://www.rfc-editor.org/info/rfc7939.

Herberg, et al.

RFC 7939
Standards Track

The NHDP-MIB
[Page 1]

August 2016 
Copyright Notice

Copyright (c) 2016 IETF Trust and the persons identified as the document authors. All rights reserved. This document is subject to BCP 78 and the IETF Trust's Legal Provisions Relating to IETF Documents (http://trustee.ietf.org/license-info) in effect on the date of publication of this document. Please review these documents carefully, as they describe your rights and restrictions with respect to this document. Code Components extracted from this document must include Simplified BSD License text as described in section 4.e of the Trust Legal Provisions and are provided without warranty as described in the Simplified BSD License.

\section{Table of Contents}

1. Introduction . . . . . . . . . . . . . . . . . . . 3

1.1. Differences from RFC 6779 ............... 3

2. The Internet-Standard Management Framework . . . . . . . 3

3. Conventions . . . . . . . . . . . . . . . . . . . 4

4. Overview . . . . . . . . . . . . . . . . . . . . 4

4.1. Terms ...................... 4

4.2. Notation ...................... 4

5. Structure of the MIB Module ................ 4

5.1. Notifications .. . . . . . . . . . . . . 5

5.1.1. Introduction . . . . . . . . . . . . . . . . 5

5.1.2. Notification Generation . . . . . . . . . . 5

5.1.3. Limiting Frequency of Notifications . . . . . . . 5

5.2. The Configuration Group . . . . . . . . . . . . . . 7

5.3. The State Group .. . . . . . . . . . . . . . . 7

5.4. The Performance Group . . . . . . . . . . . . . . 8

5.5. Tables and Indexing . . . . . . . . . . . . . . 8

6. Relationship to Other MIB Modules . . . . . . . . . . 10

6.1. Relationship to the SNMPv2-MIB . . . . . . . . . . . . . 10

6.2. Relationship to Routing Protocol MIB Modules Relying on

the NHDP-MIB Module . . . . . . . . . . . . 10

6.3. Relationship to the If-MIB .............. 10

6.4. MIB Modules Required for IMPORTS . . . . . . . . . . . 11

7. Definitions . . . . . . . . . . . . . . . . . . . 11

8. Security Considerations . . . . . . . . . . . . 66

9. Applicability Statement . . . . . . . . . . . . . 68

10. IANA Considerations . . . . . . . . . . . . . . 69

11. References . . . . . . . . . . . . . . . . . 69

11.1. Normative References . . . . . . . . . . . . 69

11.2. Informative References ................. 71

Acknowledgements . . . . . . . . . . . . . . . . 72

Authors' Addresses . . . . . . . . . . . . . 72

Herberg, et al.

RFC 7939

1. Introduction

This document defines a portion of the Management Information Base
[Page 2]

August 2016 
(MIB) for use with network management protocols in the Internet community. In particular, it describes objects for configuring parameters of the Mobile Ad Hoc Network (MANET) Neighborhood Discovery Protocol (NHDP) [RFC6130] process on a router. The MIB module defined in this document, denoted NHDP-MIB, also reports state, performance information, and notifications about NHDP. This additional state and performance information is useful to troubleshoot problems and performance issues during neighbor discovery.

\subsection{Differences from RFC 6779}

This document obsoletes [RFC6779], replacing that document as the specification of the MIB module for [RFC6130]. This revision to [RFC6779] is necessitated by the update to [RFC6130] specified in [RFC7466].

The MIB module for [RFC6130], specified in this document, captures the new information and states for each symmetric 2-hop neighbor, recorded in the Neighbor Information Base of a router and to be reflected in the appropriate tables, introduced by [RFC7466], specifically:

- Addition of objects nhdpIib2HopsetN2Lost and nhdpIfPerfCounterDiscontinuityTime.

o Addition of extra value (notconsidered) to nhdp2HopNbrState.

- Revised full compliance state.

2. The Internet-Standard Management Framework

For a detailed overview of the documents that describe the current Internet-Standard Management Framework, please refer to section 7 of RFC 3410 [RFC3410].

Managed objects are accessed via a virtual information store, termed the Management Information Base or MIB. MIB objects are generally accessed through the Simple Network Management Protocol (SNMP). Objects in the MIB are defined using the mechanisms defined in the Structure of Management Information (SMI). This memo specifies a MIB module that is compliant to the SMIV2, which is described in STD 58, RFC 2578 [RFC2578], STD 58, RFC 2579 [RFC2579] and STD 58, RFC 2580 [RFC2580].

Herberg, et al.

RFC 7939
Standards Track

The NHDP-MIB
[Page 3]

August 2016

3. Conventions

The key words "MUST", "MUST NOT", "REQUIRED", "SHALL", "ShALL NOT", "SHOULD", "SHOULD NOT", "RECOMMENDED", "NOT RECOMMENDED", "MAY", and OPTIONAL" in this document are to be interpreted as described in [RFC2119].

4. Overview 
[RFC6130] allows a router to discover and track topological information of routers up to two hops away by virtue of exchanging HELLO messages. This information is useful for routers running various routing and multicast flooding protocols developed within the IETF MANET Working Group.

4.1. Terms

The following definitions apply throughout this document:

o Notification Objects - triggers and associated notification messages allowing for asynchronous tracking of predefined events on the managed router.

o Configuration objects - switches, tables, and objects that are initialized to default settings or set through the management interface defined by this MIB module.

- State Objects - automatically generated values that define the current operating state of the NHDP instance in the router.

- Performance objects - automatically generated values that help to assess the performance of the NHDP instance on the router and the overall discovery performance within the MANET.

\subsection{Notation}

The same notations as defined in [RFC6130] are used throughout this document.

5. Structure of the MIB Module

This section presents the structure of the NHDP-MIB module. The MIB module is arranged into the following structure:

o nhdpNotifications - objects defining NHDP-MIB notifications.

Herberg, et al.

RFC 7939
Standards Track

The NHDP-MIB
[Page 4]

August 2016

o nhdpobjects - defining objects within this MIB module. The objects are arranged into the following groups:

* Configuration Group - defining objects related to the configuration of the NHDP instance on the router.

* State Group - defining objects that reflect the current state of the NHDP instance running on the router.

* Performance Group - defining objects that are useful to a management station when characterizing the performance of NHDP on the router and in the MANET. 
- nhdpconformance - defining the minimal and maximal conformance requirements for implementations of this MIB module.

\subsection{Notifications}

This section describes the use of notifications and mechanisms to enhance the ability to manage NHDP routing domains.

5.1.1. Introduction

Notifications can be emitted by a router running an instance of this specification as a reaction to a specific event. This allows an observer of these events to efficiently determine the source of problems or significant changes of configuration or topology, instead of polling a possibly large number of routers.

\subsubsection{Notification Generation}

When an exception event occurs, the application notifies the local agent, which sends a notification to the appropriate SNMP management stations. The message includes the notification type and may include a list of notification-specific variables. Section 7 contains the notification definitions, which includes the variable lists. At least one IP address of the router that originates the notification is included in the variable list so that the source of the notification may be determined.

\subsubsection{Limiting Frequency of Notifications}

To limit the frequency of notifications, the following additional mechanisms are suggested, similar to those in [RFC4750].

Herberg, et al.

RFC 7939
Standards Track

The NHDP-MIB
[Page 5]

August 2016

\subsubsection{Ignoring Initial Activity}

The majority of critical events occur when NHDP is first enabled on a router, at which time, the symmetric neighbors and 2-hop neighbors of the router are discovered. During this initial period, a potential flood of notifications is unnecessary since the events are expected. To avoid unnecessary notifications, a router SHOULD NOT originate expected notifications until a predefined and administratively configured time interval has elapsed. It is RECOMMENDED that this time interval be at least 3 times nhdpHelloInterval so that symmetric neighbors are discovered. The suppression window for notifications is started when the nhdpIfstatus transitions from its default value of 'false(2)' to 'true(1)'.

\subsubsection{Throttling Notifications}

The mechanism for throttling the notifications is the same as in [RFC4750] (i.e., the number of transmitted notifications per time is 
bounded )

Appropriate values for the window time and upper bound are to be administratively configured and depend on the deployment of the MANET. If NHDP is deployed on a lossy, wireless medium, sending too many notifications in a short time interval may lead to collisions and dropped packets. In particular, in dense deployments of routers running NHDP (i.e., where each router has many neighbors), a change of the local topology may trigger many notifications at the same time. [RFC4750] recommends "7 traps with a window time of 10 seconds" as the upper bound. As NHDP is expected to be deployed in more lossy channels than OSPF, it is RECOMMENDED to choose a lower threshold for the number of notifications per time than that. Specifically, it is RECOMMENDED that the threshold value for the objects reflecting the change be set to a value of '10' and the DEFAULT values for these objects within the Notifications Group be set to this value. Further, a time window for the change objects is defined within this MIB module. If the number of occurrences exceeds the change threshold within the previous change window, then it is RECOMMENDED that the notification be sent. Furthermore, it is RECOMMENDED that the value for this window be set to at least 5 times the nhdpHelloInterval.

The following objects are used to define the thresholds and time windows for specific notifications defined in the NHDP-MIB module: nhdpNbrStatechangeThreshold, nhdpNbrStatechangewindow, nhdp2HopNbrStateChangeThreshold, and nhdp2HopNbrStateChangeWindow.

Herberg, et al.

RFC 7939
Standards Track

[Page 6]

The NHDP-MIB

August 2016

\subsubsection{One Notification per Event}

Similar to the mechanism in [RFC4750], only one notification is sent per event.

\subsection{The Configuration Group}

The router running NHDP is configured with a set of controls. The authoritative list of configuration controls within the NHDP-MIB module are found within the MIB module itself. Generally, an attempt was made in developing the NHDP-MIB module to support all configuration objects defined in [RFC6130]. For all of the configuration parameters, the same constraints and default values of these parameters as defined in [RFC6130] are followed. Refer to [RFC5148] for guidance on setting jitter-related parameters, e.g., nhdpMaxJitter.

\subsection{The State Group}

The State Group reports current state information of a router running NHDP. The NHDP-MIB State Group tables were designed to contain the complete set of state information defined within the information bases specified in sections 6, 7, and 8 of [RFC6130]. 
Two constructs, i.e., TEXTUAL-CONVENTIONs, are defined to support the tables in the State Group. NHDP stores and indexes information through sets of (dynamically defined) addresses, i.e., address sets. Within SMIv2, it is not possible to index tables with variably defined address sets. Hence, these TEXTUAL-CONVENTIONs are defined to provide a local mapping between NHDP-managed address sets and SMIv2 table indexing. These constructs are the NeighborIfIndex and NeighborRouterIndex. These are locally (to the router) defined, unique identifiers of virtual neighbors and neighbor interfaces. Due to the nature of NHDP, the local router may have identified distinct address sets but is not able to associate these as a single interface. Hence, two or more NeighborIfIndexes pointing to multiple distinct address sets may, in fact, be related to a common neighbor interface. This ambiguity may also hold with respect to the assignment of the NeighborRouterIndex. The local MIB agent is responsible for managing, aggregating, and retiring the defined indexes and for updating MIB tables using these indexes as the local router learns more about its neighbors' topologies. These constructs are used to define indexes to the appropriate State Group tables and to correlate table entries to address sets, virtual neighbor interfaces, and virtual neighbors within the MANET.

Herberg, et al.

RFC 7939
Standards Track

The NHDP-MIB
[Page 7]

August 2016

\subsection{The Performance Group}

The Performance Group reports values relevant to system performance. Unstable neighbors or 2-hop neighbors and frequent changes of sets can have a negative influence on the performance of NHDP. This MIB module defines several objects that can be polled in order to, e.g., calculate histories or monitor frequencies of changes. This may help an observer determining unusual topology changes or other changes that affect stability and reliability of the MANET.

\subsection{Tables and Indexing}

The NHDP-MIB module contains a number of tables that record data related to:

o the local router,

- a local MANET interface on the router,

o other routers that are one hop removed from the local router,

o interfaces on other routers that are one hop removed from the local router, and

o other routers that are two hops removed from the local router.

The NHDP-MIB module's tables are indexed via the following constructs : 
o nhdpIfIndex - the IfIndex of the local router on which NHDP is configured.

- nhdpDiscIfIndex - a locally managed index representing a known interface on a neighboring router.

o nhdpDiscRouterIndex - a locally managed index representing an ID of a known neighboring router.

These tables and their indexing are:

- nhdpInterfaceTable - describes the configuration of the interfaces of this router. This table has INDEX $\{$ nhdpIfIndex $\}$.

o nhdpLibLocalifSetTable - records all network addresses that are defined as local interface network addresses on this router. This table has INDEX \{ nhdpLibLocalIfSetIndex $\}$.

Herberg, et al.

RFC 7939
Standards Track

The NHDP-MIB
[Page 8]

August 2016

o nhdpLibRemovedIfAddrSetTable - records network addresses that were recently used as local interface network addresses on this router but have been removed. This table has INDEX \{ nhdpLibRemovedIfAddrSetIndex $\}$.

- nhdpInterfacestateTable - records state information related to specific interfaces of this router. This table has INDEX $\{$ nhdpIfIndex $\}$.

o nhdpDiscIfSetTable - includes the nhdpDiscRouterIndex of the discovered router, the nhdpDiscIfIndex of the discovered interface, and the current set of addresses associated with this neighbor interface. This table has INDEX \{ nhdpDiscIfSetIndex .

o nhdpIibLinkSetTable - for each local interface, records all links belonging to other routers that are, or recently were, 1-hop neighbors to this router. This table has INDEX \{ nhdpIfIndex, nhdpDiscIfIndex $\}$.

o nhdpIib2HopSetTable - for each local interface, records network addresses (one at a time) of symmetric 2-hop neighbors and the symmetric links to symmetric 1-hop neighbors of this router through which these symmetric 2-hop neighbors can be reached. This table has INDEX \{ nhdpIfIndex, nhdpDiscIfIndex, nhdpIib2HopSetIpAddressType, nhdpIib2HopSetIpAddress \} .

- nhdpNibNeighborSetTable - records all network addresses of each 1-hop neighbor to this router. This table has INDEX

\{ nhdpDiscRouterIndex $\}$.

o nhdpNibLostNeighborSetTable - records network addresses of other routers that were recently symmetric 1-hop neighbors to this router but are now advertised as lost. This table has INDEX 
$\{$ nhdpDiscRouterIndex $\}$.

- nhdpInterfacePerfTable - records performance objects that are measured for each local NHDP interface on this router. This table has INDEX \{ nhdpIfIndex $\}$.

- nhdpDiscIfSetPerfTable - records performance objects that are measured for each discovered interface of a neighbor of this router. This table has INDEX $\{$ nhdpDiscIfIndex $\}$.

o nhdpDiscNeighborSetPerfTable - records performance objects that are measured for discovered neighbors of this router. This table has INDEX \{ nhdpDiscRouterIndex $\}$.

Herberg, et al.

RFC 7939
Standards Track

The NHDP-MIB
[ Page 9]

August 2016

- nhdpIib2HopsetPerfTable - records performance objects that are measured for discovered 2-hop neighbors of this router. This table has INDEX \{ nhdpDiscRouterIndex $\}$.

6. Relationship to Other MIB Modules

This section specifies the relationship of the MIB module contained in this document to other standards, particularly to standards containing other MIB modules. MIB modules and specific definitions imported from MIB modules that SHOULD be implemented in conjunction with the MIB module contained within this document are identified in this section.

6.1. Relationship to the SNMPv2-MIB

The System Group in the SNMPV2-MIB module [RFC3418] is defined as being mandatory for all systems, and the objects apply to the entity as a whole. The System Group provides identification of the management entity and certain other system-wide data. The NHDP-MIB module does not duplicate those objects.

6.2. Relationship to Routing Protocol MIB Modules Relying on the NHDPMIB Module

[RFC6130] allows routing protocols to rely on the neighborhood information that is discovered by means of HELLO message exchange. In order to allow for troubleshooting, fault isolation, and management of such routing protocols through a routing protocol MIB module, it may be desired to align the state Group tables of the NHDP-MIB module and the routing protocol MIB module. This is accomplished through the definition of two TEXTUAL-CONVENTIONs in the NHDP-MIB module: the NeighborIfIndex and the NeighborRouterIndex. These object types are used to develop indexes into common NHDP-MIB module and routing protocol state Group tables. These objects are locally significant but should be locally common to the NHDP-MIB module and the routing protocol MIB module implemented on a common networked router. This will allow for improved cross-referencing of information across the two MIB modules. 


\subsection{Relationship to the If-MIB}

The nhdpInterfaceTable in this MIB module describes the configuration of the interfaces of this router that are intended to use MANET control protocols. As such, this table 'sparse augments' the ifTable [RFC2863] specifically when NHDP is to be configured to operate over this interface. The interface is identified by the ifIndex from the Interfaces Group defined in the Interfaces Group MIB module [RFC2863].

Herberg, et al.

RFC 7939
Standards Track

The NHDP-MIB
[Page 10]

August 2016

A conceptual row in the nhdpInterfaceTable exists if and only if either the row has been administratively created or there is an interface on the managed device that supports and runs NHDP. This implies that for each entry in the nhdpInterfaceTable, there is a corresponding entry in the Interface Table where nhdpIfIndex and ifIndex are equal. If that corresponding entry in the Interface Table is deleted, then the entry in nhdpInterfaceTable is automatically deleted, NHDP is disabled on this interface, and all configuration and state information related to this interface is to be removed from memory.

\subsection{MIB Modules Required for IMPORTS}

The following NHDP-MIB module IMPORTS objects from SNMPv2-SMI [RFC2578], SNMPv2-TC [RFC2579], SNMPv2-CONF [RFC2580], IF-MIB [RFC2863], SNMP-FRAMEWORK-MIB [RFC3411], INET-ADDRESS-MIB [RFC4001], and FLOAT-TC-MIB [RFC6340].

7. Definitions

This section contains the MIB module defined by the specification.

NHDP-MIB DEFINITIONS : := BEGIN

-- This MIB module defines objects for the management of

-- NHDP (RFC 6130) - Mobile Ad Hoc Network (MANET)

-- Neighborhood Discovery Protocol (NHDP),

-- Clausen, T., Dearlove, C., and J. Dean, January 2011.

IMPORTS

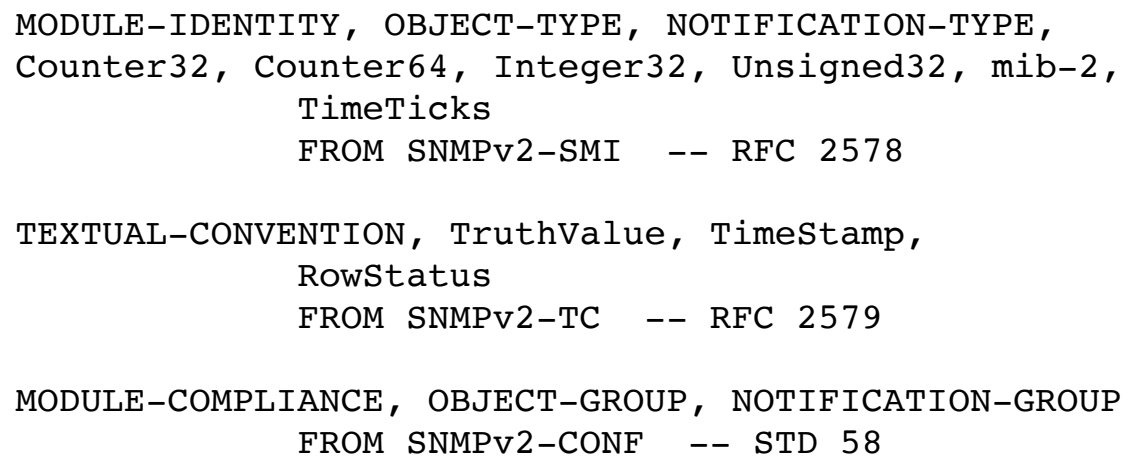


SnmpAdminstring

FROM SNMP-FRAMEWORK-MIB -- RFC 3411

Herberg, et al.

RFC 7939
Standards Track

The NHDP-MIB
[Page 11]

August 2016

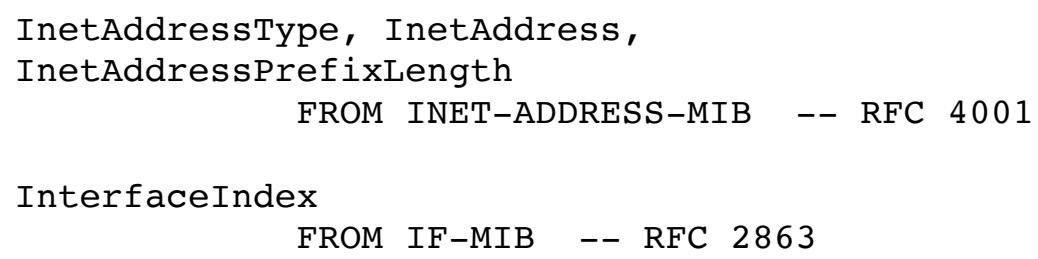


Herberg, et al.

RFC 7939
Standards Track

The NHDP-MIB
[Page 12]

August 2016

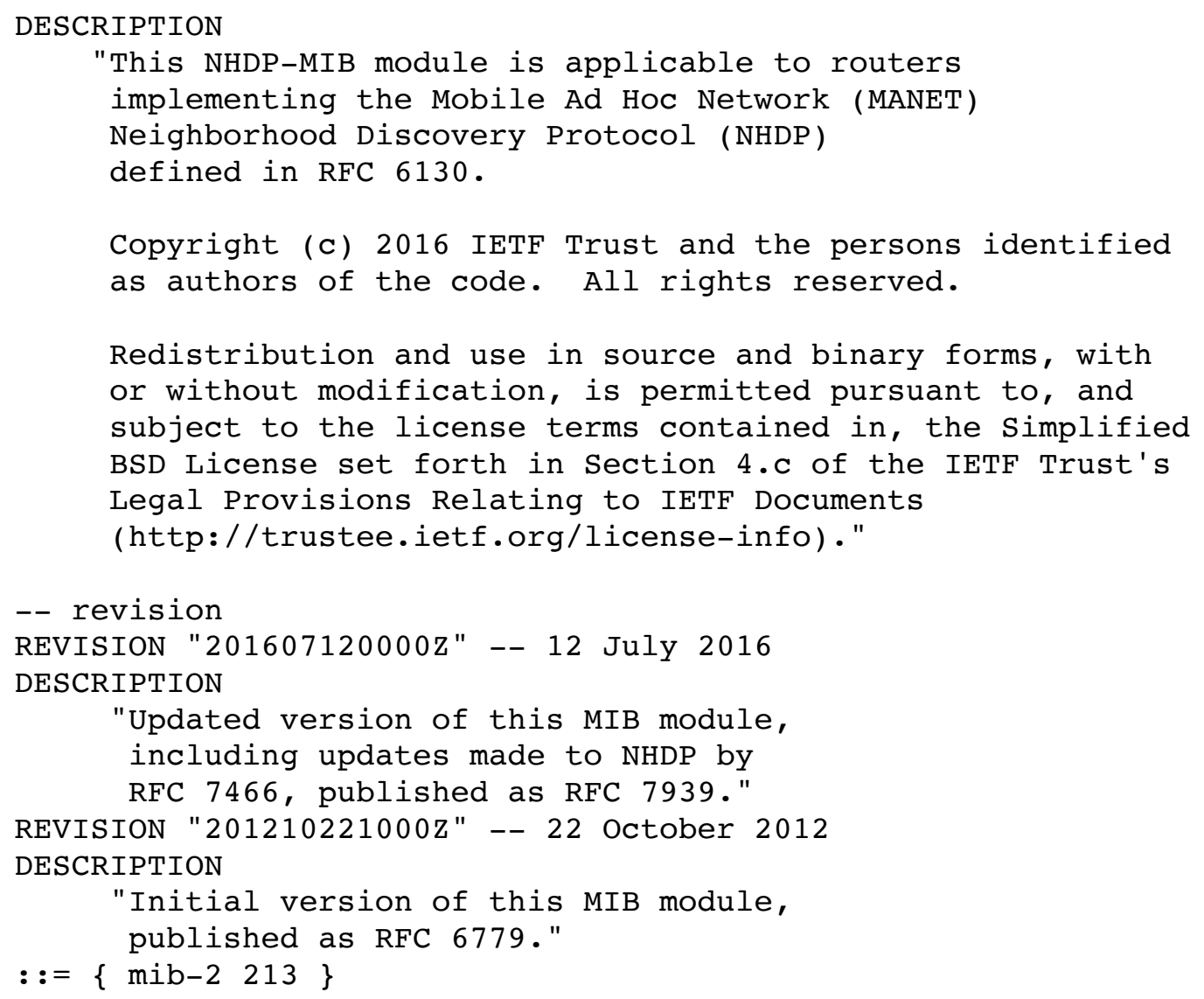

-- Top-Level Components of this MIB Module

$--$

nhdpNotifications OBJECT IDENTIFIER ::= $\left.\begin{array}{lll}\text { nhdpMIB } & 0\end{array}\right\}$

nhdpobjects OBJECT IDENTIFIER ::= $\operatorname{nhdpMIB} 1\}$

nhdpConformance OBJECT IDENTIFIER ::= $\left\{\begin{array}{ll}\text { nhdpMIB } & 2\end{array}\right\}$

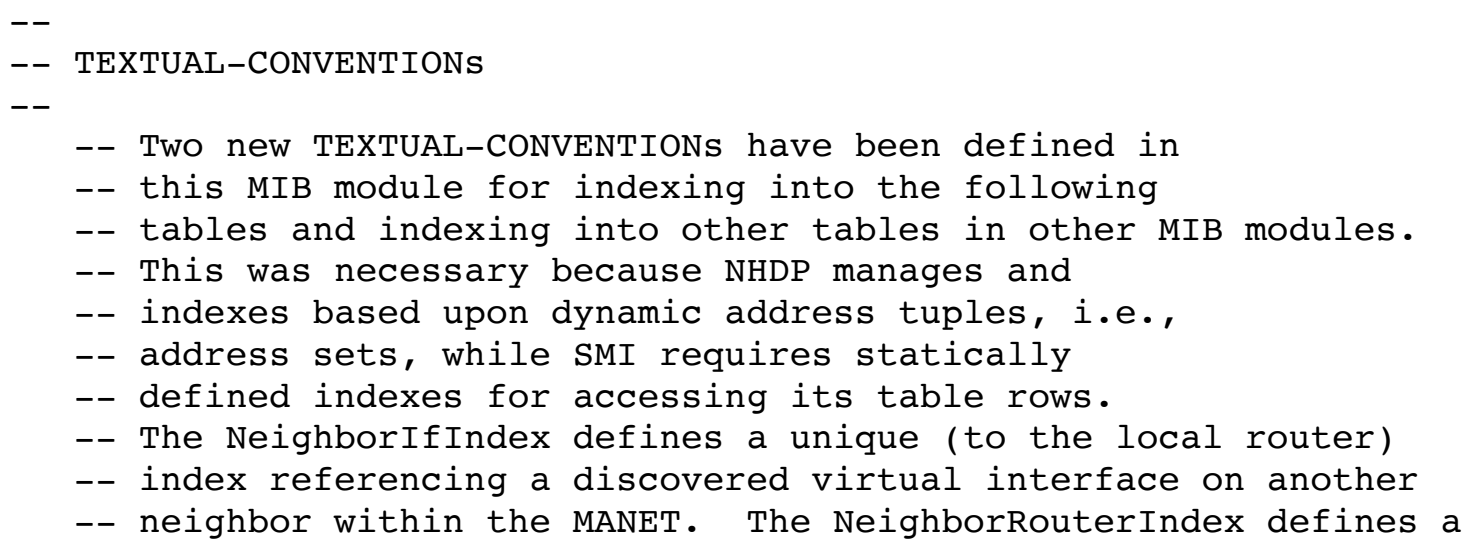


-- unique (to the local router) index referencing a discovered

-- virtual neighbor within the MANET.

$--$

-- Due to the nature of NHDP,

-- different indexes may be related to common neighbor

-- interfaces or common neighbor routers, but the information

-- obtained through NHDP has not allowed the local router

-- to relate these virtual objects (i.e., interfaces or routers)

-- at this point in time. As more topology information

-- is gathered by the local router, it may associate

-- virtual interfaces or routers and collapse these

-- indexes appropriately.

-- Multiple addresses can be associated with a

-- given NeighborIfIndex. Each NeighborIfIndex is

-- associated with a NeighborRouterIndex. Throughout

-- the nhdpstateObjGroup, the

-- NeighborIfIndex and the NeighborRouterIndex are used

-- to define the set of IP Addresses related to a virtual

-- neighbor interface or virtual neighbor under discussion.

\section{NeighborIfIndex : := TEXTUAL-CONVENTION DISPLAY-HINT "d" \\ STATUS DESCRIPTION}

"An arbitrary, locally unique identifier associated with a virtual interface of a discovered NHDP neighbor. Due to the nature of NHDP, the local router may not know if two distinct addresses belong to the same interface of a neighbor or to two different interfaces. As the local router gains more knowledge of its neighbors, its local view may change, and this table will be updated to reflect the local router's current understanding, associating address sets to neighbor interfaces. The local router identifies a virtual neighbor interface through the receipt of address lists advertised through an NHDP HELLO message.

All objects of type NeighborIfIndex are assigned by the agent out of a common number space.

The value for each discovered virtual neighbor interface may not remain constant from one re-initialization of the entity's network management agent to the next re-initialization. If the local router gains information associating two virtual interfaces on a neighbor as a common interface,

Herberg, et al.

RFC 7939
Standards Track

The NHDP-MIB
[Page 14]

August 2016

then the agent MUST aggregate the two address sets to a single index chosen from the set of aggregated indexes, 
and it MUST update all tables in this

MIB module that are indexed by indexes

of type NeighborIfIndex. It MAY then reuse freed

index values following the next agent restart.

The specific value is meaningful only within a given SNMP entity."

SYNTAX Unsigned32 (1..2147483647)

NeighborRouterIndex : := TEXTUAL-CONVENTION

DISPLAY-HINT "d"

STATUS current

DESCRIPTION

"An arbitrary, locally unique identifier associated with a virtual discovered neighbor (one or two hop). Due to the nature of NHDP, the local router may identify multiple virtual neighbors that, in fact, are one and the same. Neighbors that are two hops away with more than one advertised address will exhibit this behavior. As the local router's knowledge of its neighbors' topology increases, the local router will be able to associate multiple virtual neighbor indexes into a single virtual neighbor index chosen from the set of aggregated indexes; it MUST update all tables in this MIB module indexed by these indexes, and it MAY reuse the freed indexes following the next agent re-initialization.

All objects of type NeighborRouterIndex are assigned by the agent out of a common number space.

The NeighborRouterIndex defines a discovered NHDP peer virtual neighbor of the local router. The value for each discovered virtual neighbor index MUST remain constant at least from one re-initialization of the entity's network management agent to the next re-initialization, except if an application is deleted and re-created.

The specific value is meaningful only within a given SNMP entity. A NeighborRouterIndex value MUST NOT be reused until the next agent restart."

SYNTAX Unsigned32 (1..2147483647)

Herberg, et al.

RFC 7939

- nhdpobjects
Standards Track

The NHDP-MIB
[Page 15]

August 2016

1) Configuration Objects Group

2) State Objects Group

3) Performance Objects Group 
-- nhdpConfigurationObjGrp

$--$

-- Contains the NHDP objects that configure specific options

-- that determine the overall performance and operation of

-- NHDP.

nhdpConfigurationobjGrp OBJECT IDENTIFIER ::=\{ nhdpobjects 1$\}$

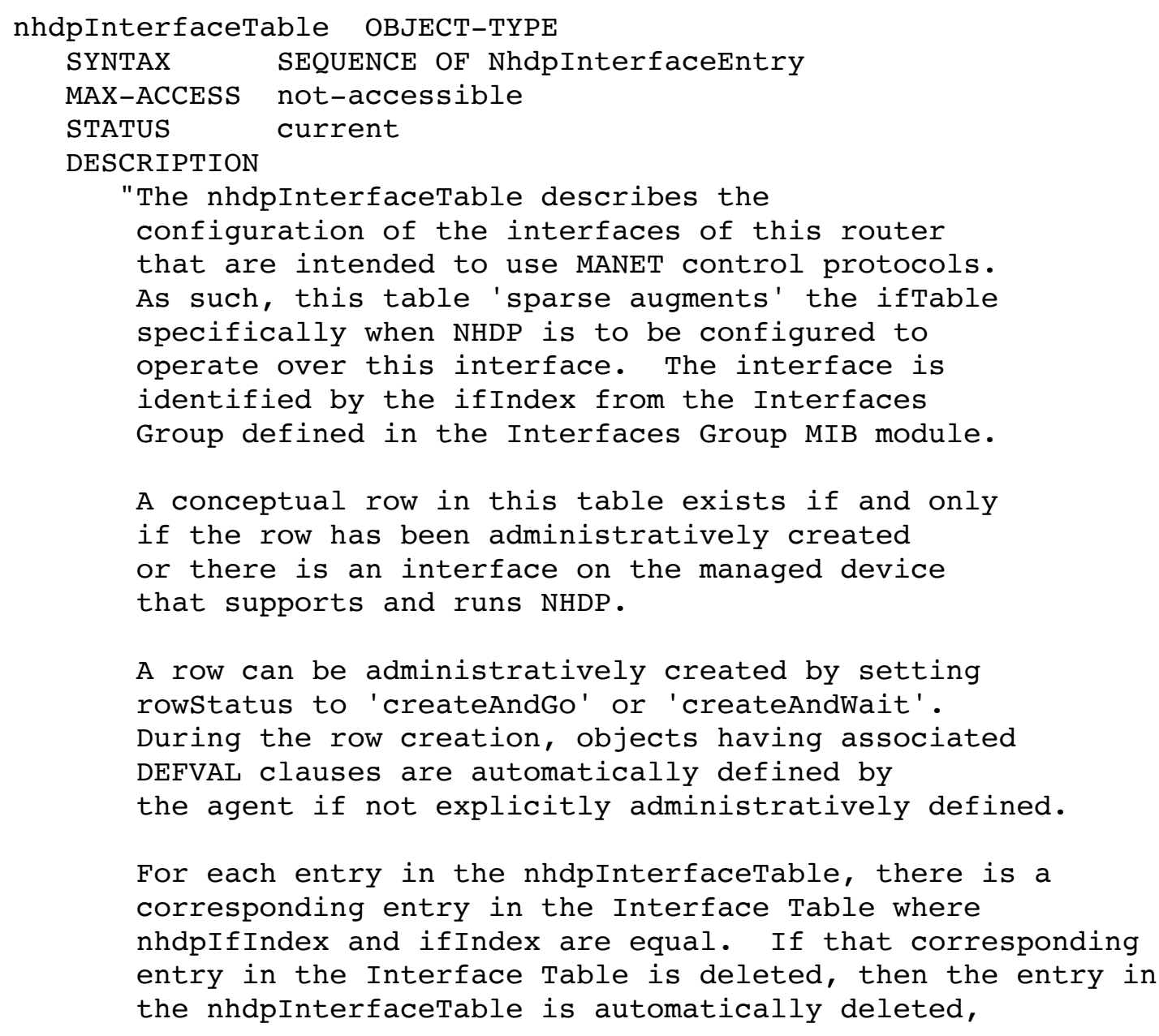

Herberg, et al.

RFC 7939
Standards Track

The NHDP-MIB
[Page 16]

August 2016

\footnotetext{
NHDP is disabled on this interface, and all configuration and state information related to this interface is to be removed from memory." REFERENCE

"RFC 2863 - The Interfaces Group MIB, MCCloghrie, K., and F. Kastenholtz, June 2000"

$::=\{$ nhdpConfigurationobjGrp 1$\}$

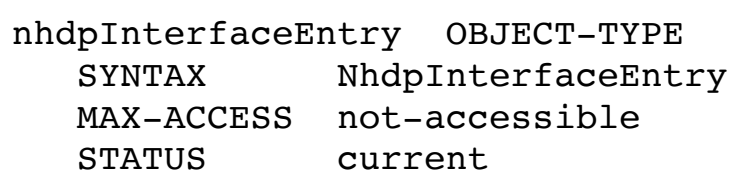




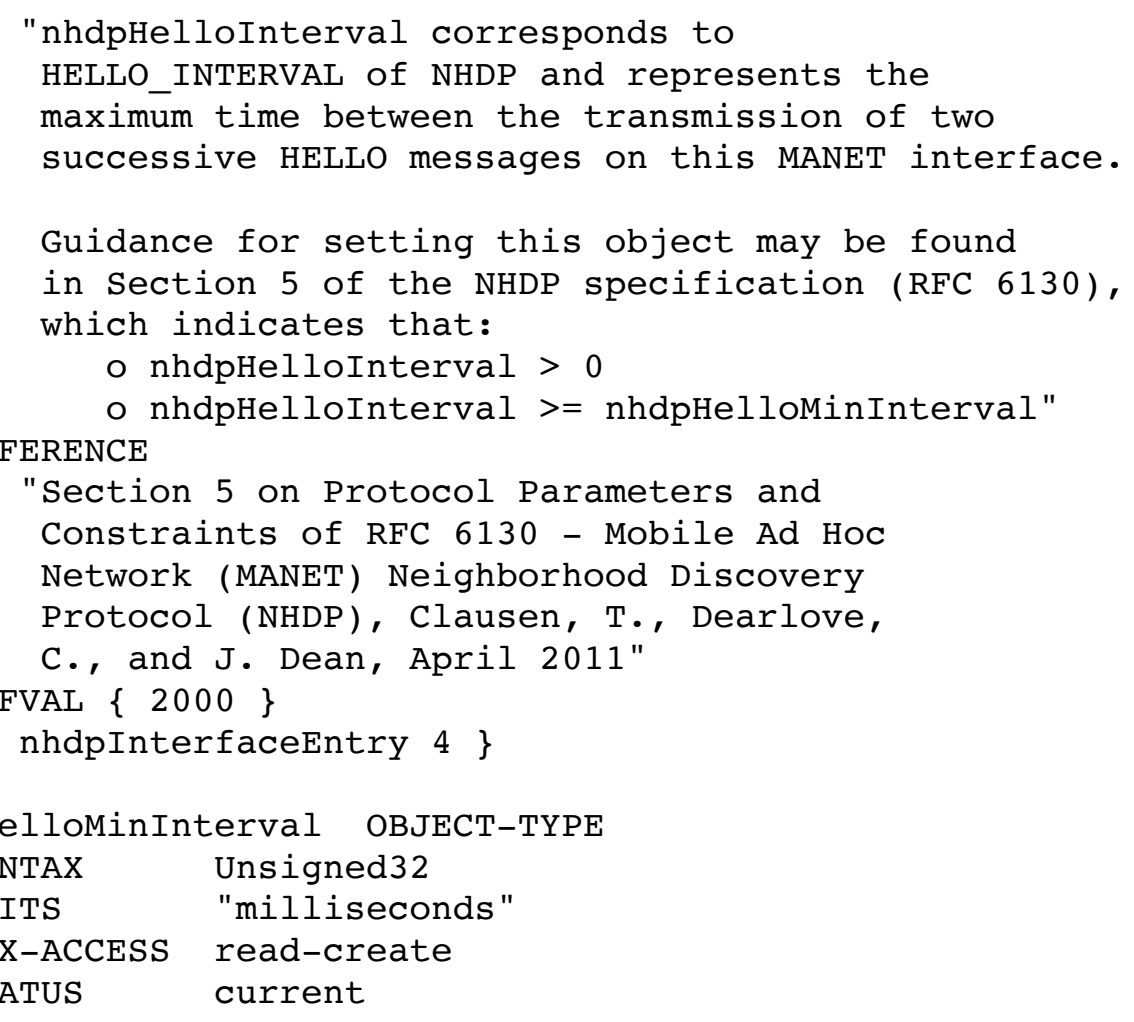

Herberg, et al.

RFC 7939
Standards Track

The NHDP-MIB
[Page 19]

August 2016

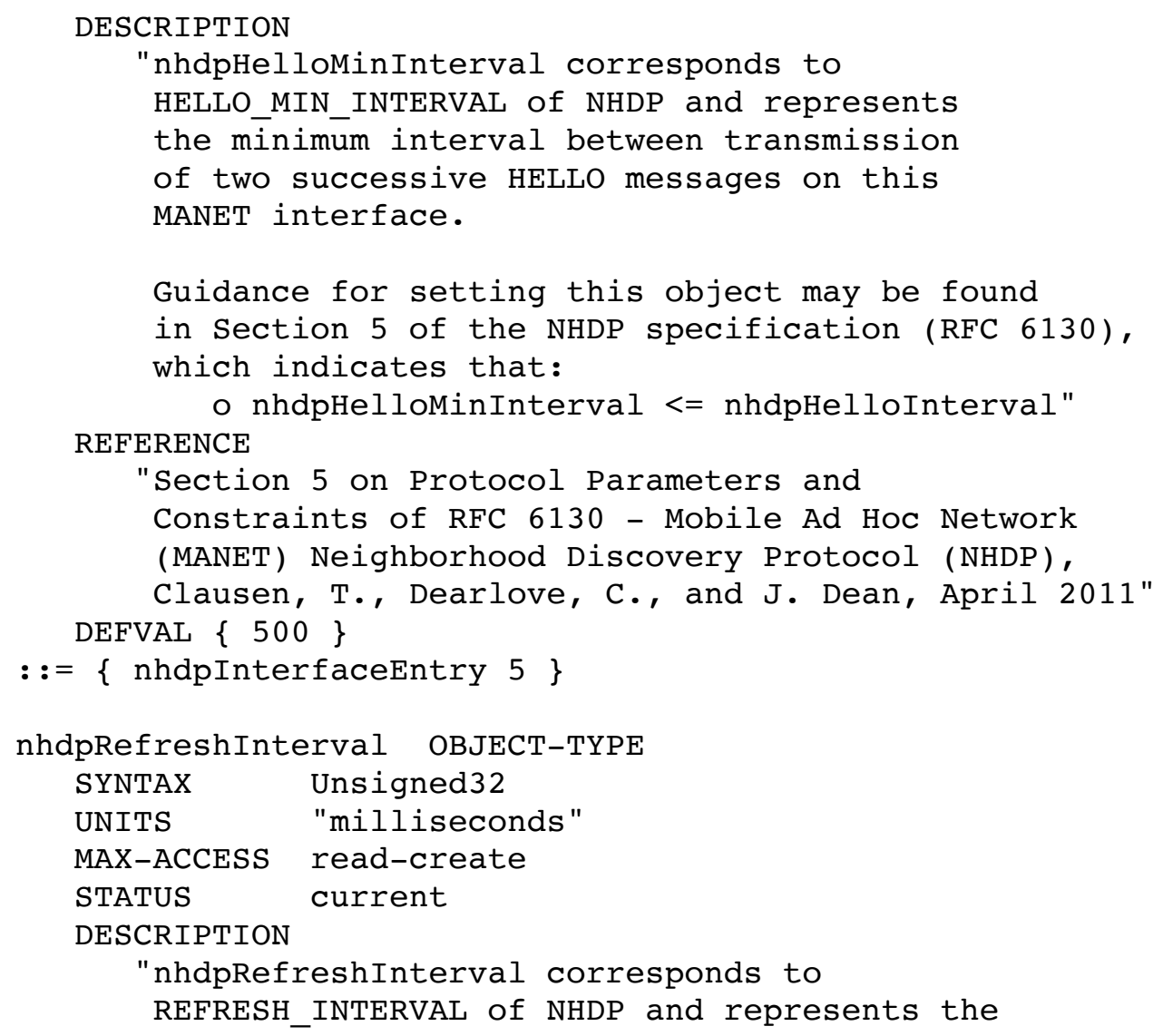


maximum interval between advertisements of each 1-hop neighbor network address and its status. Each advertisement is in a HELLO message on this MANET interface.

Guidance for setting this object may be found in Section 5 of the NHDP specification (RFC 6130), which indicates that: REFERENCE

o nhdpRefreshInterval >= nhdpHelloInterval"

"Section 5 on Protocol Parameters and Constraints of RFC 6130 - Mobile Ad Hoc Network (MANET) Neighborhood Discovery Protocol (NHDP), Clausen, T., Dearlove, C., and J. Dean, April 2011" DEFVAL $\{2000\}$

$::=\{$ nhdpInterfaceEntry 6$\}$

-- Interface Parameters - Information Validity times

--

Herberg, et al.

RFC 7939
Standards Track

The NHDP-MIB
[Page 20]

August 2016

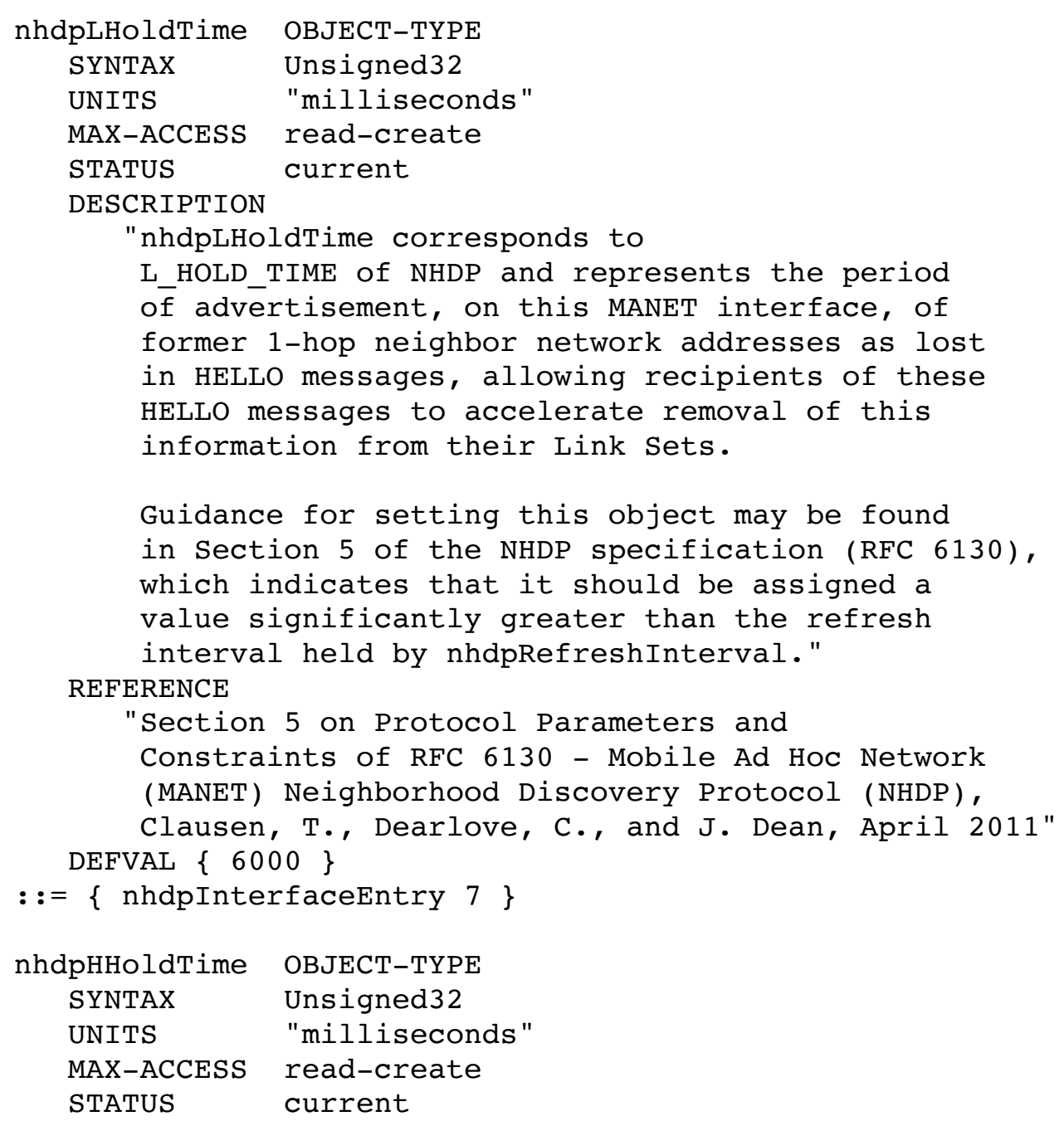




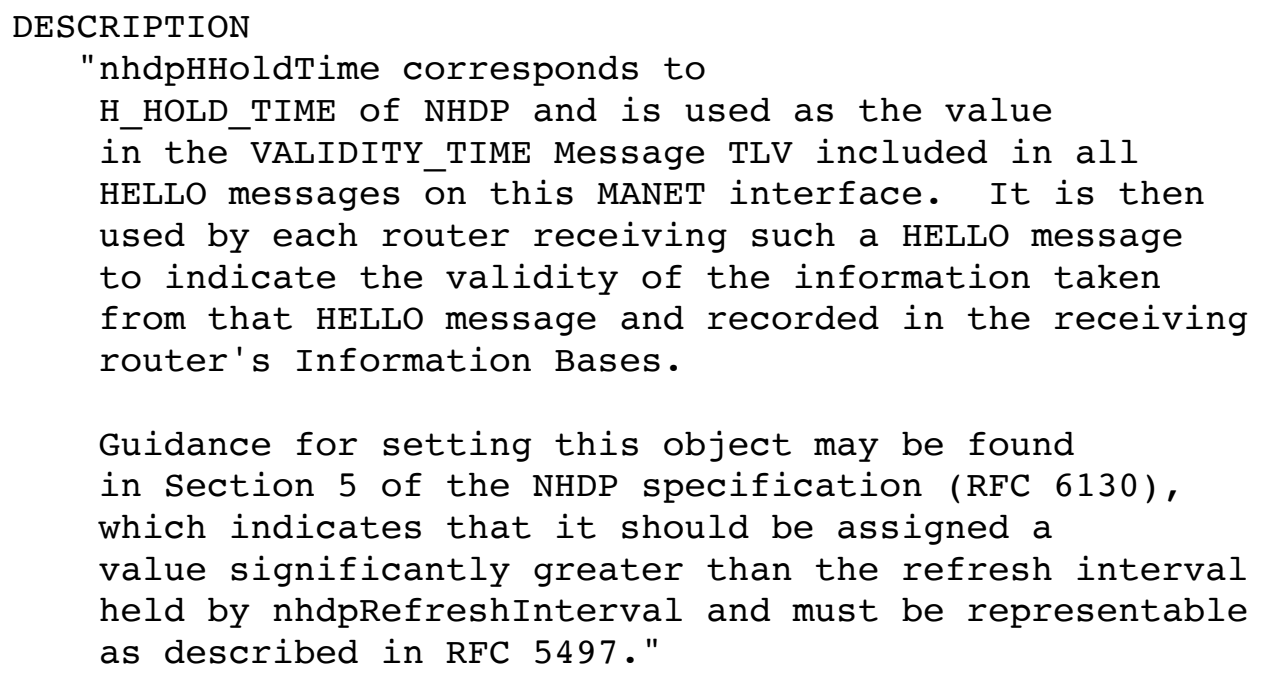

Herberg, et al.

Standards Track

[Page 21]

RFC 7939

The NHDP-MIB

August 2016

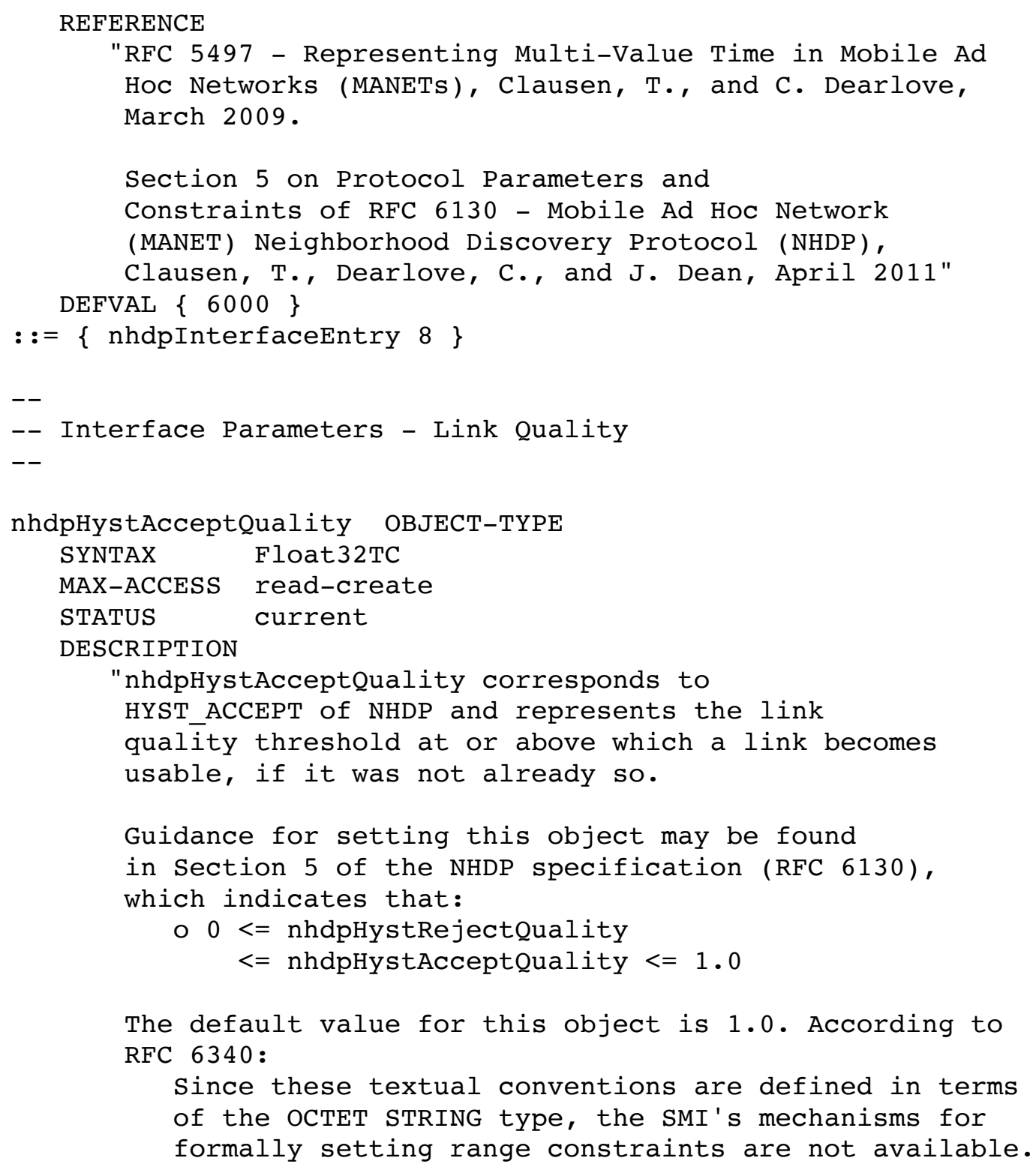


MIB designers using these textual conventions will need to use DESCRIPTION clauses to spell out any applicable range constraints beyond those implied by the underlying IEEE types.

Therefore, this object does not have a DEFVAL clause." REFERENCE

"Section 5 on Protocol Parameters and

Constraints of RFC 6130 - Mobile Ad Hoc Network

(MANET) Neighborhood Discovery Protocol (NHDP),

Clausen, T., Dearlove, C., and J. Dean, April 2011"

DEFVAL $\{1.0\}$ see DESCRIPTION

Herberg, et al.

RFC 7939
Standards Track

The NHDP-MIB
[Page 22]

August 2016

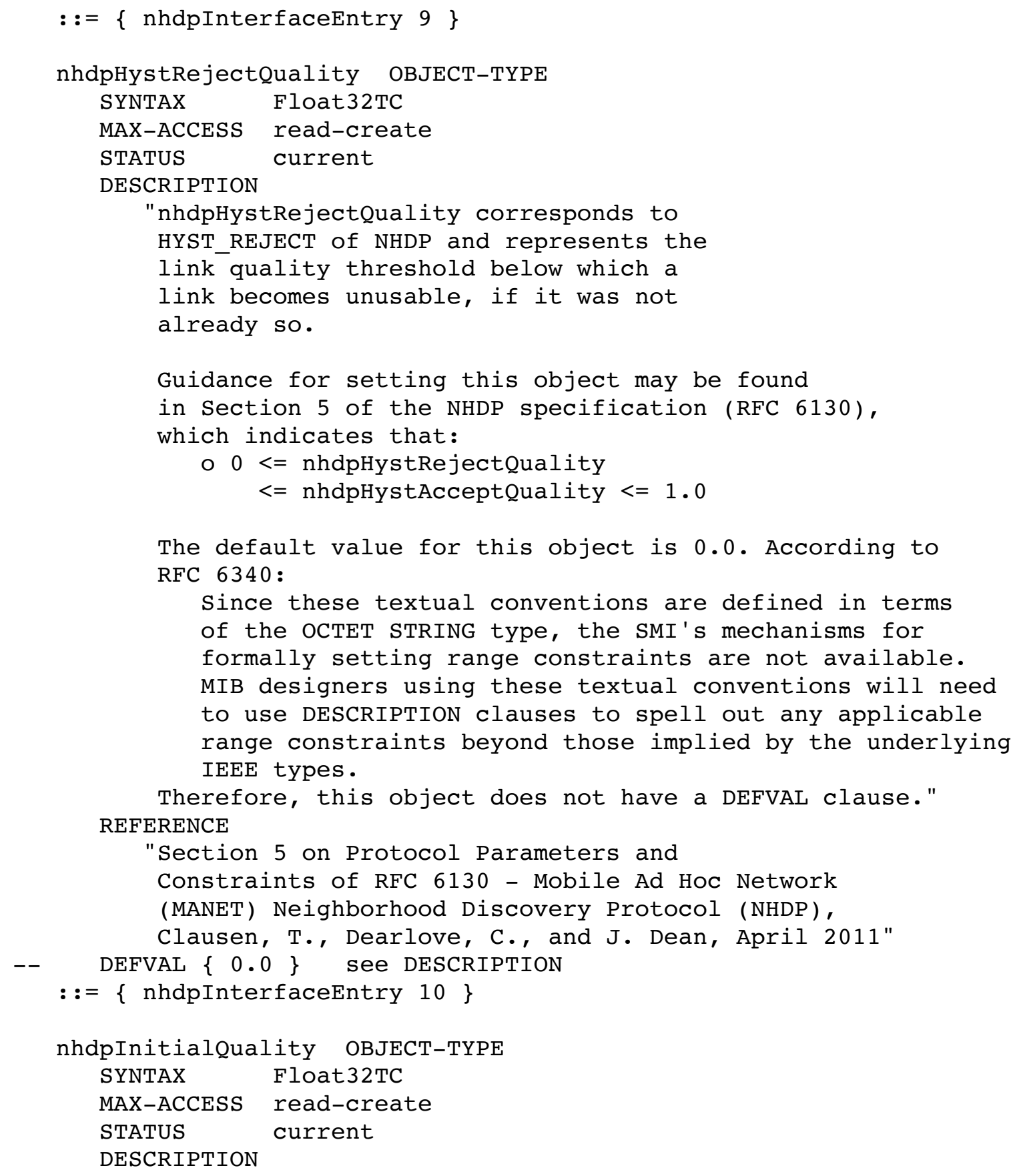


"nhdpInitialQuality corresponds to

INITIAL_QUALITY of NHDP and represents the

initial quality of a newly identified link.

Guidance for setting this object may be found

in Section 5 of the NHDP specification (RFC 6130),

Herberg, et al.

RFC 7939
Standards Track

The NHDP-MIB
[Page 23]

August 2016

which indicates that:

o $0<=$ nhdpInitialQuality $<=1.0$

The default value for this object is 1.0 . According to RFC 6340 :

Since these textual conventions are defined in terms of the OCTET STRING type, the SMI's mechanisms for formally setting range constraints are not available. MIB designers using these textual conventions will need to use DESCRIPTION clauses to spell out any applicable range constraints beyond those implied by the underlying IEEE types.

Therefore, this object does not have a DEFVAL clause." REFERENCE

"Section 5 on Protocol Parameters and

Constraints of RFC 6130 - Mobile Ad Hoc Network

(MANET) Neighborhood Discovery Protocol (NHDP),

Clausen, T., Dearlove, C., and J. Dean, April 2011"

-- DEFVAL $\{1.0\}$ see DESCRIPTION

$::=\{$ nhdpInterfaceEntry 11$\}$

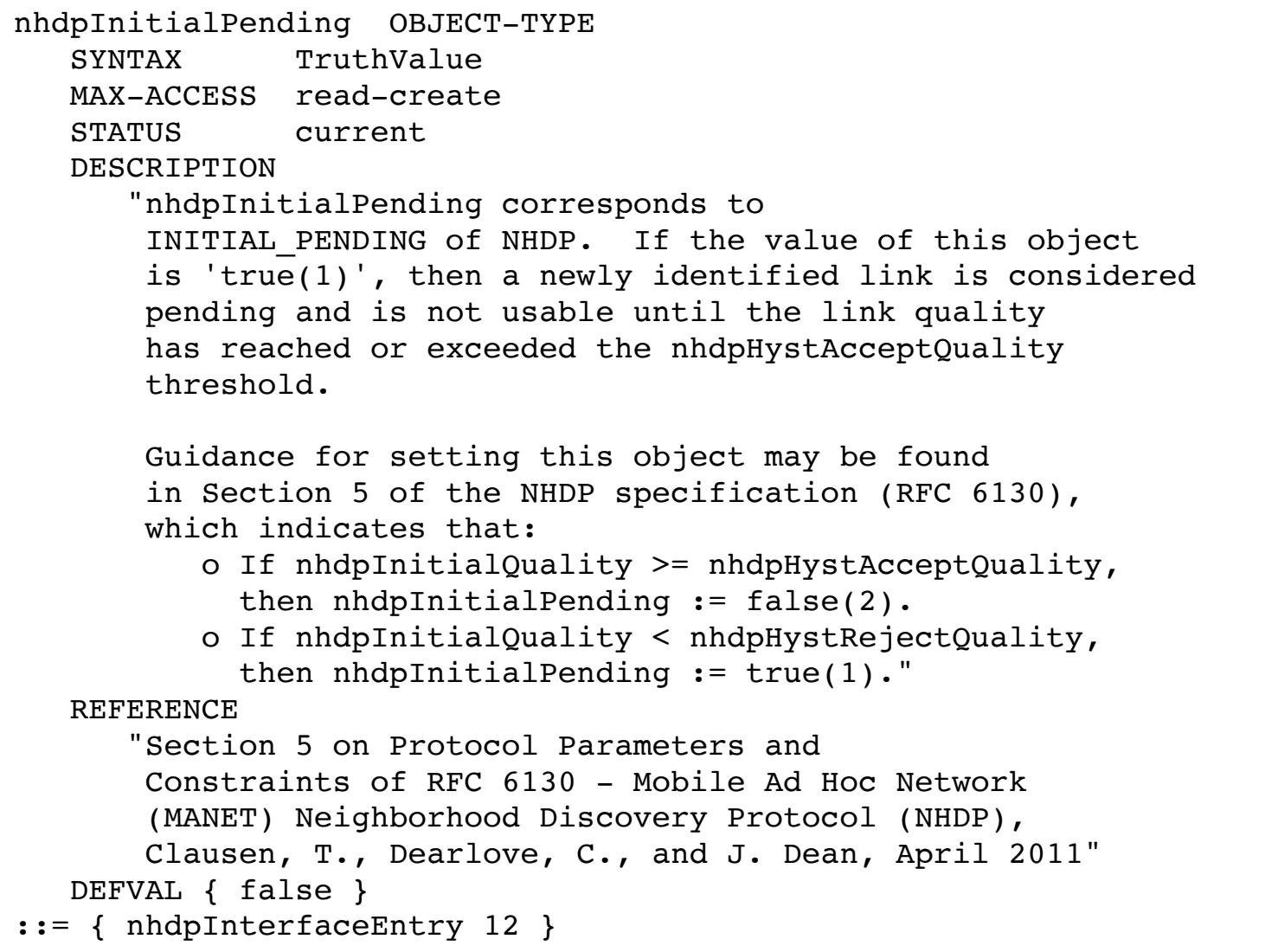


Herberg, et al.

RFC 7939
Standards Track

The NHDP-MIB
[Page 24]

August 2016

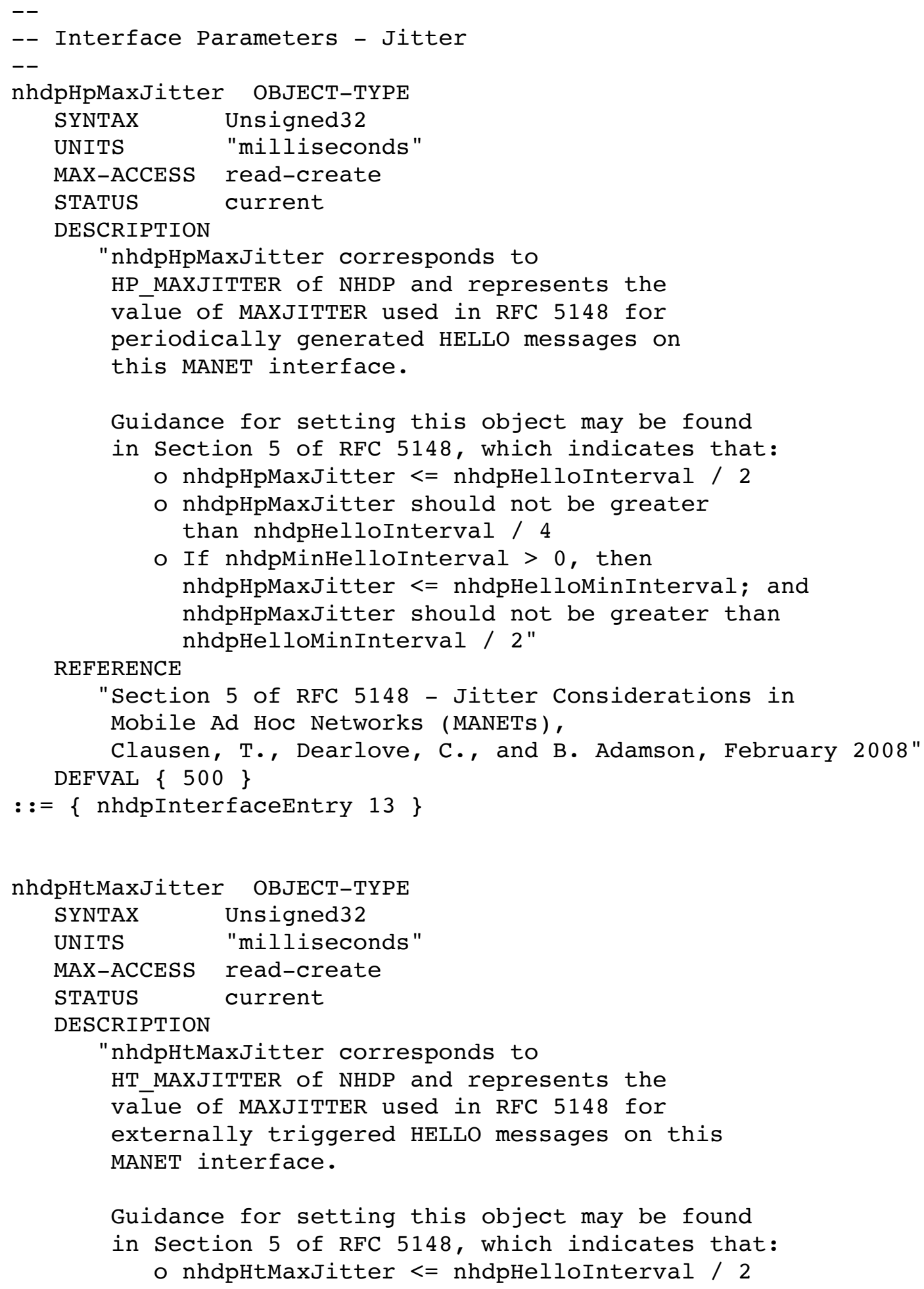


o nhdpHtMaxJitter should not be greater than nhdpHelloInterval / 4

o If nhdpMinHelloInterval >0, then nhdpHtMaxJitter <= nhdpHelloMinInterval; and nhdpHtMaxJitter should not be greater than nhdpHellominInterval / 2"

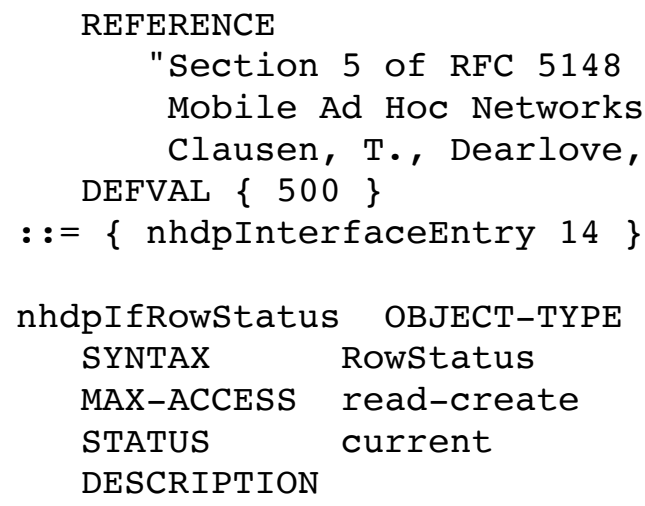




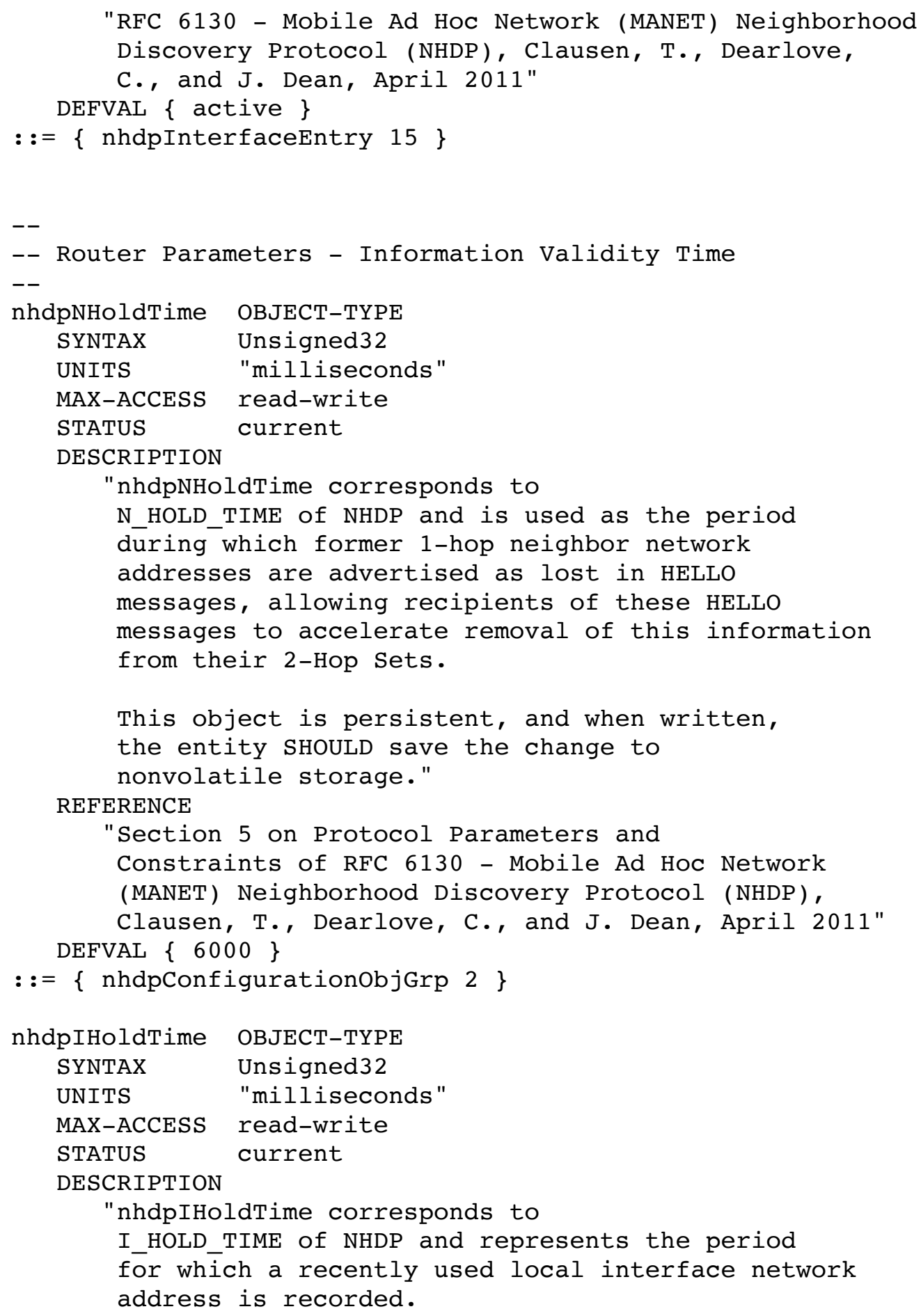

This object is persistent, and when written, the entity SHOULD save the change to nonvolatile storage." 
(MANET) Neighborhood Discovery Protocol (NHDP),

Clausen, T., Dearlove, C., and J. Dean, April 2011" DEFVAL $\{6000\}$

$::=\{$ nhdpConfigurationobjGrp 3$\}$

-- A router's Local Information Base (LIB)

--

-- Local Interface set Table

--

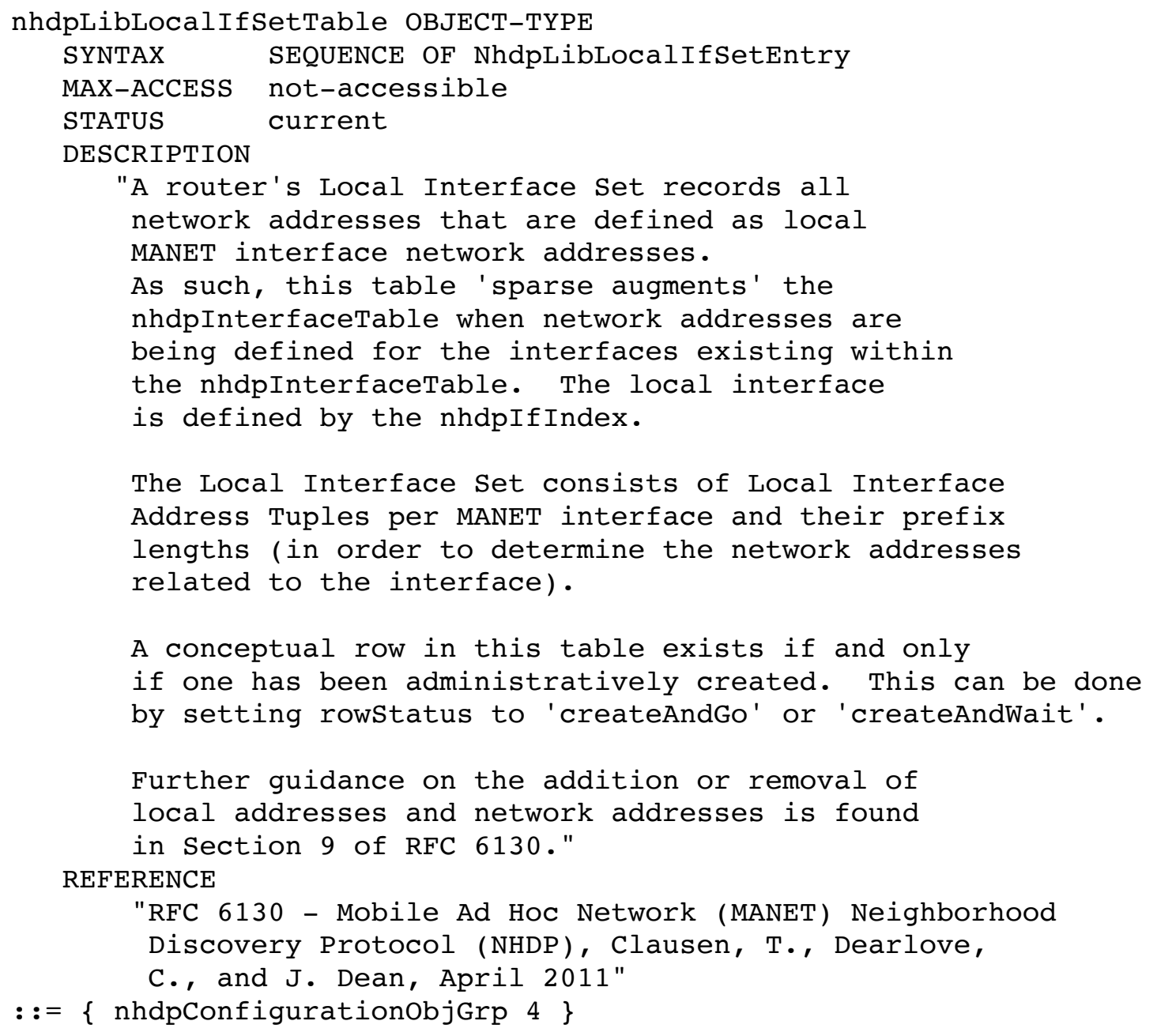

Herberg, et al.

RFC 7939
Standards Track

The NHDP-MIB
[Page 28]

August 2016

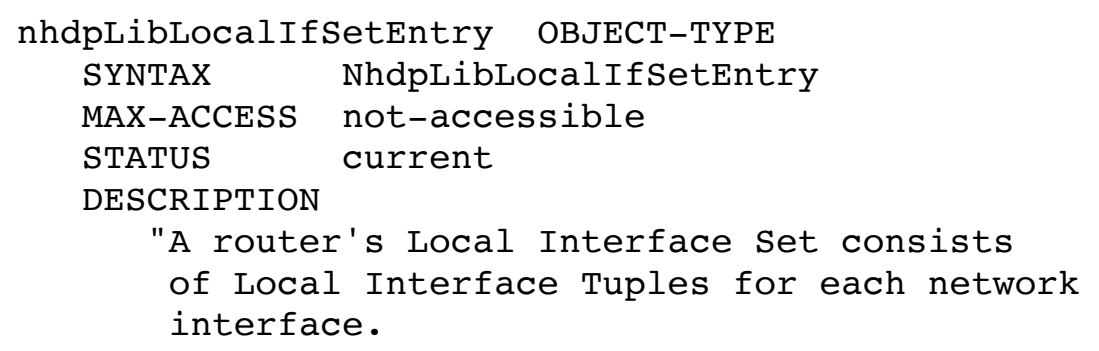

The objects in this table are persistent, and when written, the device SHOULD save the change to 
C., and J. Dean, April 2011"

$::=\{$ nhdpLibLocalifSetEntry 2$\}$

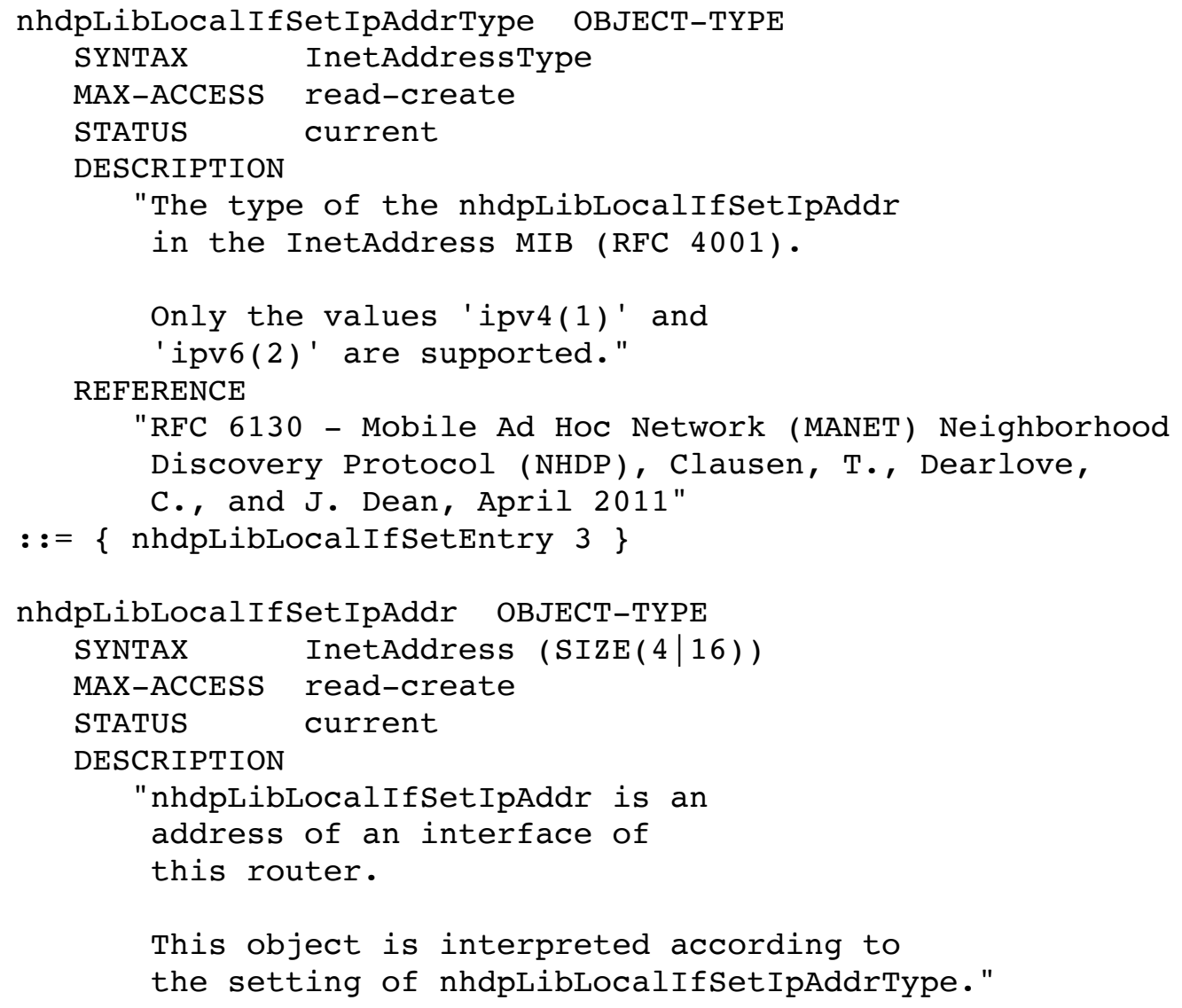

Herberg, et al.

RFC 7939
Standards Track

The NHDP-MIB
[Page 30]

August 2016

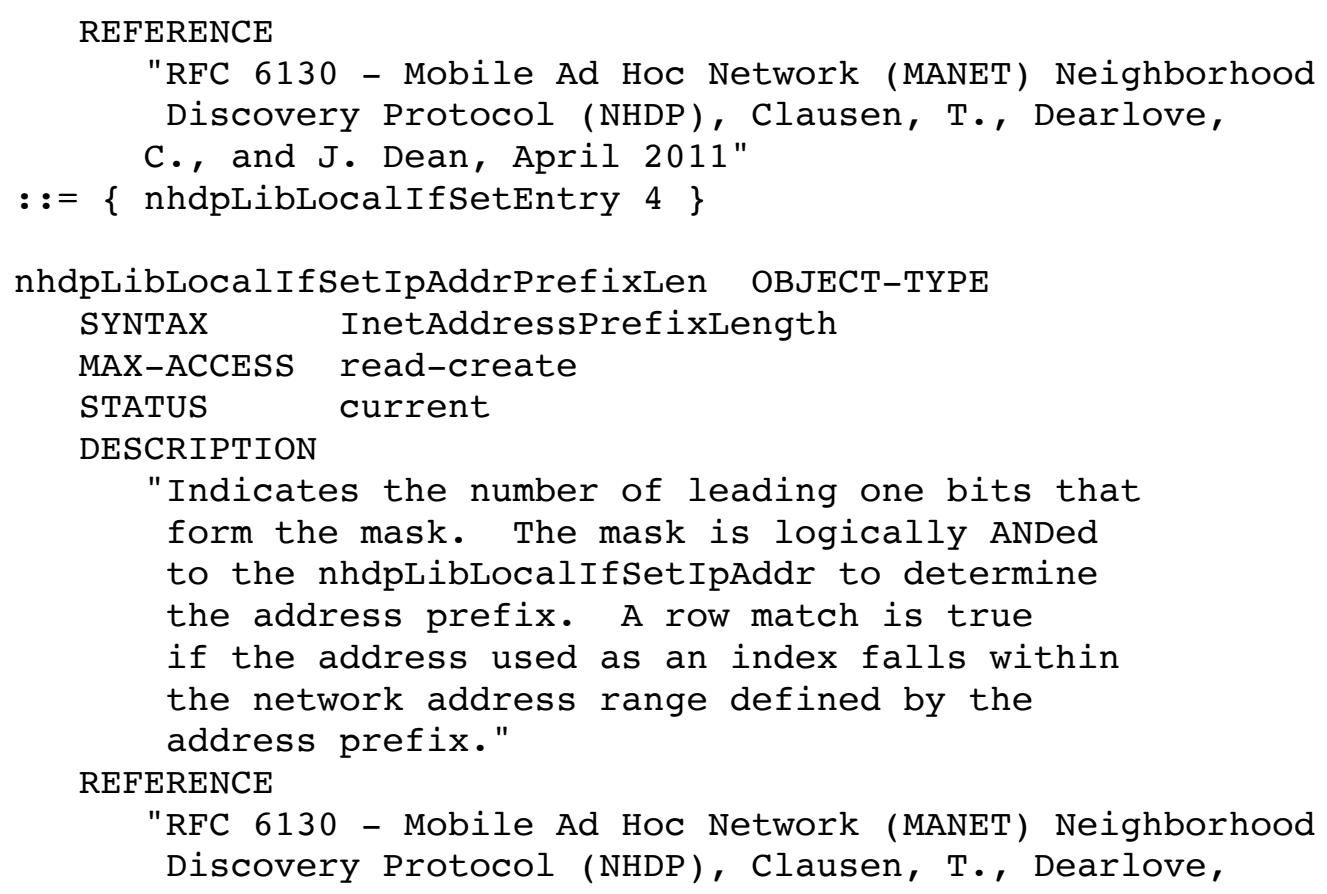


C., and J. Dean, April 2011"

$::=\{$ nhdpLibLocalifSetEntry 5$\}$

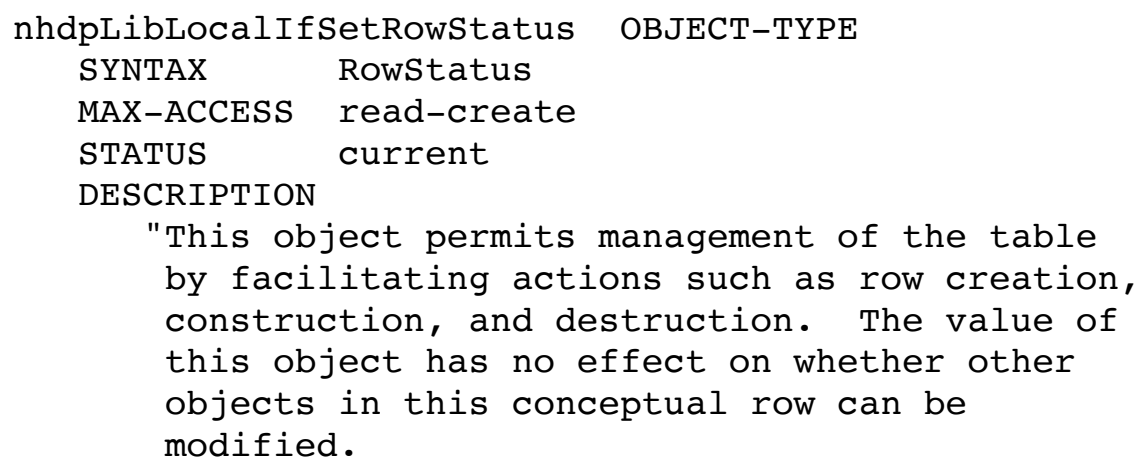

An entry may not exist in the 'active(1)' state unless all read-create objects in the entry have a defined appropriate value. As no objects in this table have DEFVAL clauses, the management station MUST specify the values of all read-create objects prior to this row transitioning to the 'active(1)' state.

When this object transitions to 'active(1)', all objects in this row SHOULD be written to nonvolatile (stable) storage. Read-create objects in this row MAY be modified. When an object in a row with nhdpIfRowStatus of 'active(1)' is changed, then the updated value MUST be reflected in NHDP,

Herberg, et al.

RFC 7939
Standards Track

The NHDP-MIB
[Page 31]

August 2016
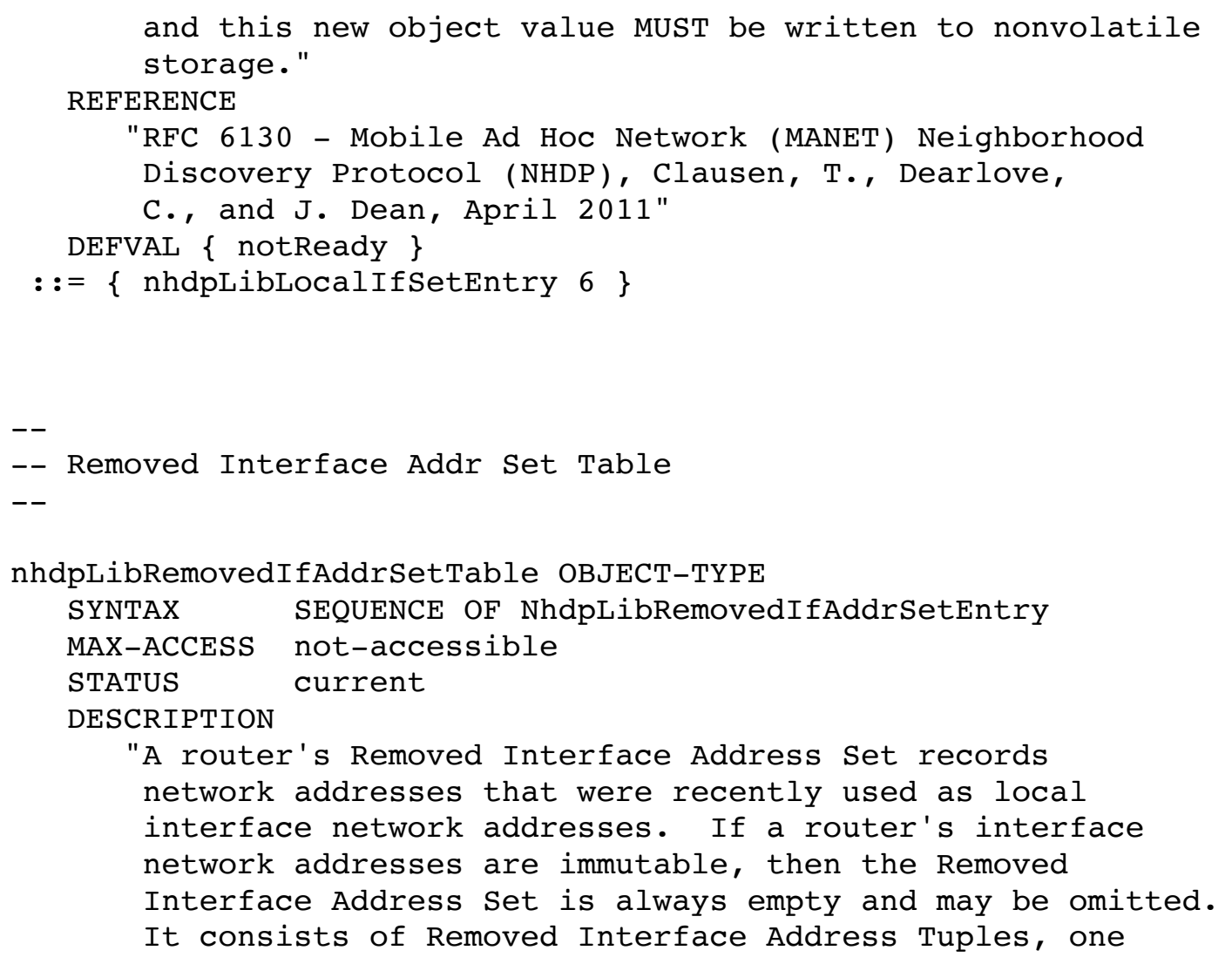


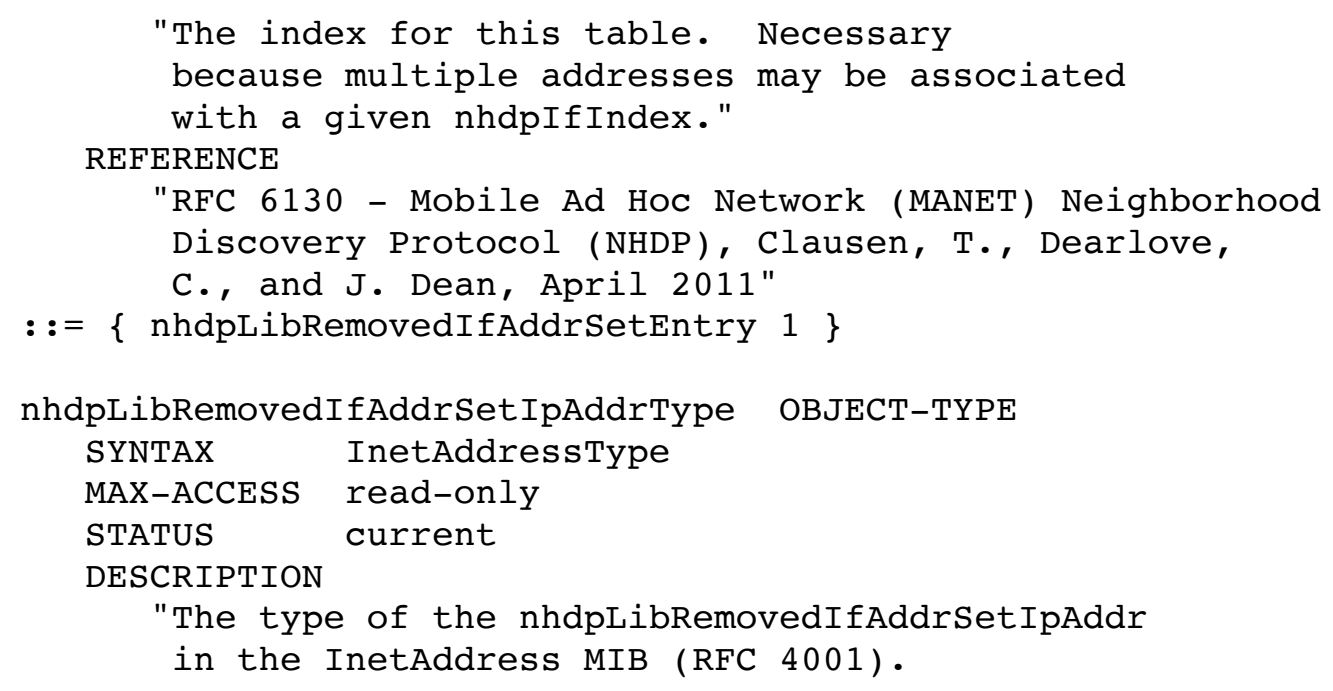

Herberg, et al.

Standards Track

[Page 33]

RFC 7939

The NHDP-MIB

August 2016

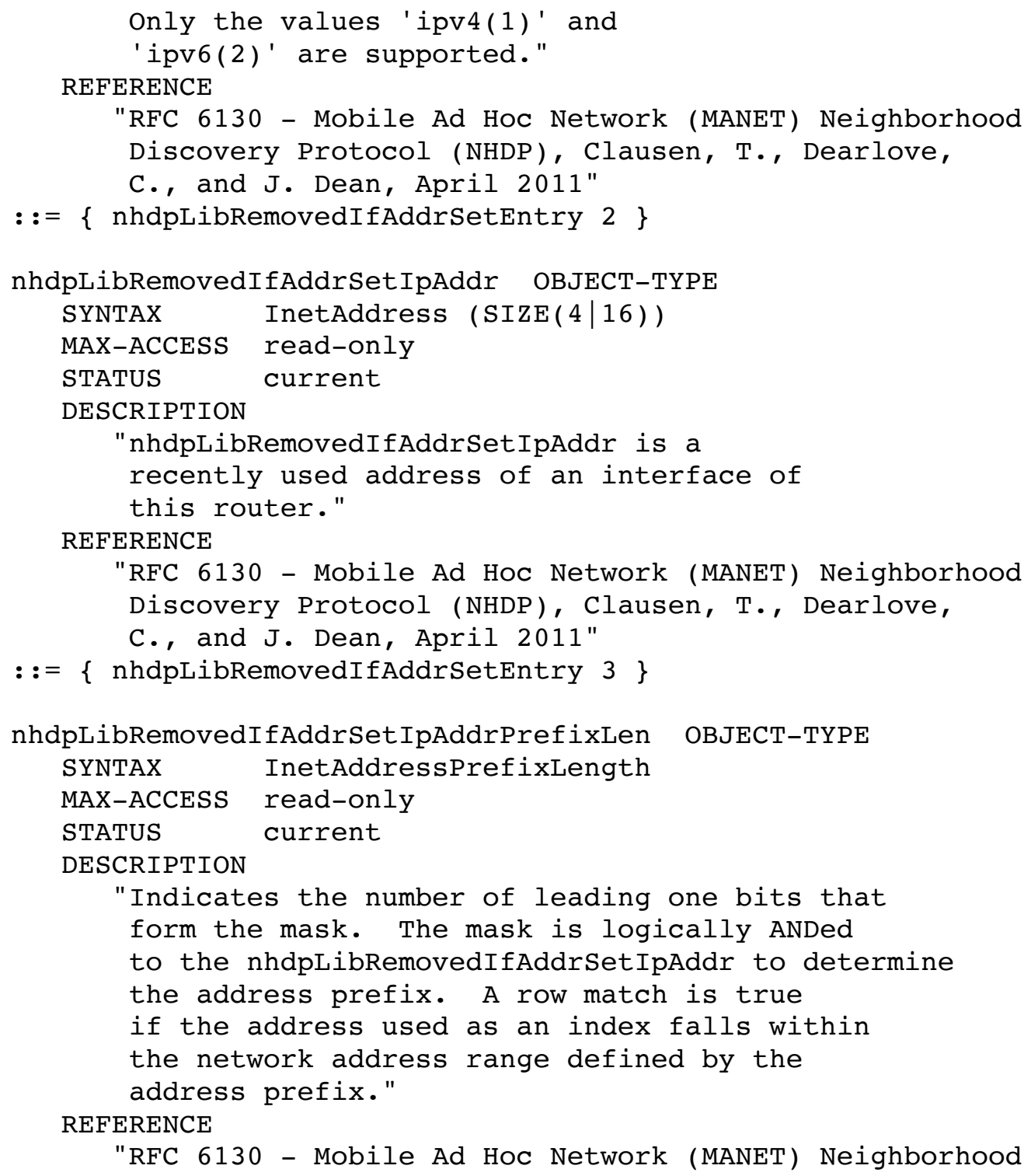


Discovery Protocol (NHDP), Clausen, T., Dearlove,

C., and J. Dean, April 2011"

$::=\{$ nhdpLibRemovedIfAddrSetEntry 4$\}$

nhdpLibRemovedIfAddrSetIfIndex OBJECT-TYPE

SYNTAX InterfaceIndex

MAX-ACCESS read-only

STATUS current

DESCRIPTION

"Specifies the local IfIndex from which this

IP address was recently removed."

Herberg, et al.

RFC 7939

REFERENCE

"RFC 6130 - Mobile Ad Hoc Network (MANET) Neighborhood

Discovery Protocol (NHDP), Clausen, T., Dearlove,

C., and J. Dean, April 2011"

$::=\{$ nhdpLibRemovedIfAddrSetEntry 5$\}$

nhdpLibRemovedIfAddrSetIRTime OBJECT-TYPE

SYNTAX Timestamp

MAX-ACCESS read-only

STATUS current

DESCRIPTION

"nhdpLibRemovedIfAddrSetIRTime specifies the value of sysUpTime when this entry should expire and be removed from the nhdpLibRemovedIfAddrSetTable."

REFERENCE

"RFC 6130 - Mobile Ad Hoc Network (MANET) Neighborhood

Discovery Protocol (NHDP), Clausen, T., Dearlove,

C., and J. Dean, April 2011"

$::=\{$ nhdpLibRemovedIfAddrSetEntry 6$\}$

-- nhdpStateobjGrp

-- Contains information describing the current state of the NHDP

-- process on this router.

nhdpstateobjGrp OBJECT IDENTIFIER ::= \{ nhdpobjects 2$\}$

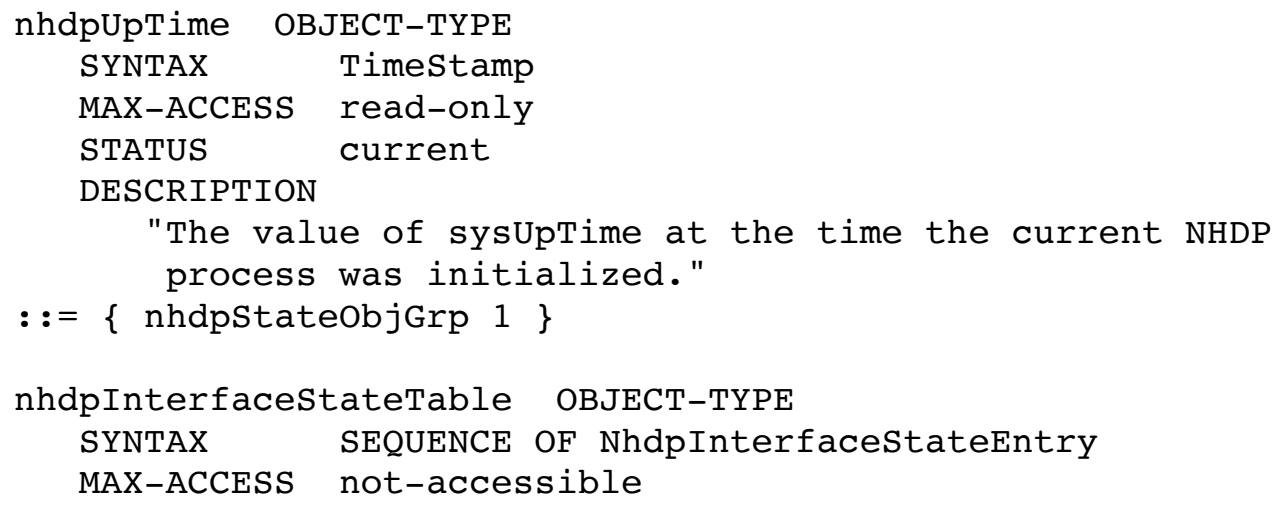




\author{
STATUS current \\ DESCRIPTION \\ "nhdpInterfacestateTable lists state information \\ related to specific interfaces of this router. \\ The value of nhdpIfIndex is an ifIndex from the \\ Interfaces Group defined in the Interfaces Group \\ MIB.
}

Herberg, et al. Standards Track

[Page 35]

RFC 7939

The NHDP-MIB

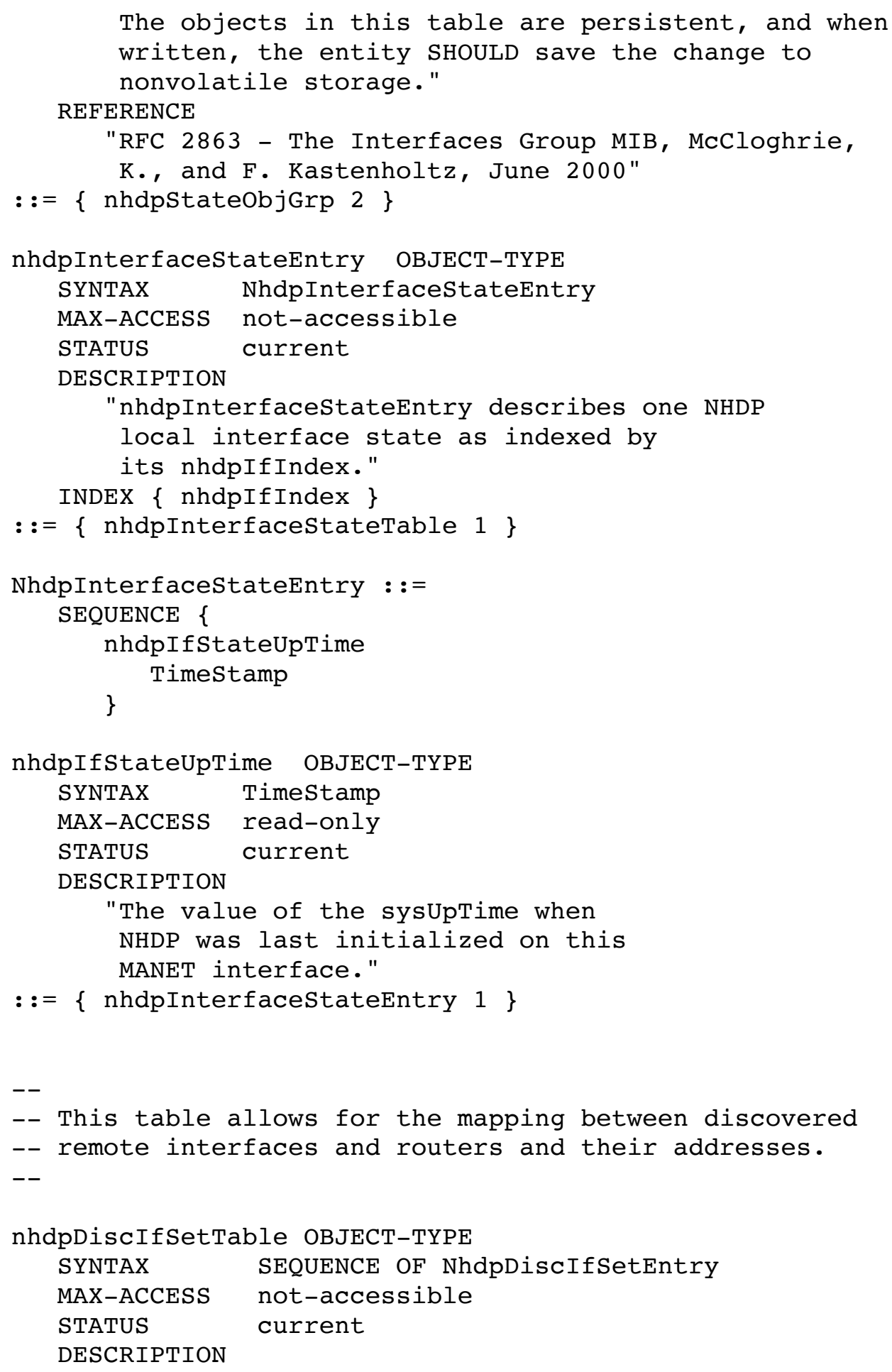




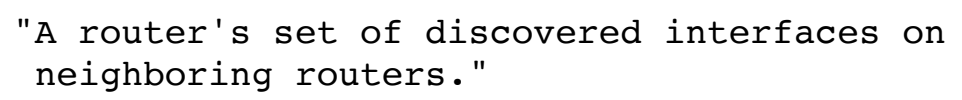

Herberg, et al.

RFC 7939
Standards Track

The NHDP-MIB
[Page 36]

August 2016

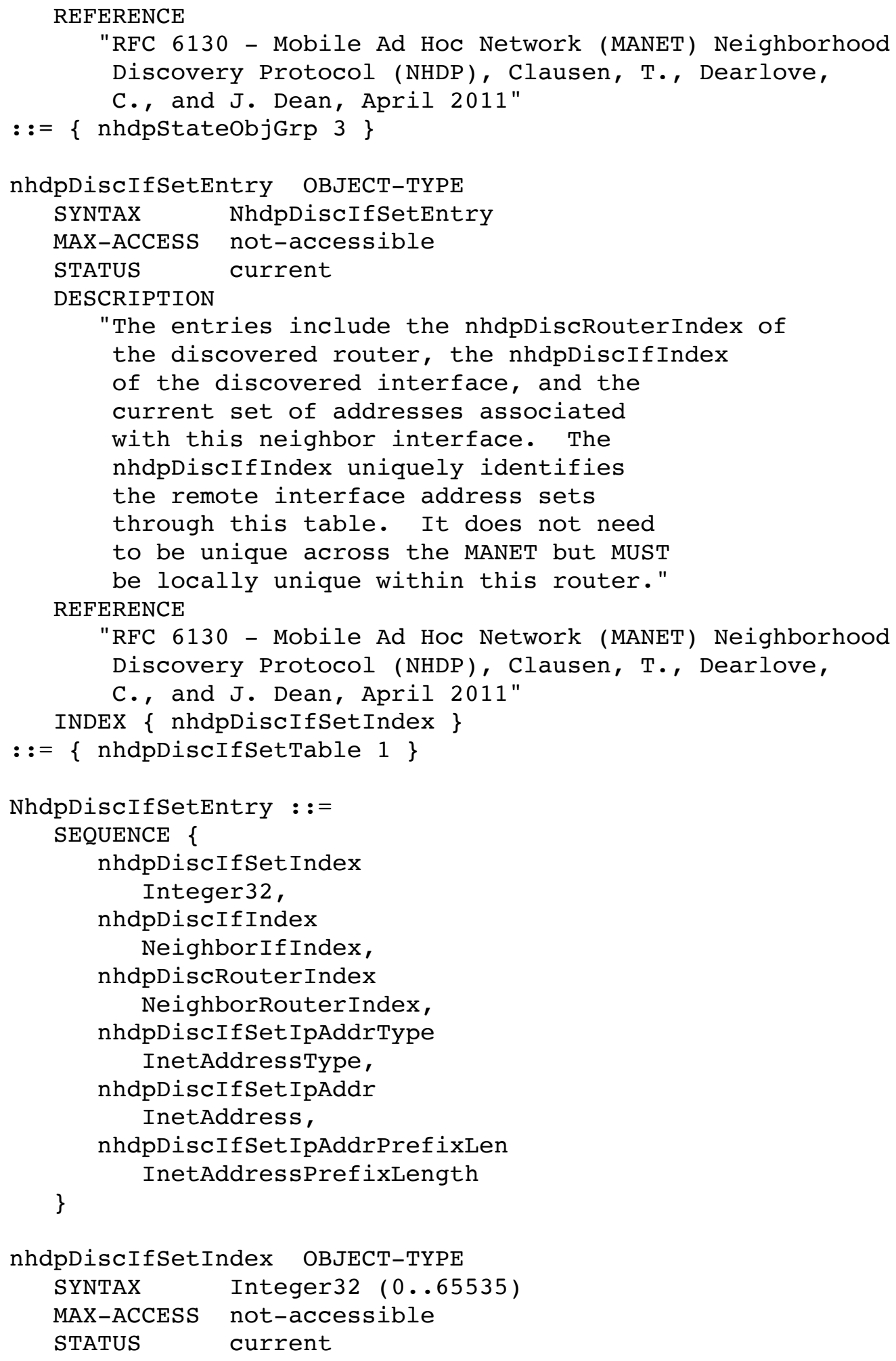


Herberg, et al.

RFC 7939
Standards Track

The NHDP-MIB
[Page 37]

August 2016

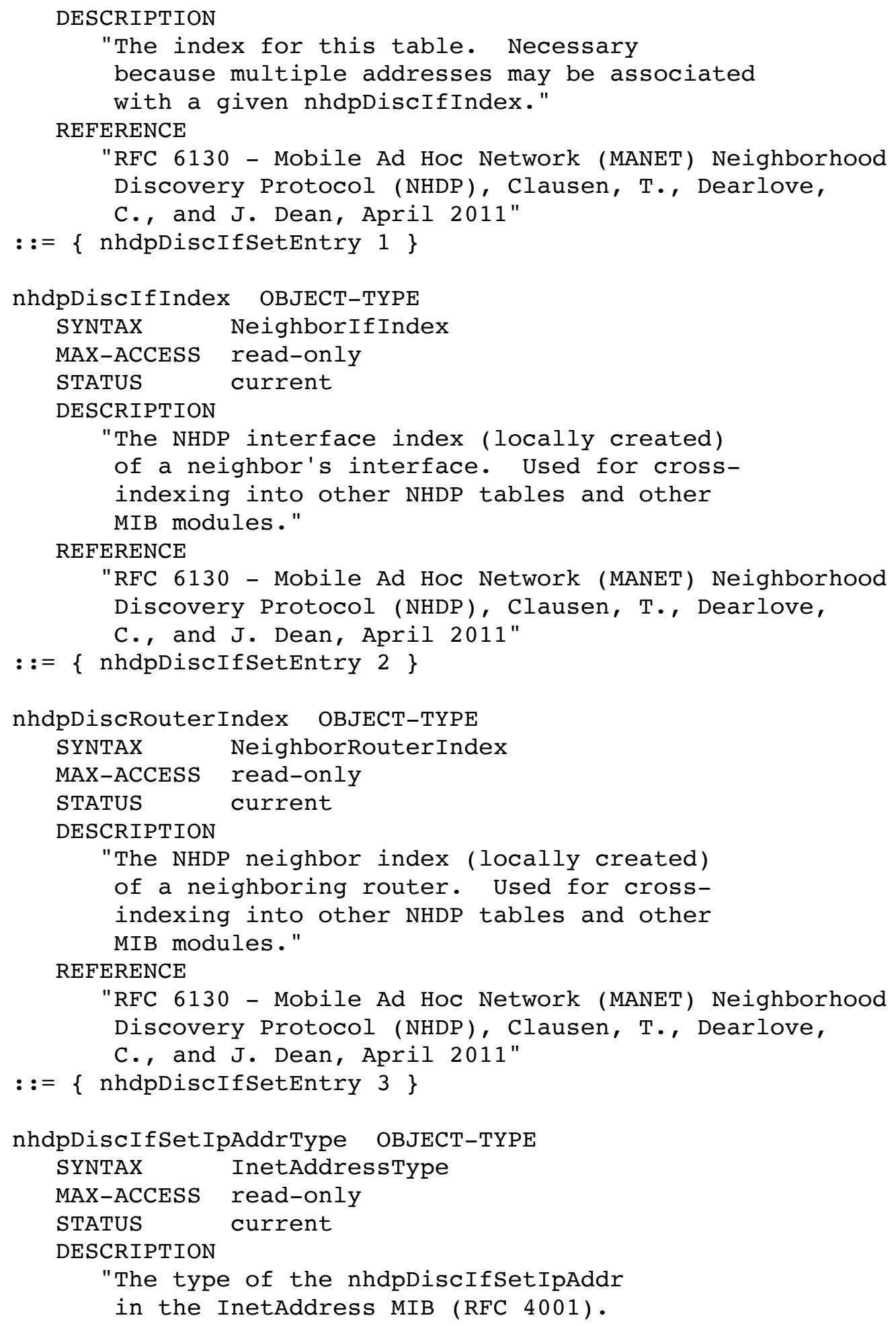




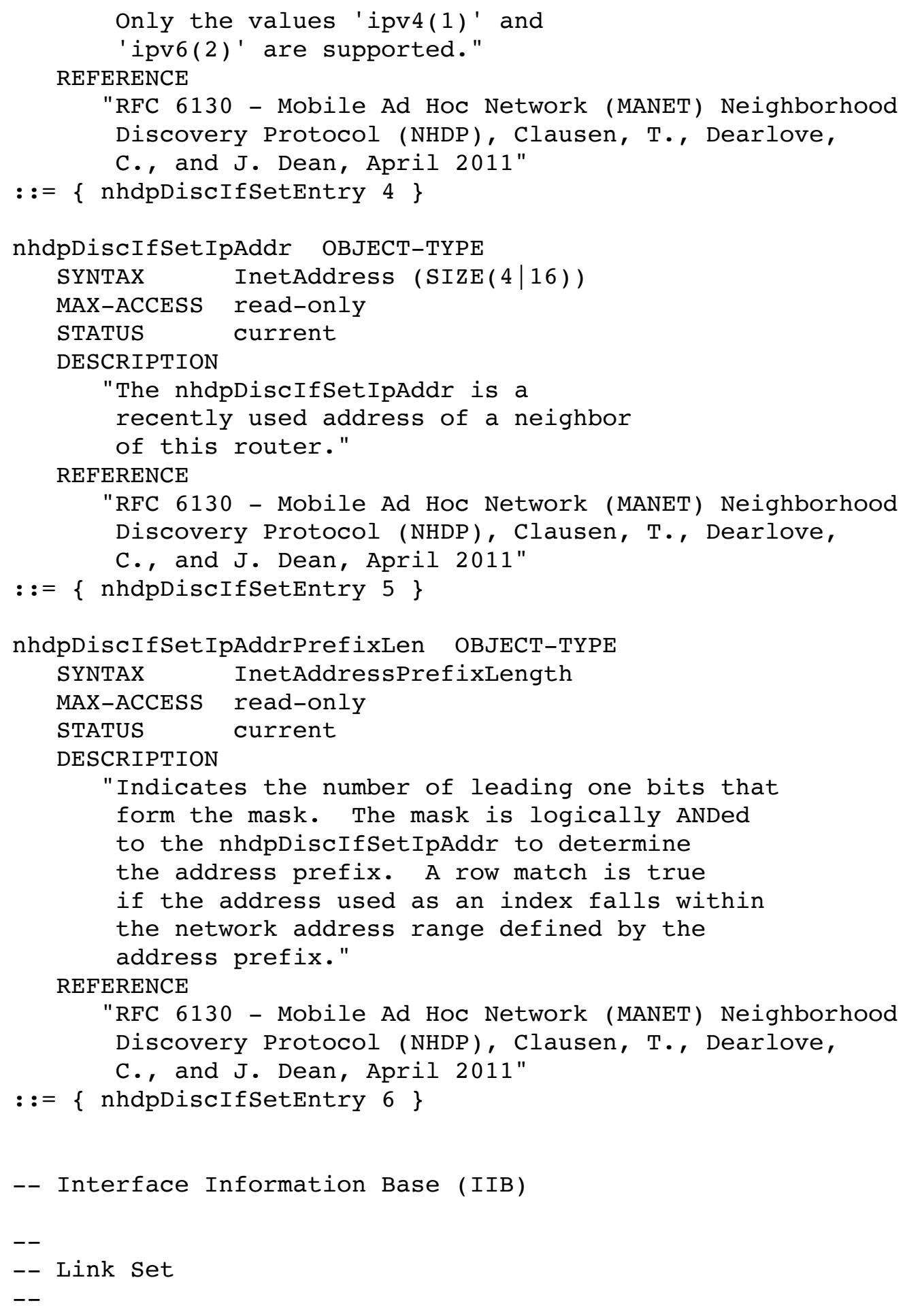

Herberg, et al.

RFC 7939
Standards Track

The NHDP-MIB
[Page 39]

August 2016

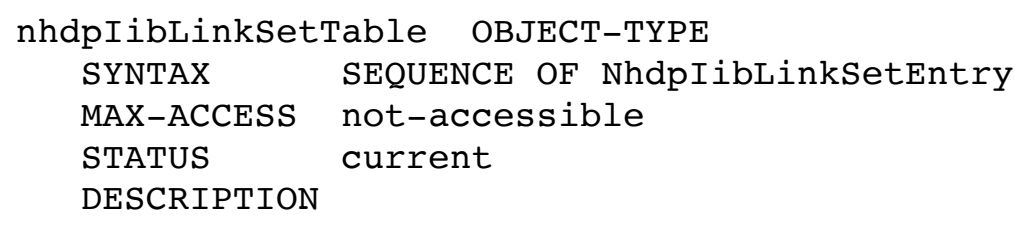




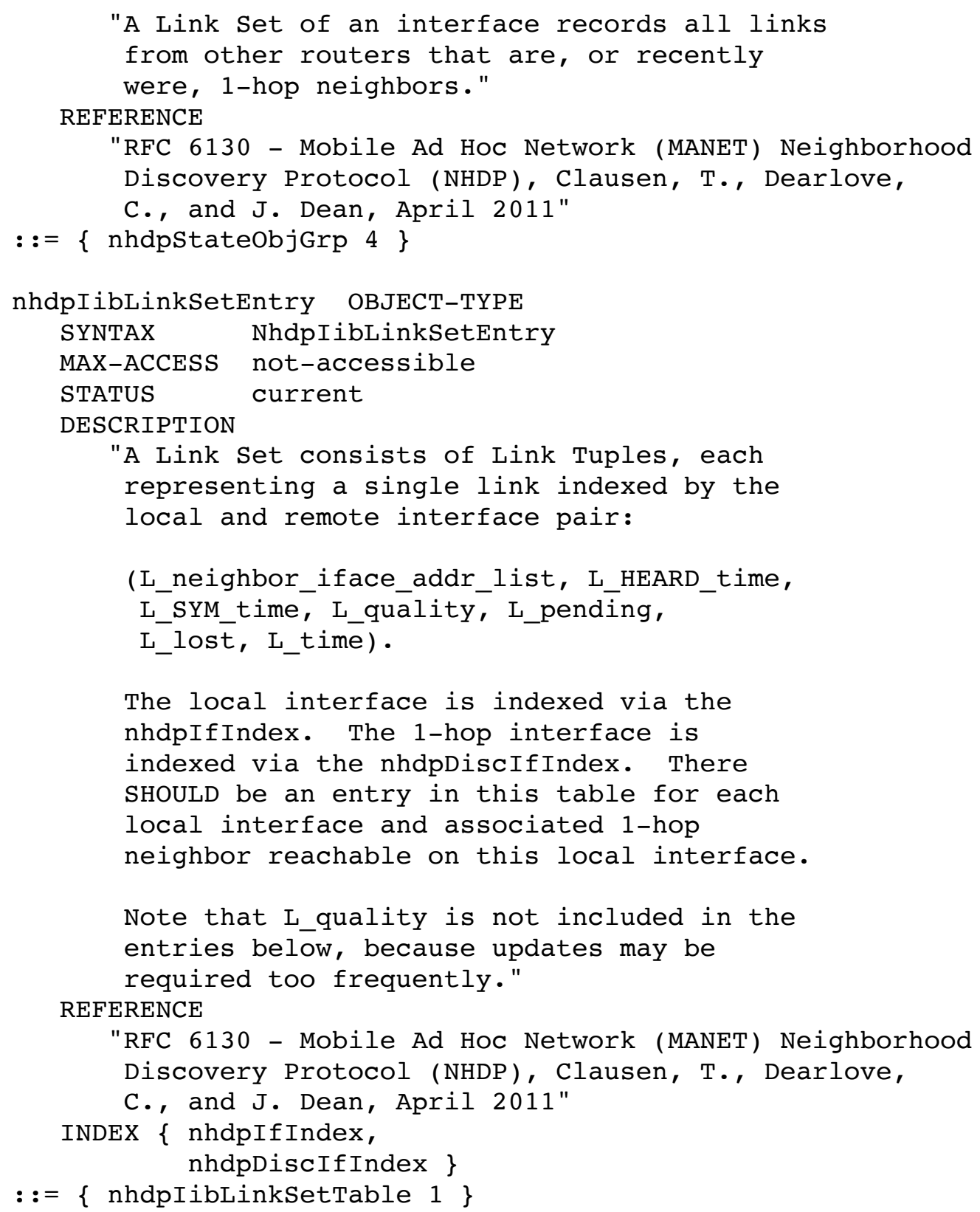

Herberg, et al.

RFC 7939
Standards Track

The NHDP-MIB
[Page 40]

August 2016

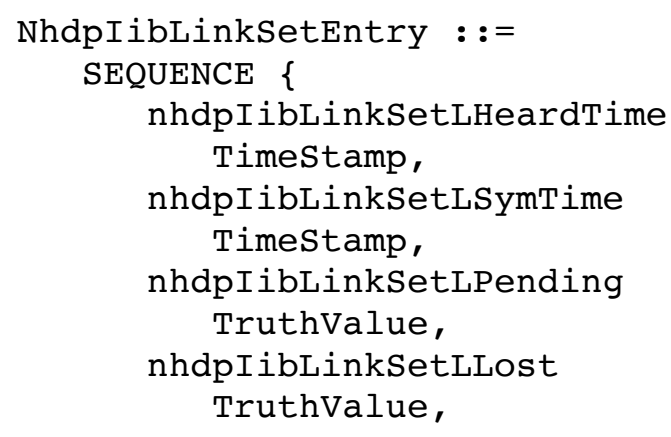




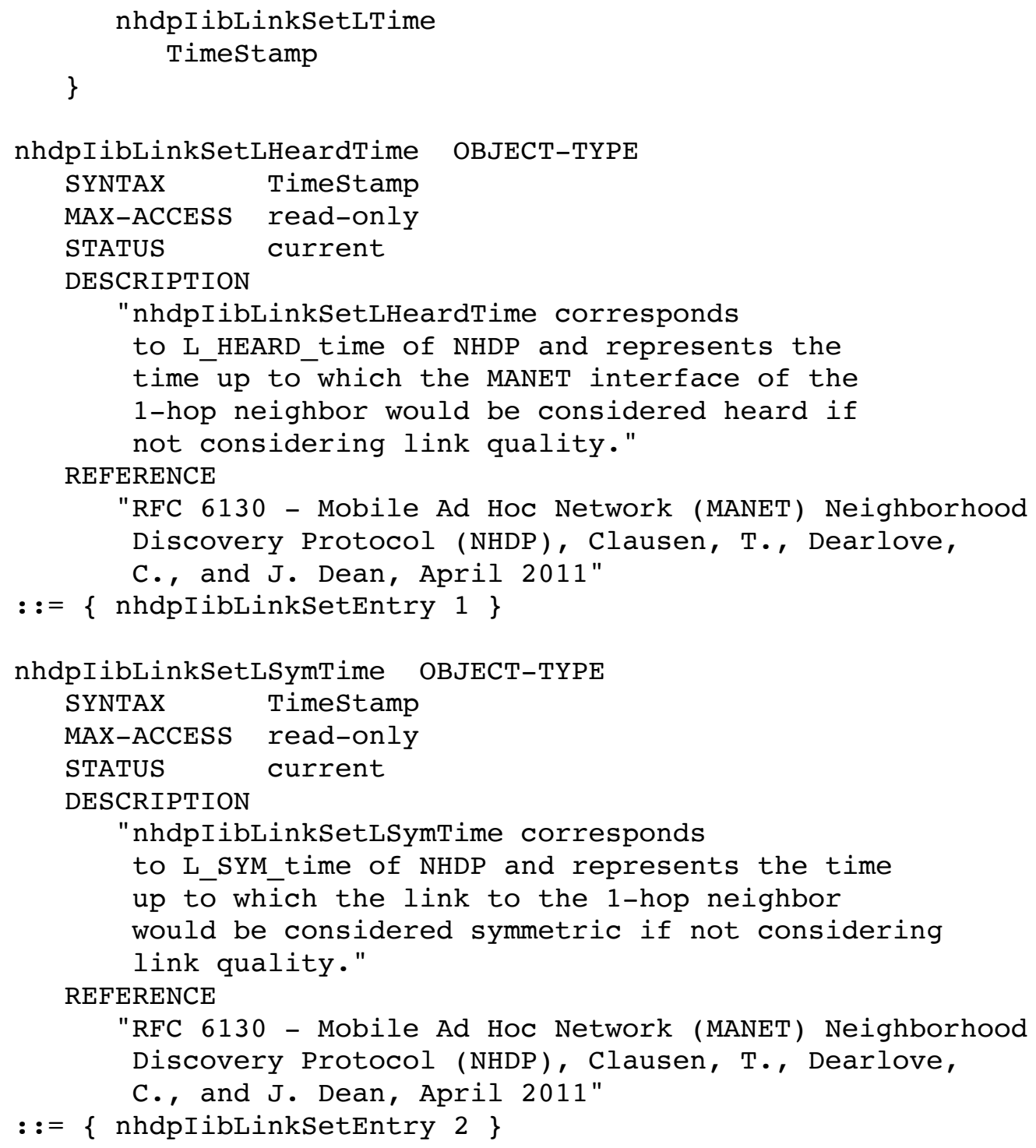

Herberg, et al.

RFC 7939
Standards Track

The NHDP-MIB
[Page 41]

August 2016

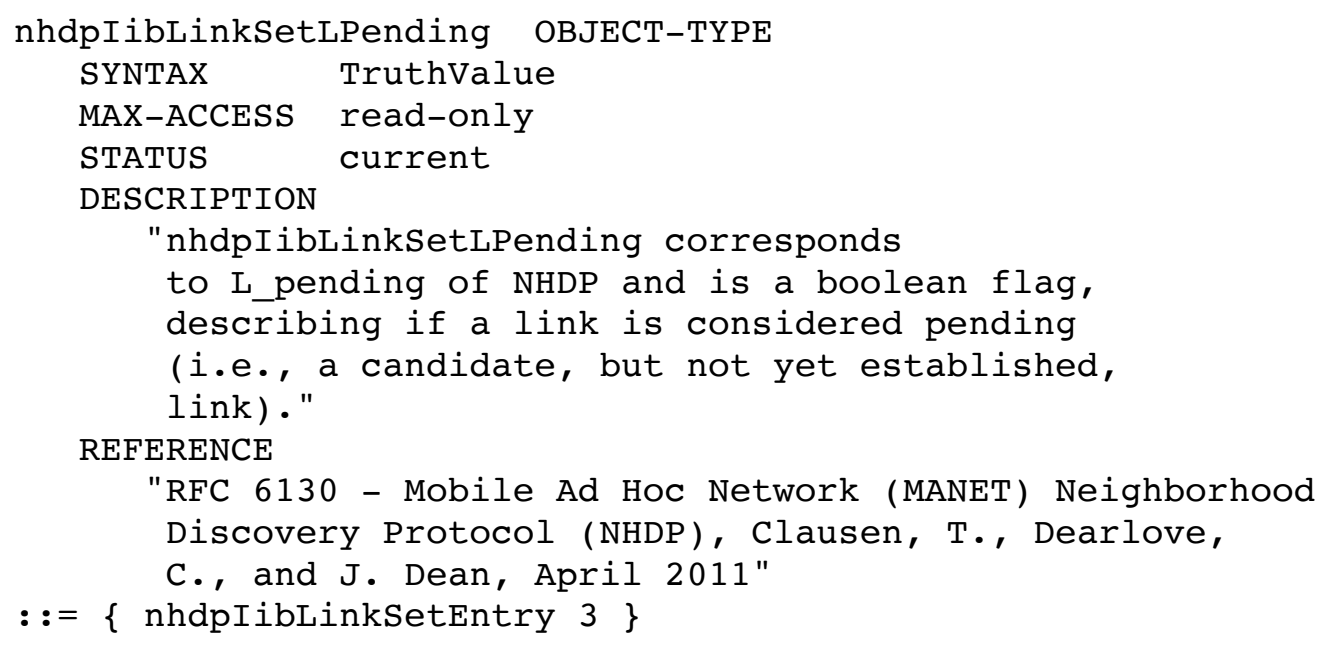



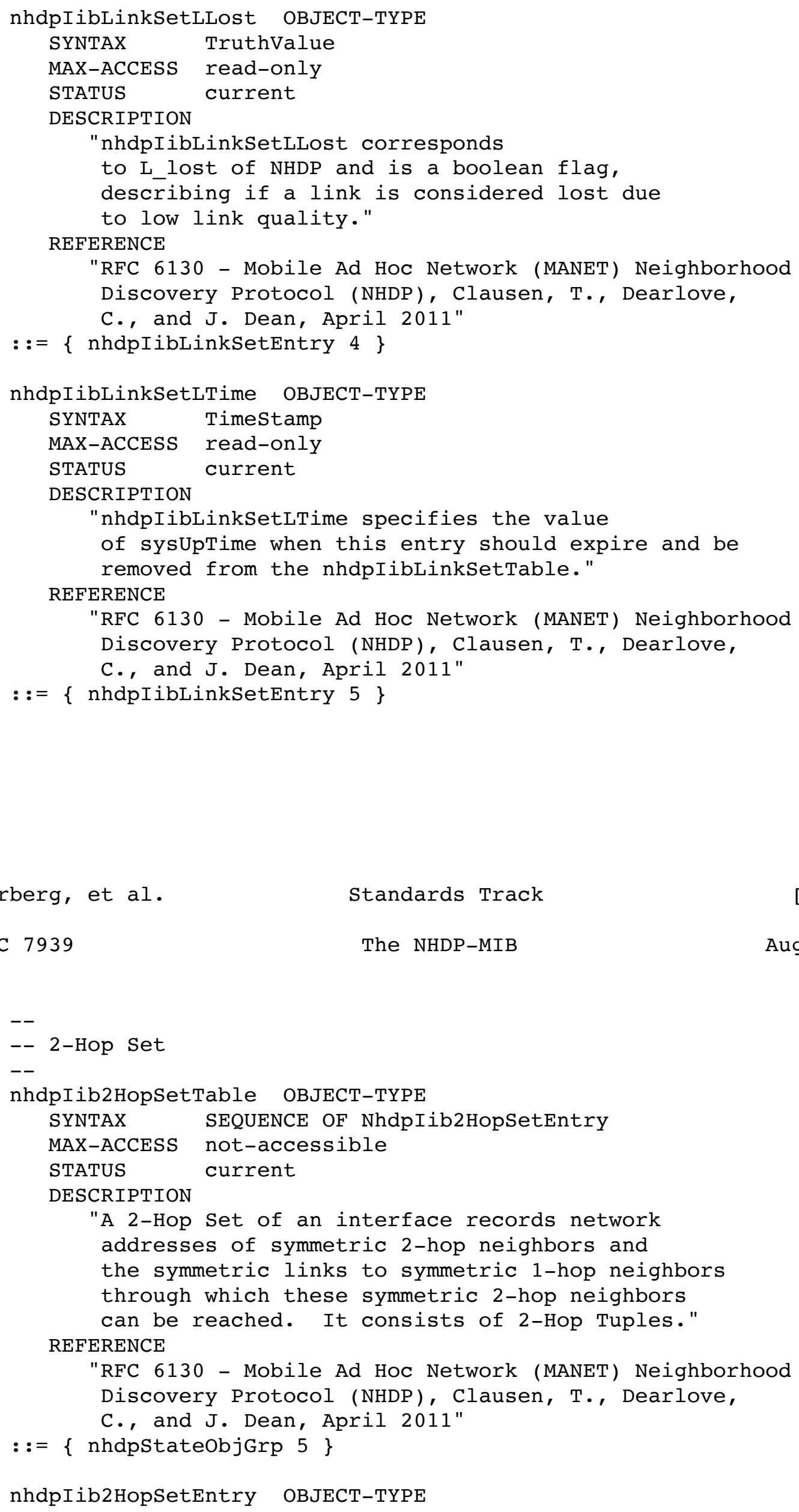


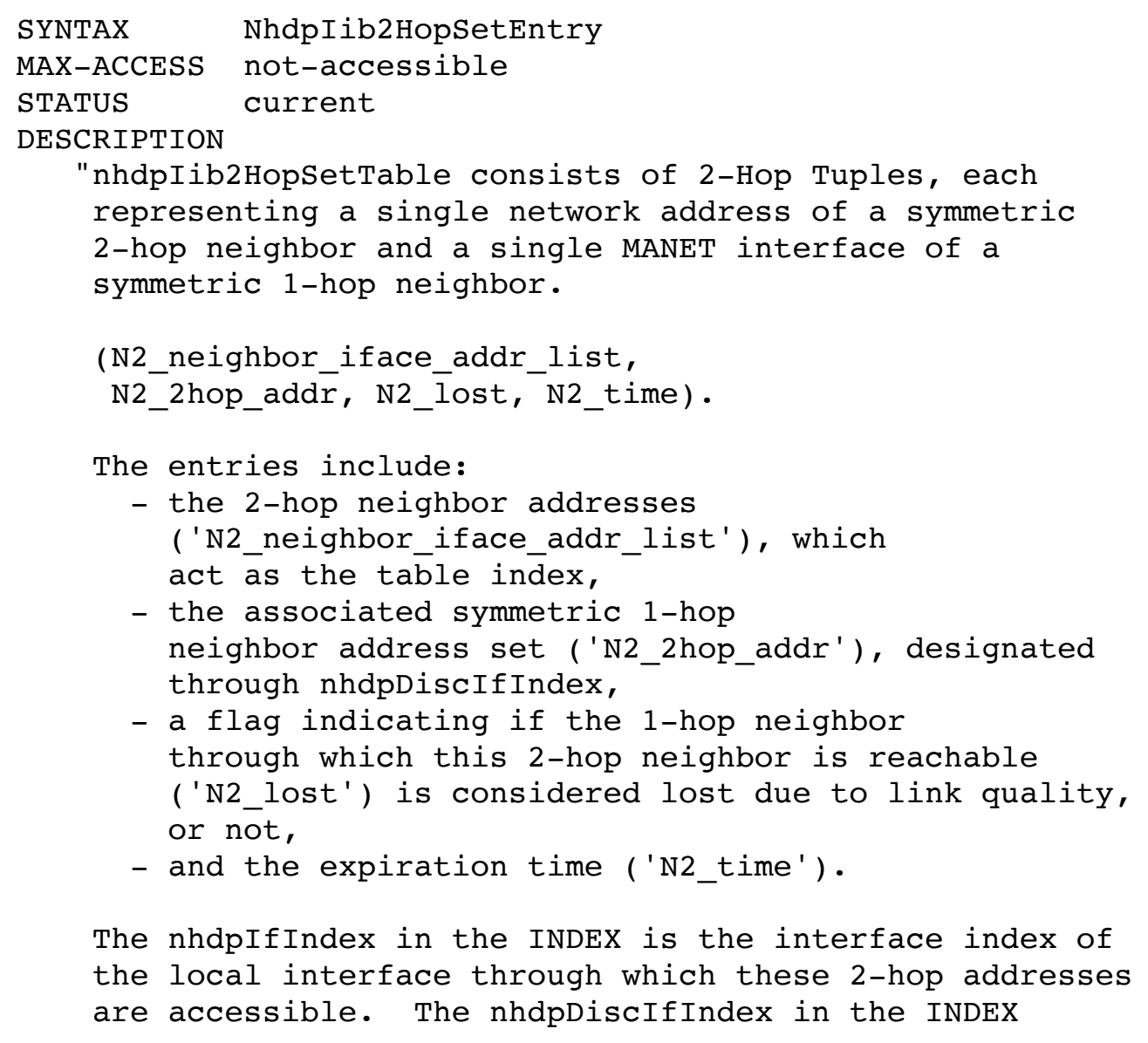

Herberg, et al.

RFC 7939
Standards Track

The NHDP-MIB
[Page 43]

August 2016

represents the 1-hop neighbor interface through which

these 2-hop neighbor addresses are reachable."

\section{REFERENCE}

"RFC 6130 - Mobile Ad Hoc Network (MANET) Neighborhood

Discovery Protocol (NHDP), Clausen, T., Dearlove,

C., and J. Dean, April 2011

and

RFC 7466 - An Optimization for the Mobile Ad Hoc

Network (MANET) Neighborhood Discovery Protocol (NHDP),

Dearlove, C., and T. Clausen, March 2015"

INDEX \{ nhdpIfIndex, nhdpDiscIfIndex, nhdpIib2 HopSet IpAddress Type,

\} nhdpIib2HopSet IpAddress

$::=\{$ nhdpIib2HopsetTable 1$\}$

NhdpIib2HopSetEntry : : = SEQUENCE \{

nhdpI ib2 HopSet IpAddress Type

InetAddressType,

nhdpI ib2HopSet IpAddress

InetAddress,

nhdpIib2HopSetIpAddrPrefixLen 
InetAddressPrefixLength,

nhdpI ib2 HopSet 1 Hop I f Index

NeighborIfIndex,

nhdpIib2 HopSetN2Time

Timestamp,

nhdpI ib2 HopSetN2Lost

Truthvalue

\}

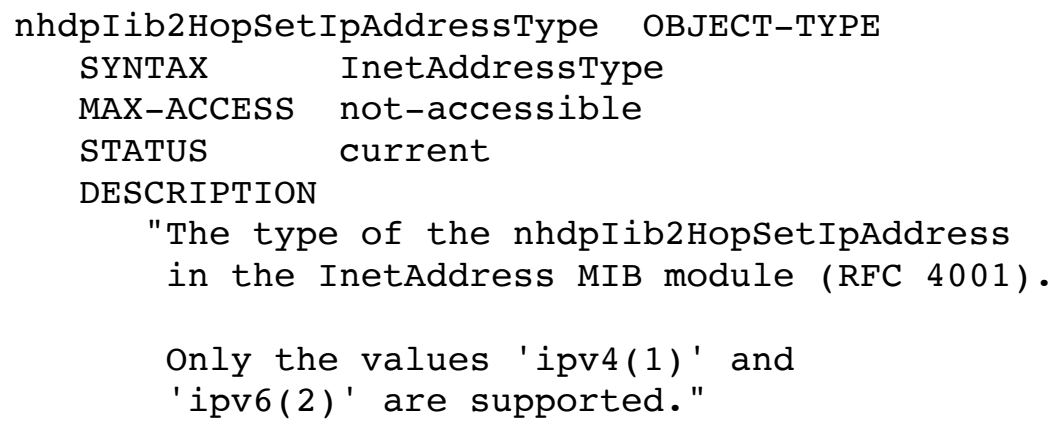

Herberg, et al.

Standards Track

[Page 44]

RFC 7939

The NHDP-MIB

August 2016

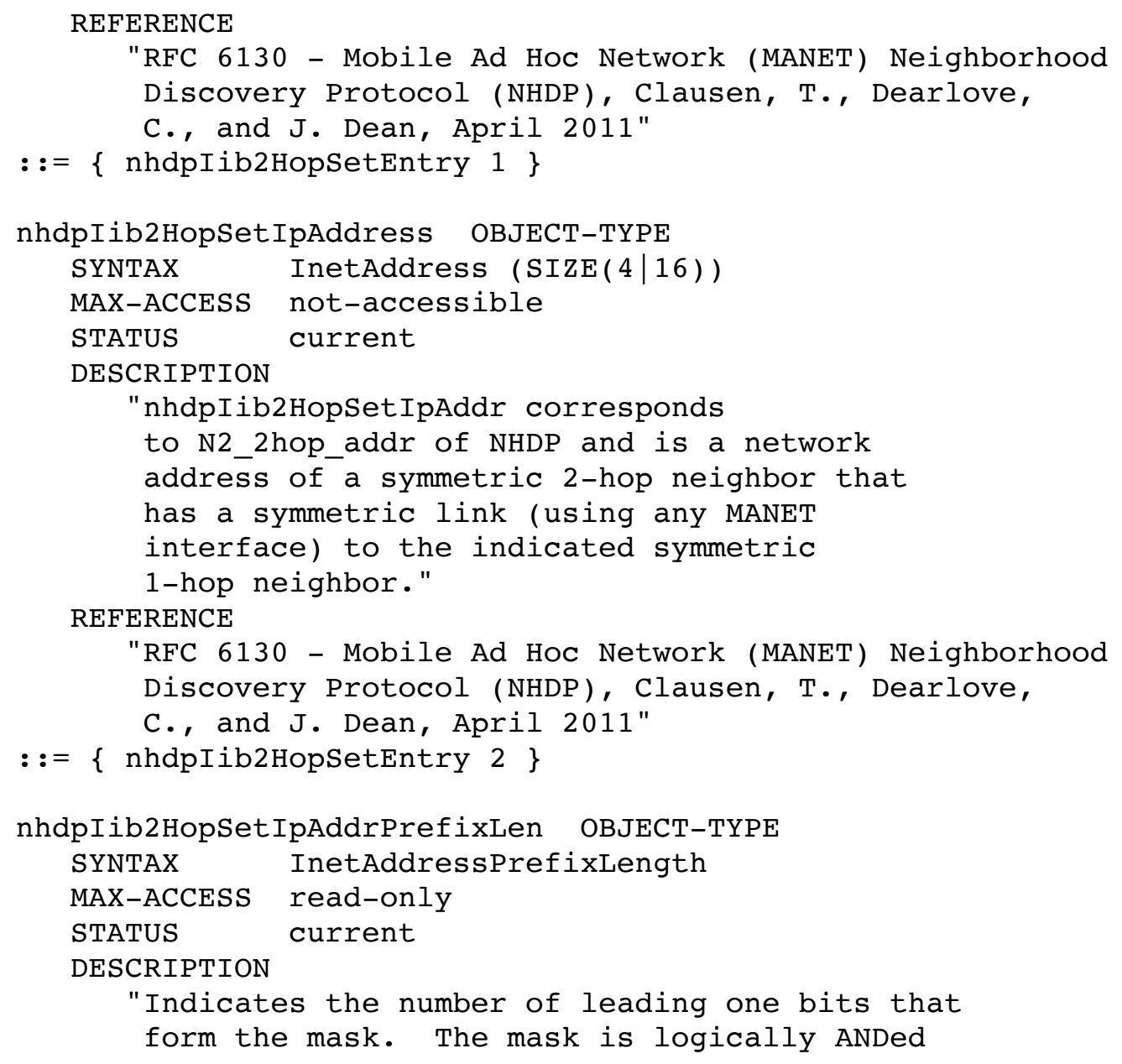


Network (MANET) Neighborhood Discovery Protocol (NHDP),

Dearlove, C., and T. Clausen, March 2015"

$::=\{$ nhdpIib2HopsetEntry 6$\}$

-- Neighbor Information Base (NIB)

-- Each router maintains a Neighbor Information Base

-- that records information about addresses of

-- current and recently symmetric 1-hop neighbors.

Herberg, et al.

RFC 7939
Standards Track

The NHDP-MIB
[Page 46]

August 2016

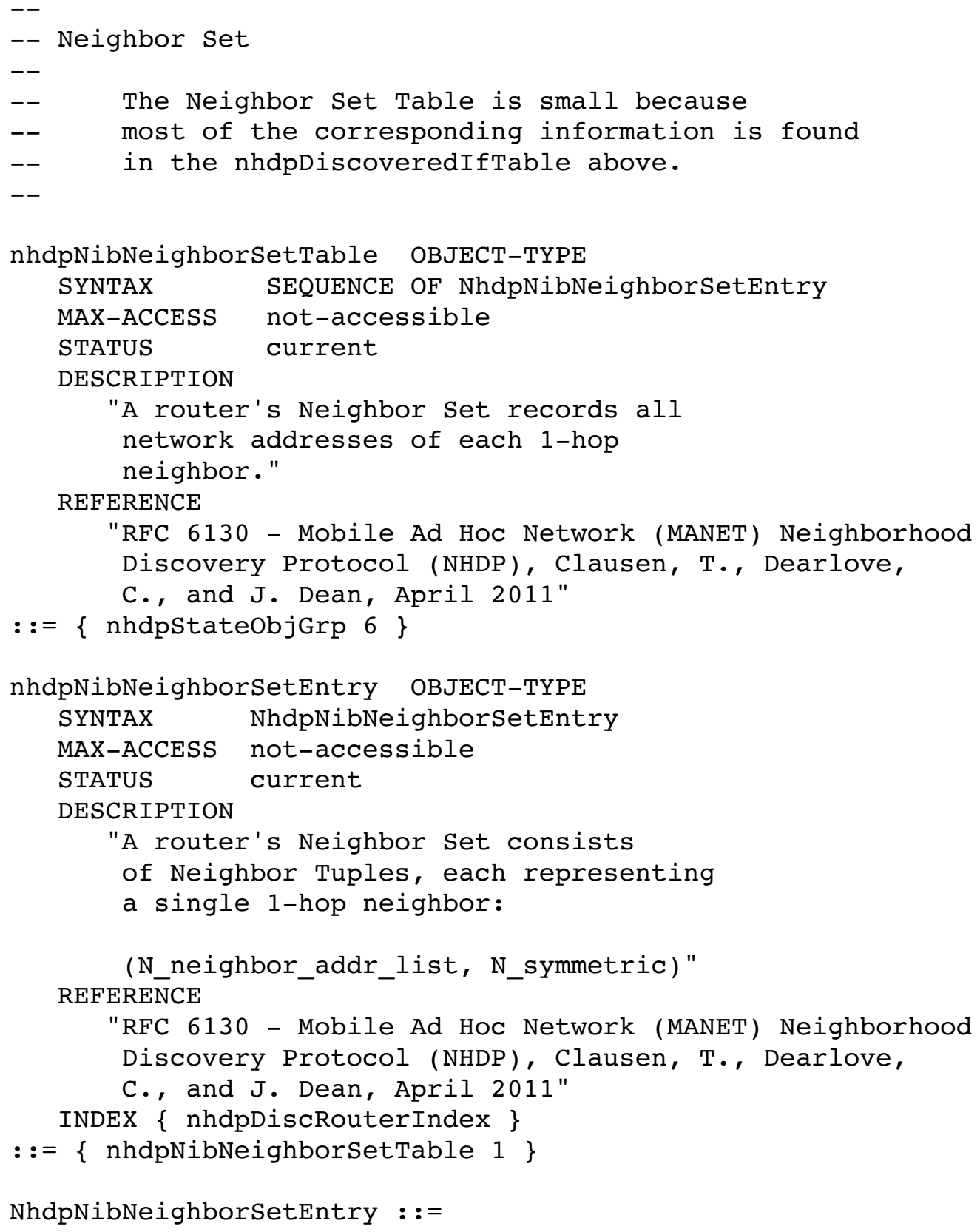




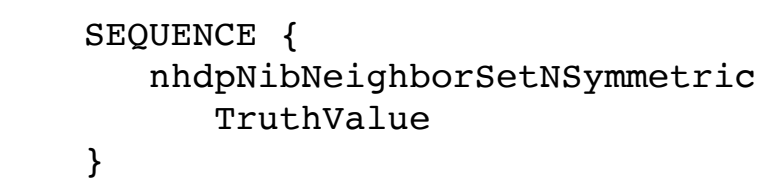

nhdpNibNeighborSetNSymmetric OBJECT-TYPE SYNTAX TruthValue MAX-ACCESS read-only

Herberg, et al.

RFC 7939
Standards Track

The NHDP-MIB
[Page 47]

August 2016

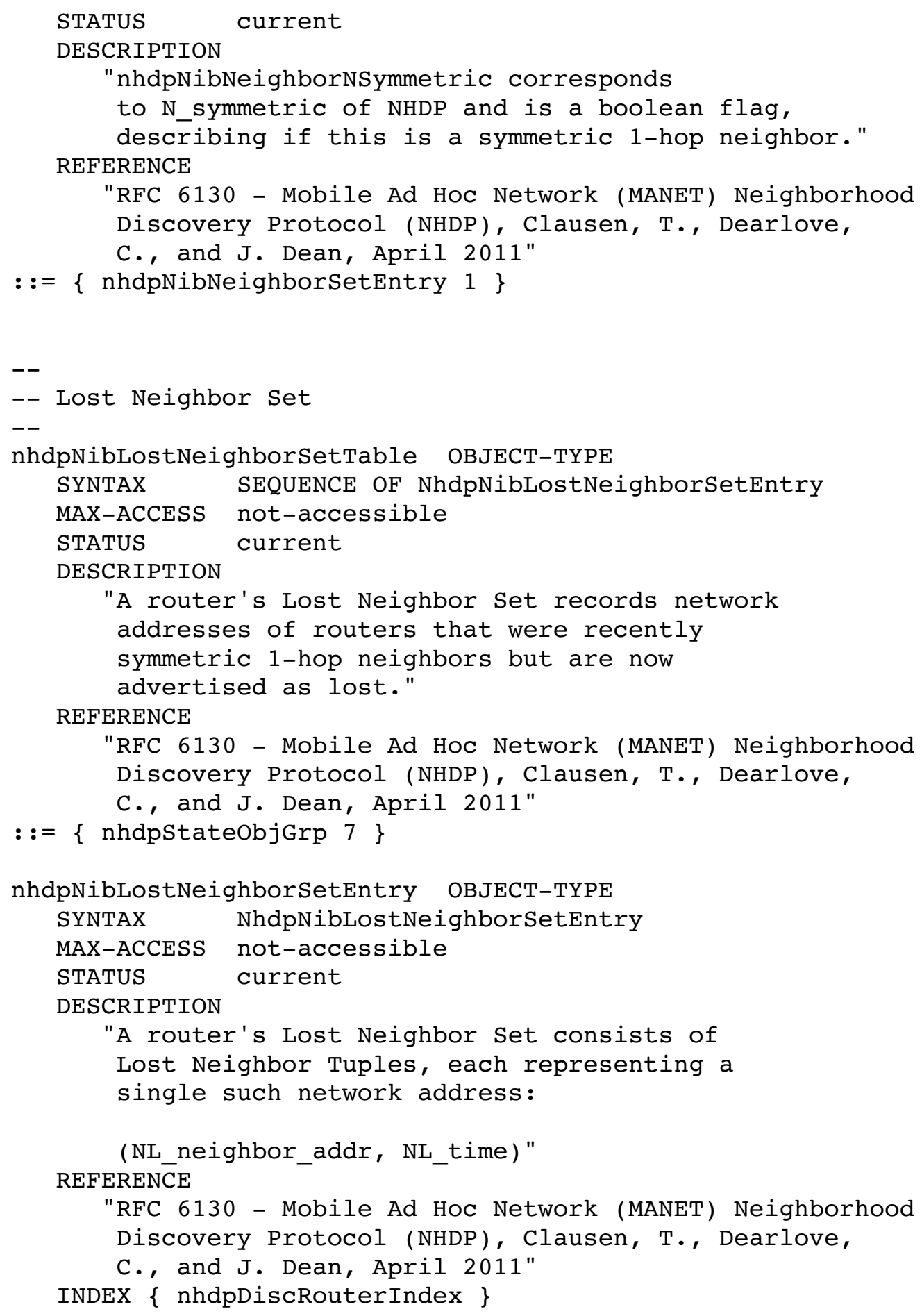


$::=\{$ nhdpNibLostNeighborSetTable 1$\}$

Herberg, et al.

RFC 7939
Standards Track

The NHDP-MIB
[Page 48]

August 2016

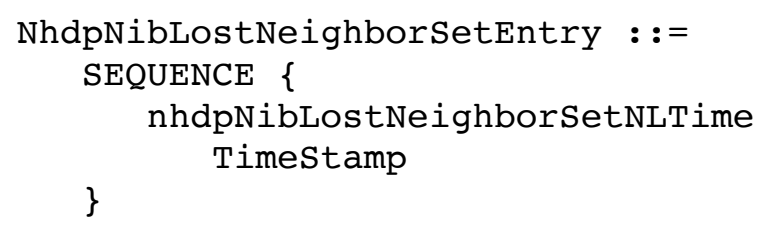

nhdpNibLostNeighborSetNLTime OBJECT-TYPE

$\begin{array}{ll}\text { SYNTAX } & \text { Timestamp } \\ \text { MAX-ACCESS } & \text { read-only } \\ \text { STATUS } & \text { current }\end{array}$

DESCRIPTION

"nhdpNibLostNeighborSetNLTime

specifies the value of sysupTime when this entry should expire and be removed from the nhdpNibLostNeighborSetTable."

REFERENCE

"RFC 6130 - Mobile Ad Hoc Network (MANET) Neighborhood Discovery Protocol (NHDP), Clausen, T., Dearlove, C., and J. Dean, April 2011"

$::=\{$ nhdpNibLostNeighborSetEntry 1$\}$

-- nhdpPerformanceObjGrp

-- Contains objects that help to characterize the performance of

-- the NHDP process, typically counters.

--

nhdpPerformanceobjGrp OBJECT IDENTIFIER ::= \{ nhdpobjects 3$\}$

-- Objects per local interface

--

nhdpInterfacePerfTable OBJECT-TYPE

SYNTAX SEQUENCE OF NhdpInterfacePerfEntry

MAX-ACCESS not-accessible

STATUS current

DESCRIPTION

"This table summarizes performance objects that are measured per local NHDP interface. nhdpIfPerfCounterDiscontinuityTime indicates the most recent occasion at which any one or more of this interface's counters listed in this table suffered a discontinuity." 
Herberg, et al.

RFC 7939
Standards Track

[Page 49]

The NHDP-MIB

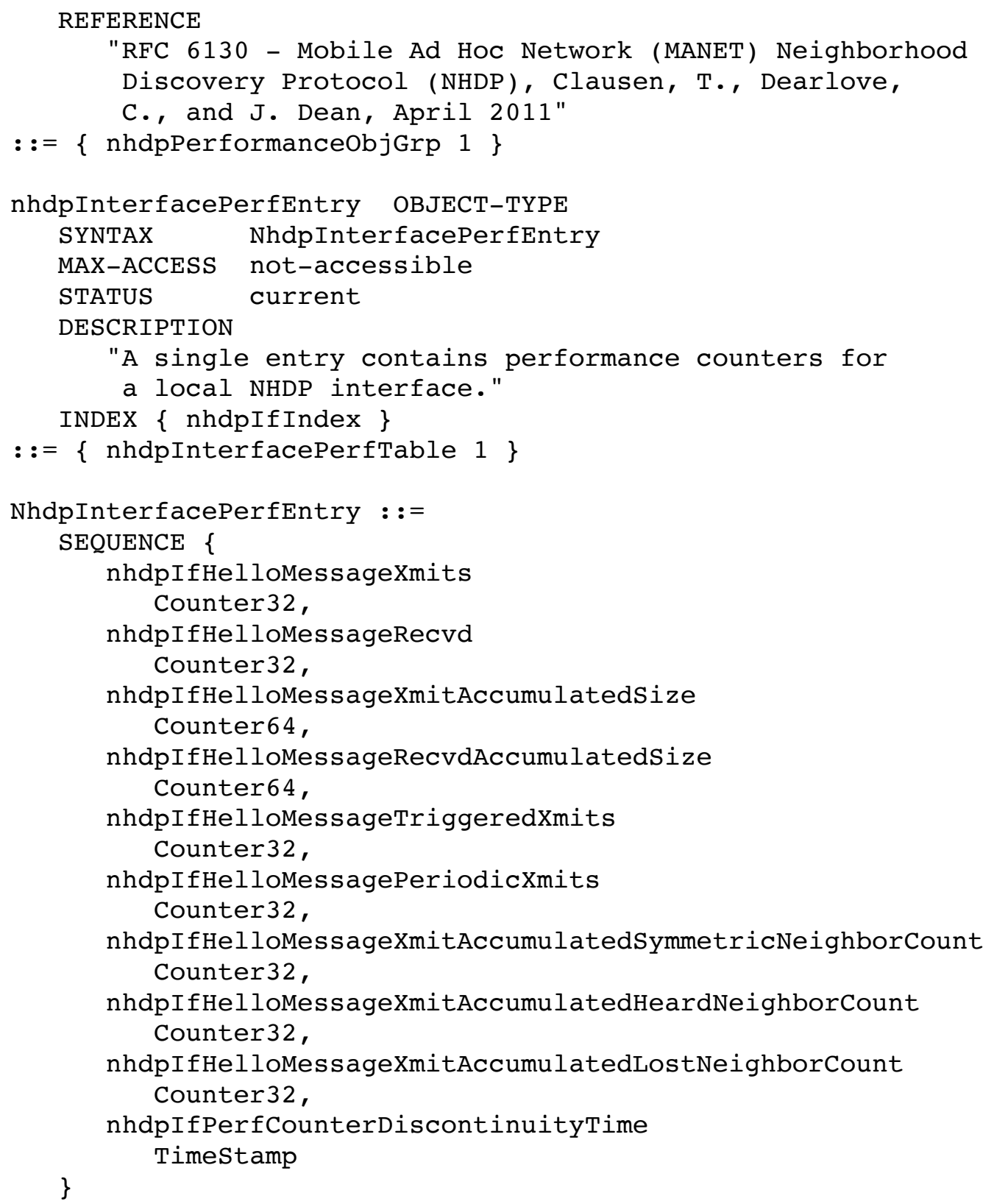




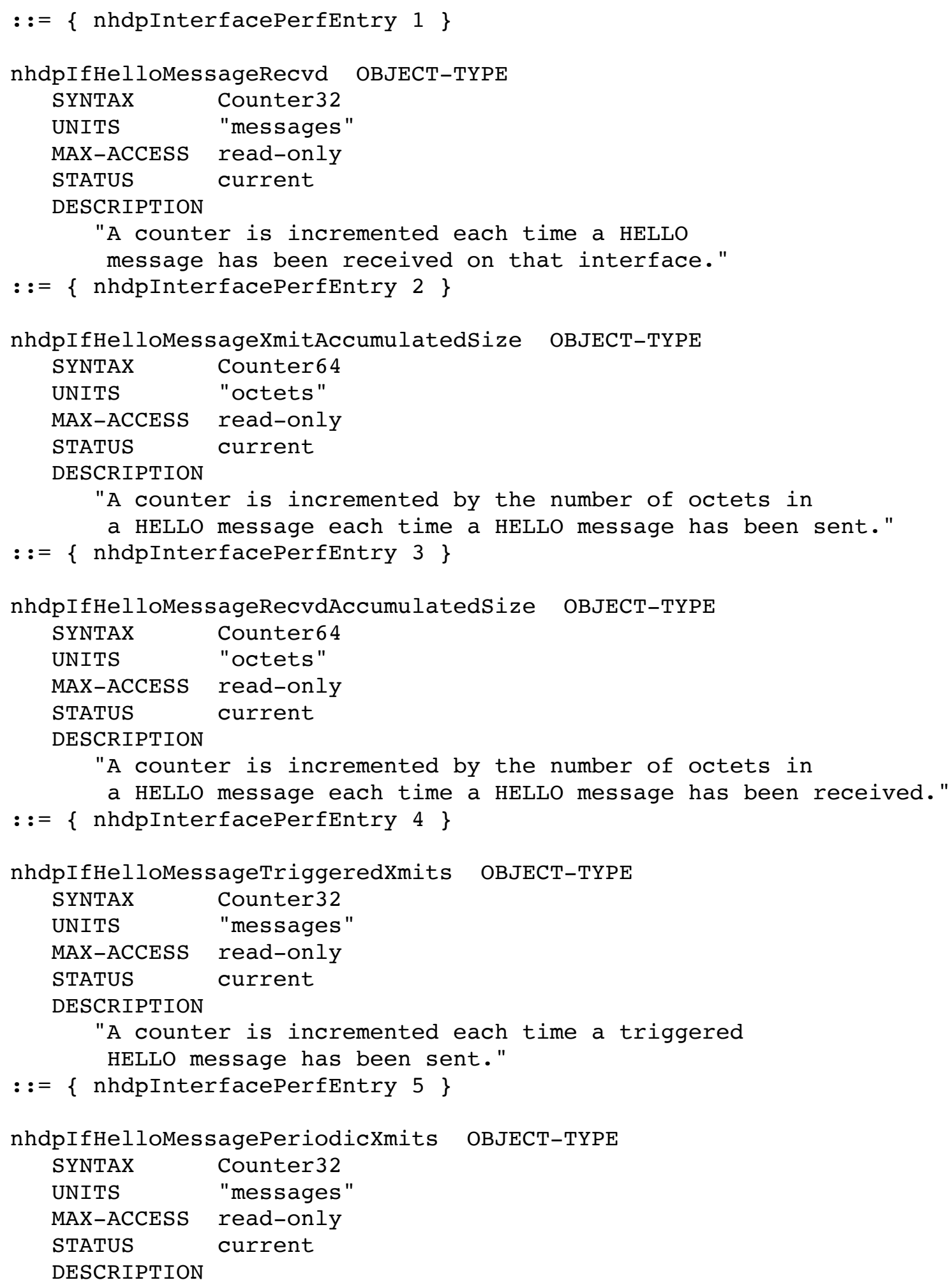

Herberg, et al.

RFC 7939
Standards Track

[Page 51]

The NHDP-MIB

"A counter is incremented each time a periodic HELLO message has been sent." $::=\{$ nhdpInterfacePerfEntry 6$\}$ 
nhdpIfHelloMessageXmitAccumulatedSymmetricNeighborCount OBJECT-TYPE

$\begin{array}{ll}\text { SYNTAX } & \text { Counter32 } \\ \text { UNITS } & \text { "neighbors" } \\ \text { MAX-ACCESS } & \text { read-only } \\ \text { STATUS } & \text { current } \\ \text { DESCRIPTION } & \end{array}$

"A counter is incremented by the number of advertised symmetric neighbors in a HELLO each time a HELLO message has been sent."

$::=\{$ nhdpInterfacePerfEntry 7$\}$

nhdpIfHelloMessageXmitAccumulatedHeardNeighborCount OBJECT-TYPE

$\begin{array}{ll}\text { SYNTAX } & \text { Counter32 } \\ \text { UNITS } & \text { "neighbors" } \\ \text { MAX-ACCESS } & \text { read-only } \\ \text { STATUS } & \text { current }\end{array}$

DESCRIPTION

"A counter is incremented by the number of advertised heard neighbors in a HELLO each time a HELLO message has been sent."

$::=\{$ nhdpInterfacePerfentry 8$\}$

nhdpIfHelloMessageXmitAccumulatedLostNeighborCount OBJECT-TYPE

$\begin{array}{ll}\text { SYNTAX } & \text { Counter32 } \\ \text { UNITS } & \text { "neighbors" } \\ \text { MAX-ACCESS } & \text { read-only } \\ \text { STATUS } & \text { current }\end{array}$

DESCRIPTION

"A counter is incremented by the number of advertised lost neighbors in a HELLO each time a HELLO message has been sent."

$::=\{$ nhdpInterfacePerfEntry 9$\}$

nhdpIfPerfCounterDiscontinuityTime OBJECT-TYPE

$\begin{array}{ll}\text { SYNTAX } & \text { Timestamp } \\ \text { MAX-ACCESS } & \text { read-only } \\ \text { STATUS } & \text { current }\end{array}$

DESCRIPTION

"The value of sysupTime on the most recent occasion at which any one or more of this interface's counters suffered a discontinuity. If no such discontinuities have occurred since the last reinitialization of the local management subsystem, then this object contains a zero value."

$::=\{$ nhdpInterfacePerfEntry 10$\}$

Herberg, et al.

RFC 7939
Standards Track

The NHDP-MIB
[Page 52]

August 2016 
router receives a packet from that interface

of the neighbor."

REFERENCE

"RFC 6130 - Mobile Ad Hoc Network (MANET) Neighborhood

Discovery Protocol (NHDP), Clausen, T., Dearlove,

C., and J. Dean, April 2011"

$::=\{$ nhdpDiscIfSetPerfEntry 2$\}$

-- Objects concerning the Neighbor set

- -

nhdpNibNeighborSetchanges OBJECT-TYPE

SYNTAX Counter32

UNITS "changes"

MAX-ACCESS read-only

STATUS current

DESCRIPTION

"This counter increments each time the Neighbor set changes.

A change occurs whenever a new Neighbor Tuple has been

added, a Neighbor Tuple has been removed, or any entry of

a Neighbor Tuple has been modified."

$::=\{$ nhdpPerformanceobjGrp 3$\}$

-- Objects per discovered neighbor

--

nhdpDiscNeighborSetPerfTable OBJECT-TYPE

SYNTAX SEQUENCE OF NhdpDiscNeighborSetPerfEntry

MAX-ACCESS not-accessible

STATUS current

Herberg, et al.

Standards Track

[Page 54]

RFC 7939

The NHDP-MIB

August 2016

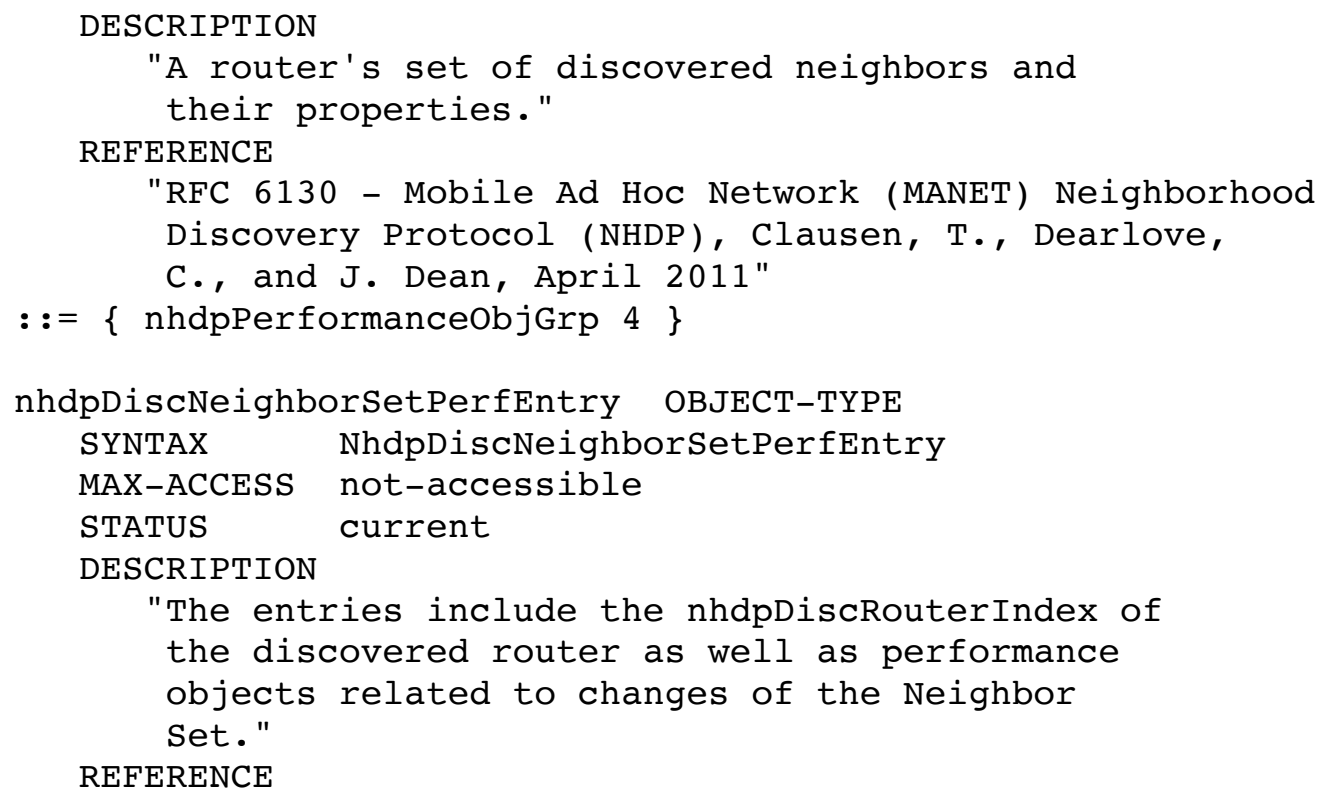



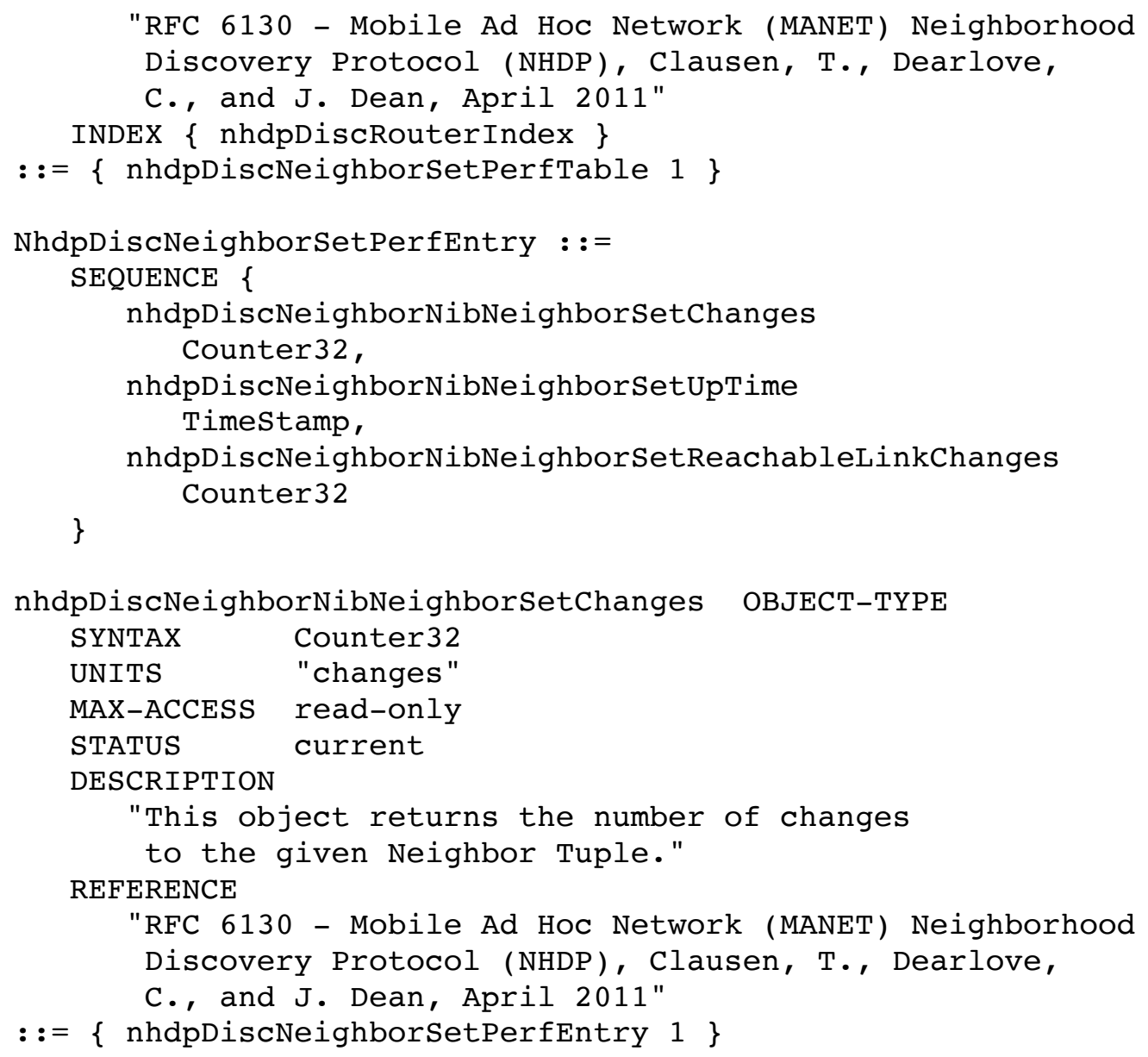

Herberg, et al.

RFC 7939
Standards Track

The NHDP-MIB
[Page 55]

August 2016

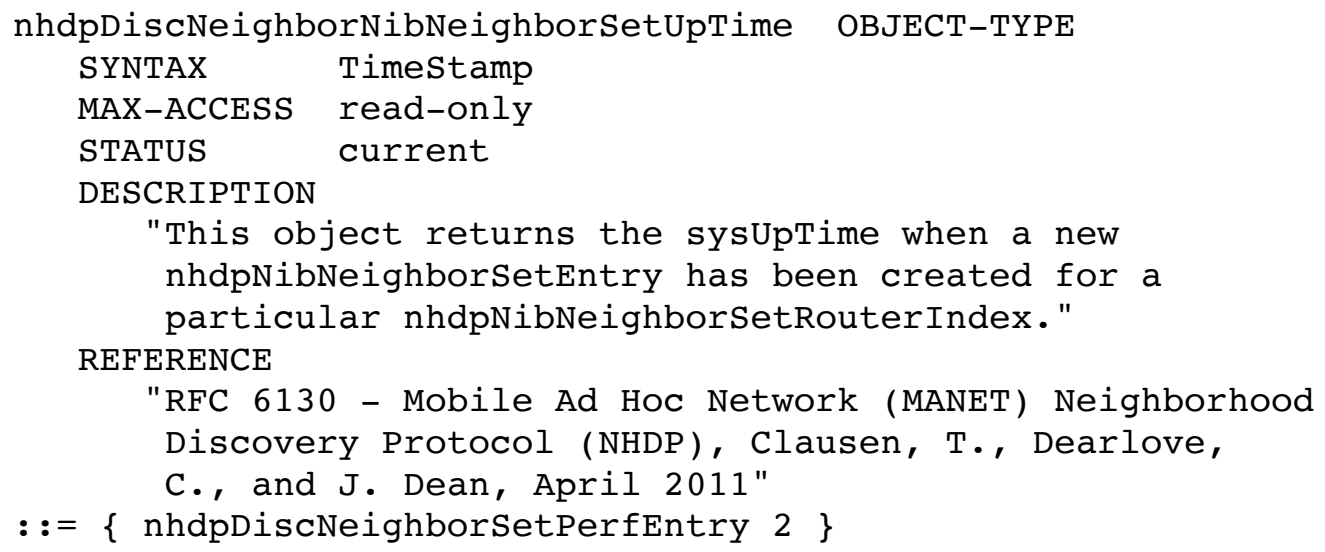


i.e., a corresponding Link Tuple is added or removed

from the set of all corresponding Link Tuples."

REFERENCE

"RFC 6130 - Mobile Ad Hoc Network (MANET) Neighborhood

Discovery Protocol (NHDP), Clausen, T., Dearlove,

C., and J. Dean, April 2011"

$::=\{$ nhdpDiscNeighborSetPerfEntry 3$\}$

-- Objects per discovered 2-hop neighbor

--

nhdpIib2HopsetPerfTable OBJECT-TYPE

SYNTAX SEQUENCE OF NhdpIib2HopsetPerfEntry

MAX-ACCESS not-accessible

STATUS current

DESCRIPTION

"This table contains performance objects per

discovered 2-hop neighbor."

REFERENCE

"RFC 6130 - Mobile Ad Hoc Network (MANET) Neighborhood

Discovery Protocol (NHDP), Clausen, T., Dearlove,

C., and J. Dean, April 2011"

$::=\{$ nhdpPerformanceobjGrp 5$\}$

Herberg, et al.

RFC 7939
Standards Track

The NHDP-MIB
[Page 56]

August 2016

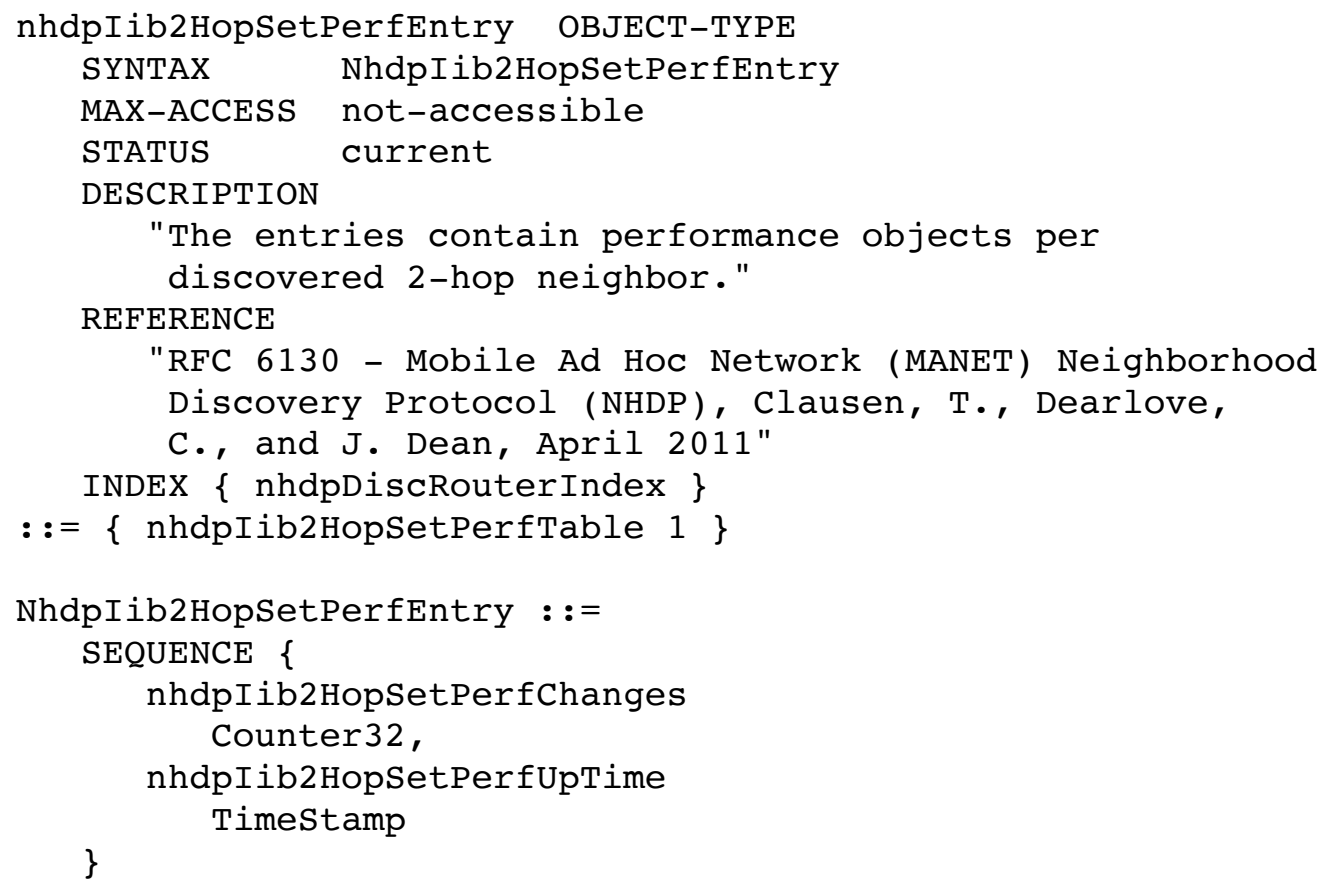




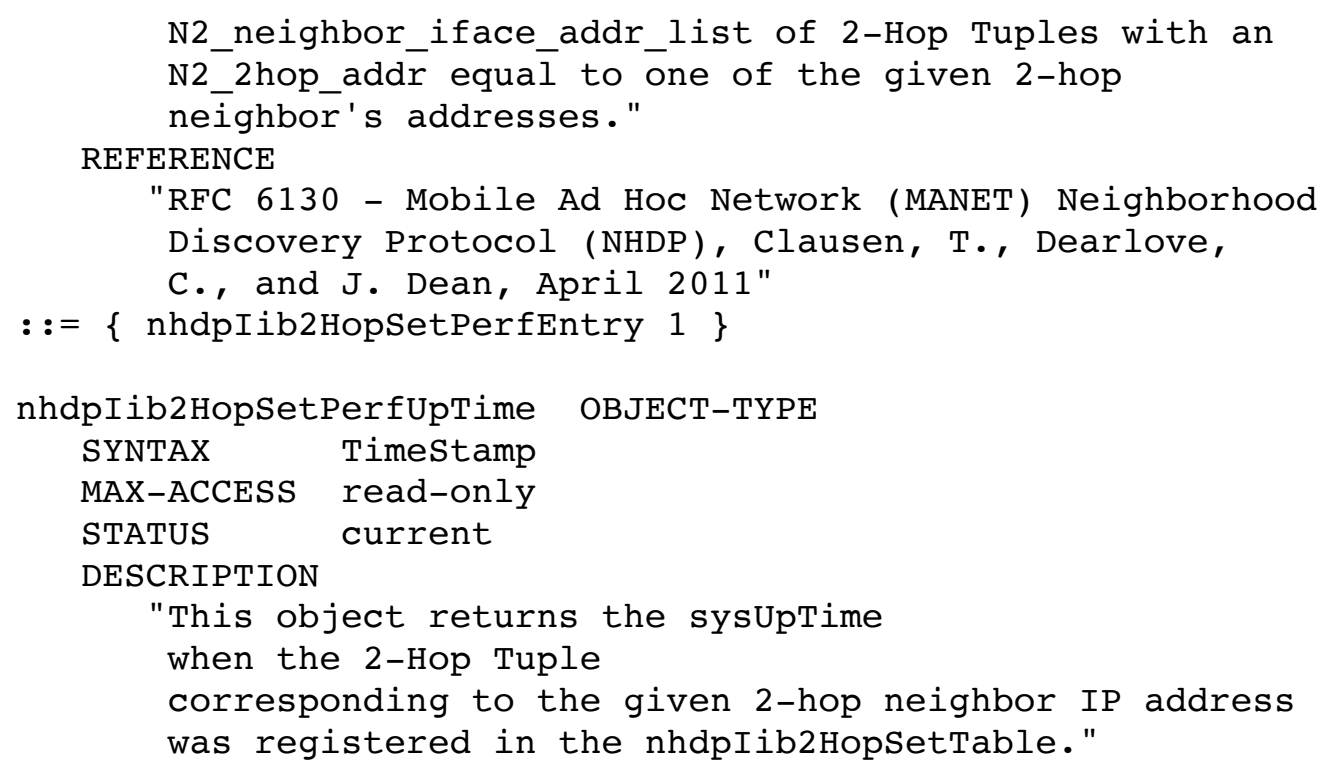

Herberg, et al.

RFC 7939
Standards Track

The NHDP-MIB
[Page 57]

August 2016

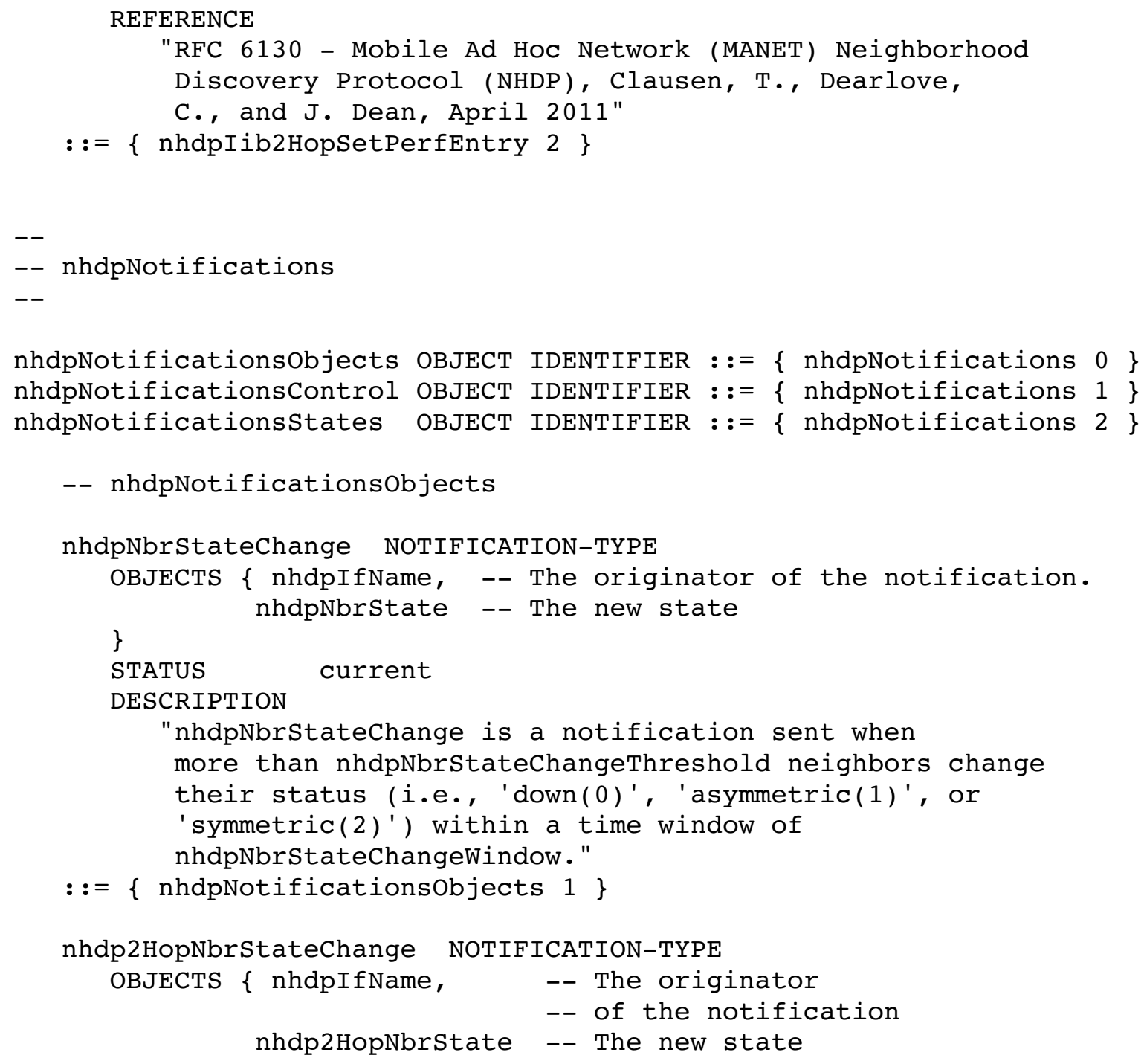

-- nhdpNotifications

$--$

nhdpNotificationsObjects OBJECT IDENTIFIER : := \{ nhdpNotifications 0$\}$ nhdpNotificationsControl OBJECT IDENTIFIER ::= \{ nhdpNotifications 1$\}$ nhdpNotificationsstates OBJECT IDENTIFIER ::= \{ nhdpNotifications 2$\}$

-- nhdpNotificationsobjects 


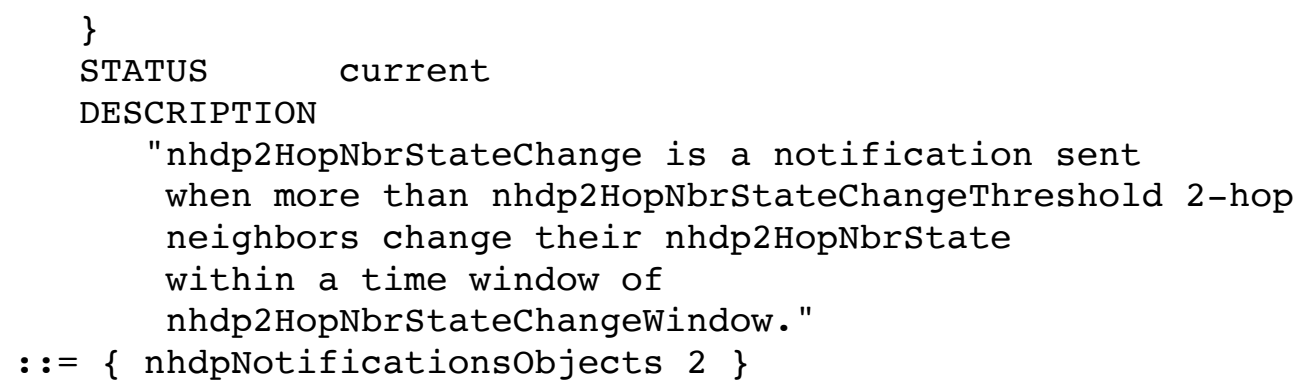

nhdpIfStateChange NOTIFICATION-TYPE

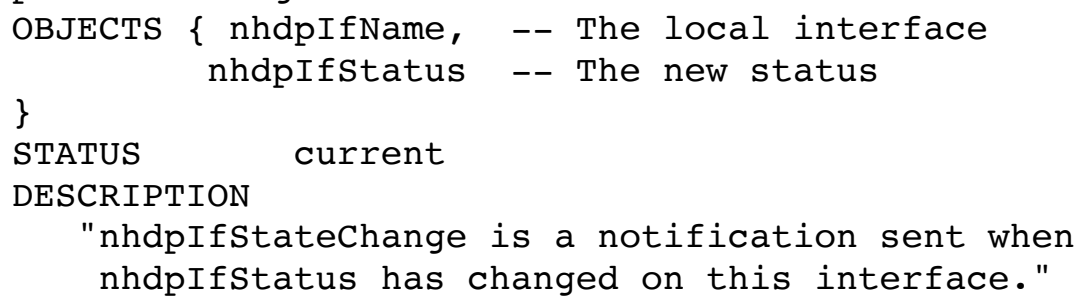

-- nhdpNotificationsControl

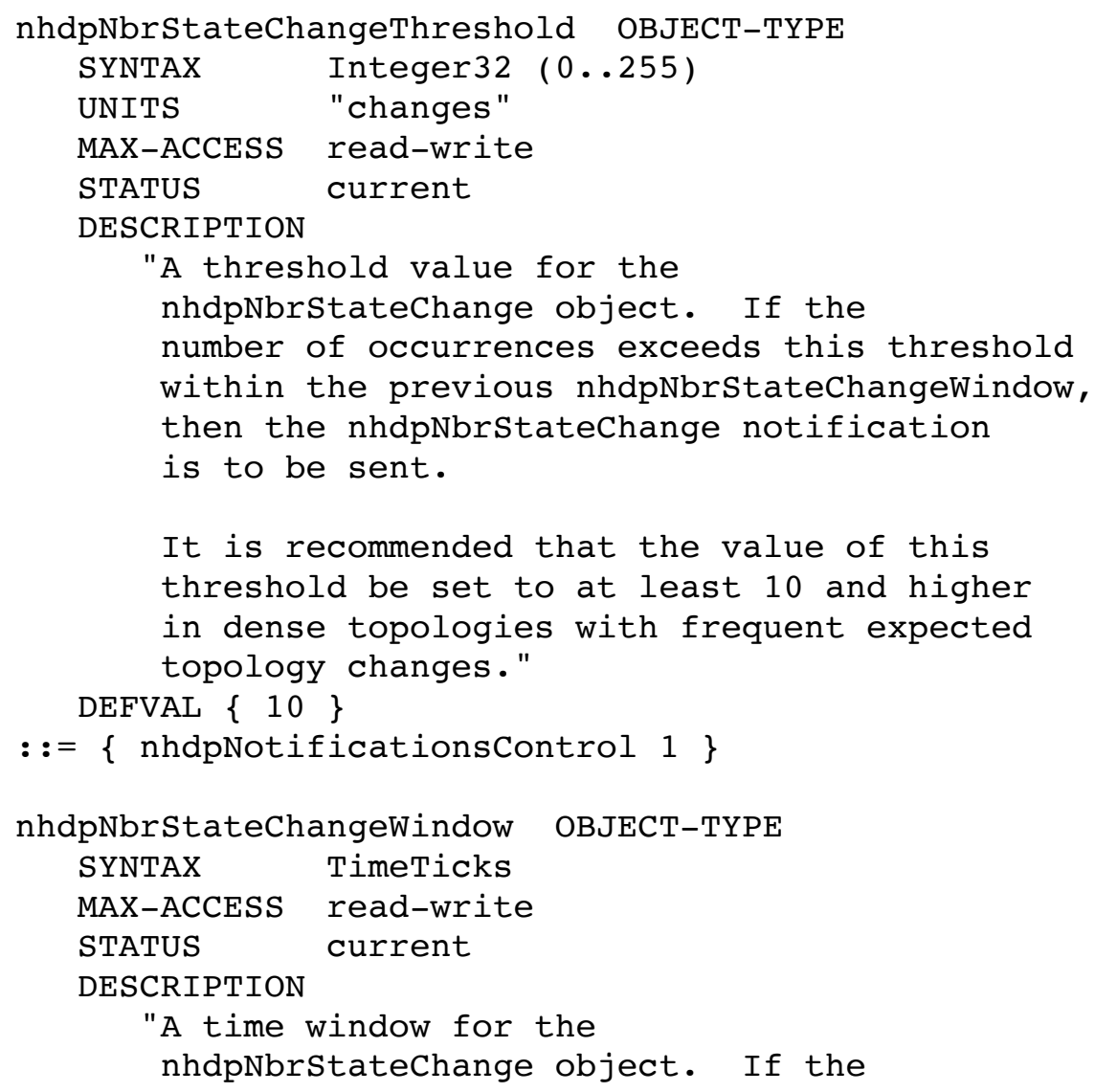


number of occurrences exceeds the

nhdpNbrStatechangeThreshold

within the previous nhdpNbrstatechangewindow,

then the nhdpNbrStatechange notification

is to be sent.

It is recommended that the value for this

window be set to at least 5 times the

nhdpHelloInterval.

Herberg, et al.

RFC 7939
Standards Track

The NHDP-MIB
[Page 59]

August 2016

This object represents the time in hundredths

of a second."

DEFVAL $\{1000\}$

$::=\{$ nhdpNotificationsControl 2$\}$

nhdp2HopNbrStateChangeThreshold OBJECT-TYPE

SYNTAX Integer32 (0..255)

UNITS "changes"

MAX-ACCESS read-write

STATUS current

DESCRIPTION

"A threshold value for the

nhdp2HopNbrStatechange object. If the

number of occurrences exceeds this threshold

within the previous nhdp2HopNbrStatechangewindow,

then the nhdp2HopNbrStatechange notification

is to be sent.

It is recommended that the value of this

threshold be set to at least 10 and higher

when topologies are expected to be highly dynamic."

DEFVAL $\{10\}$

$::=\{$ nhdpNotificationsControl 3$\}$

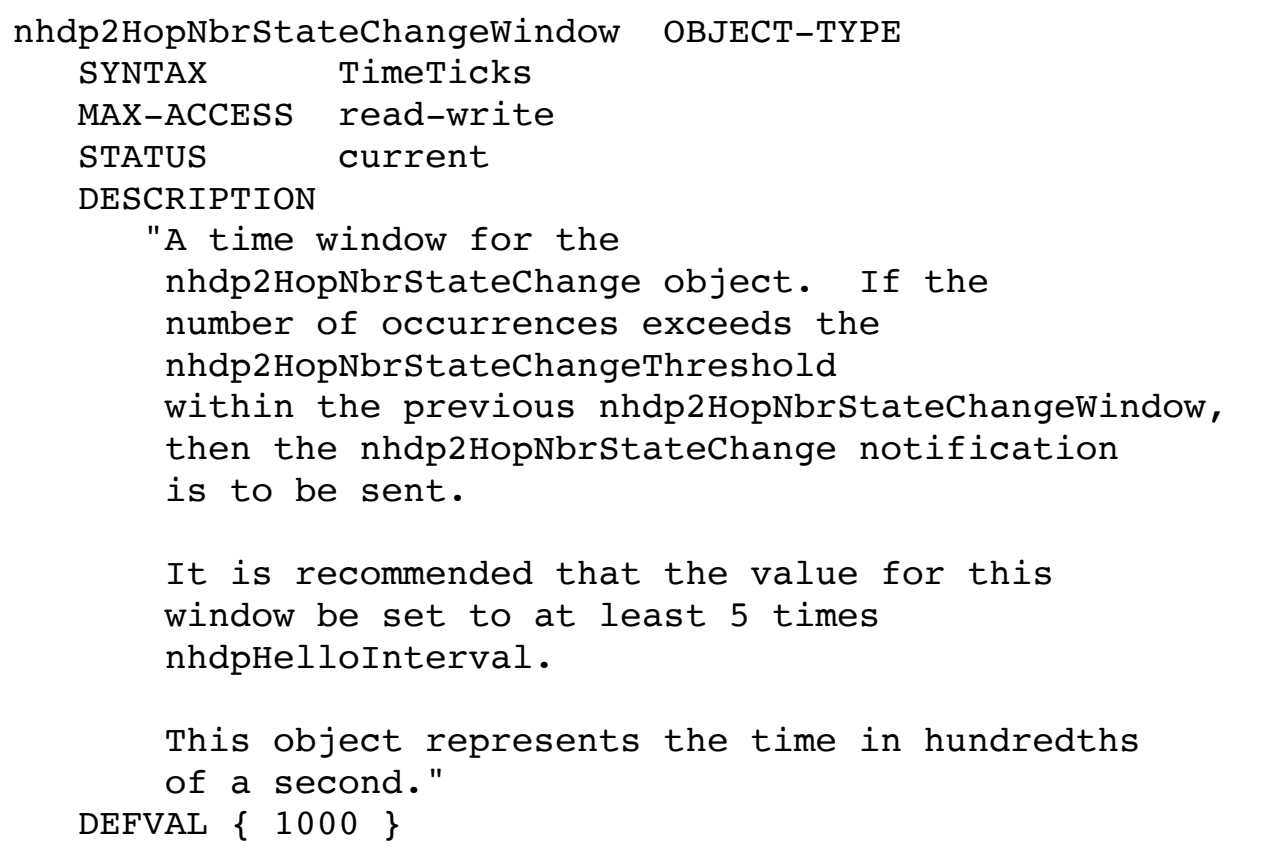


$::=\{$ nhdpNotificationsControl 4$\}$

Herberg, et al.

RFC 7939
Standards Track

The NHDP-MIB
[Page 60]

August 2016

-- nhdpNotificationstates

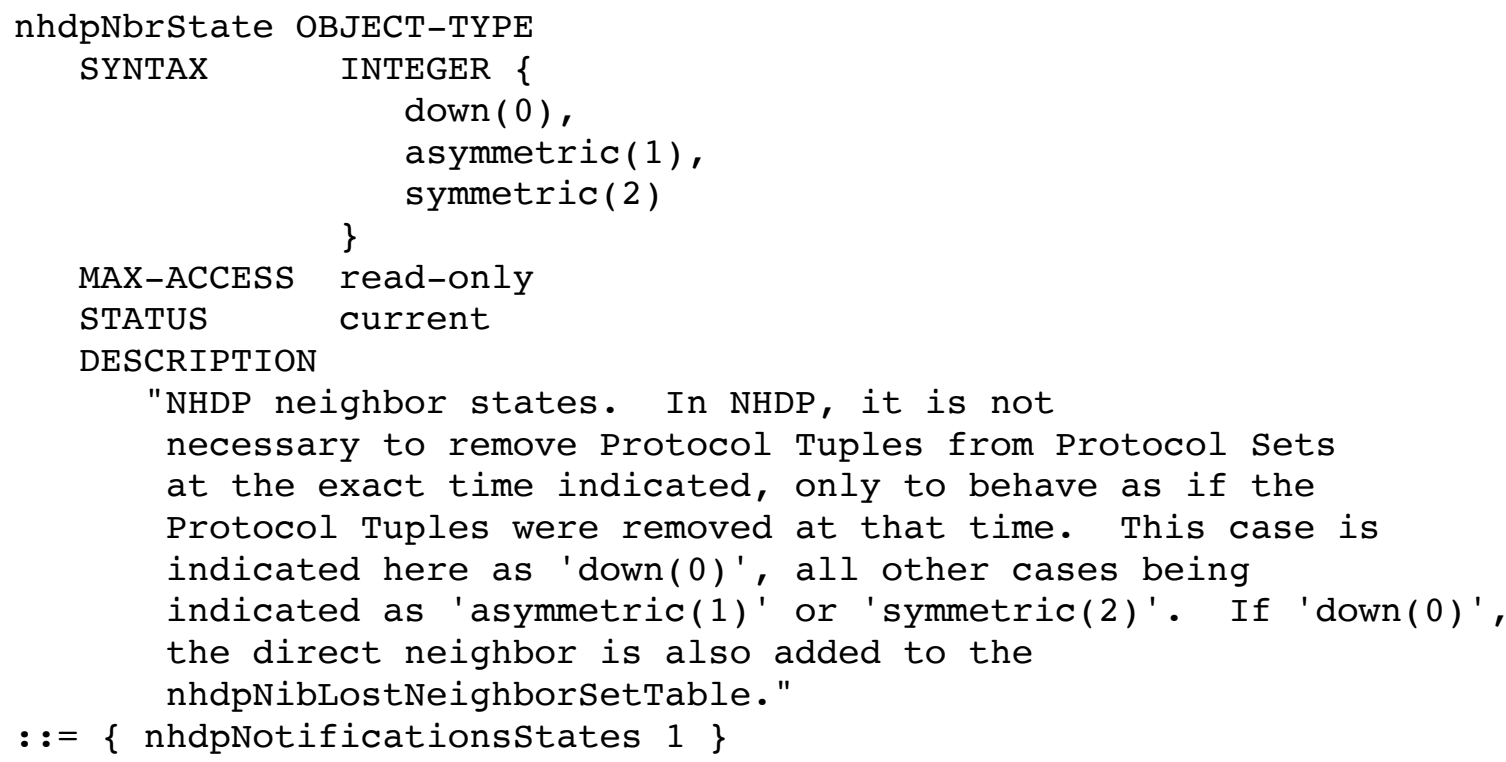

nhdp2 HopNbrState OBJECT-TYPE

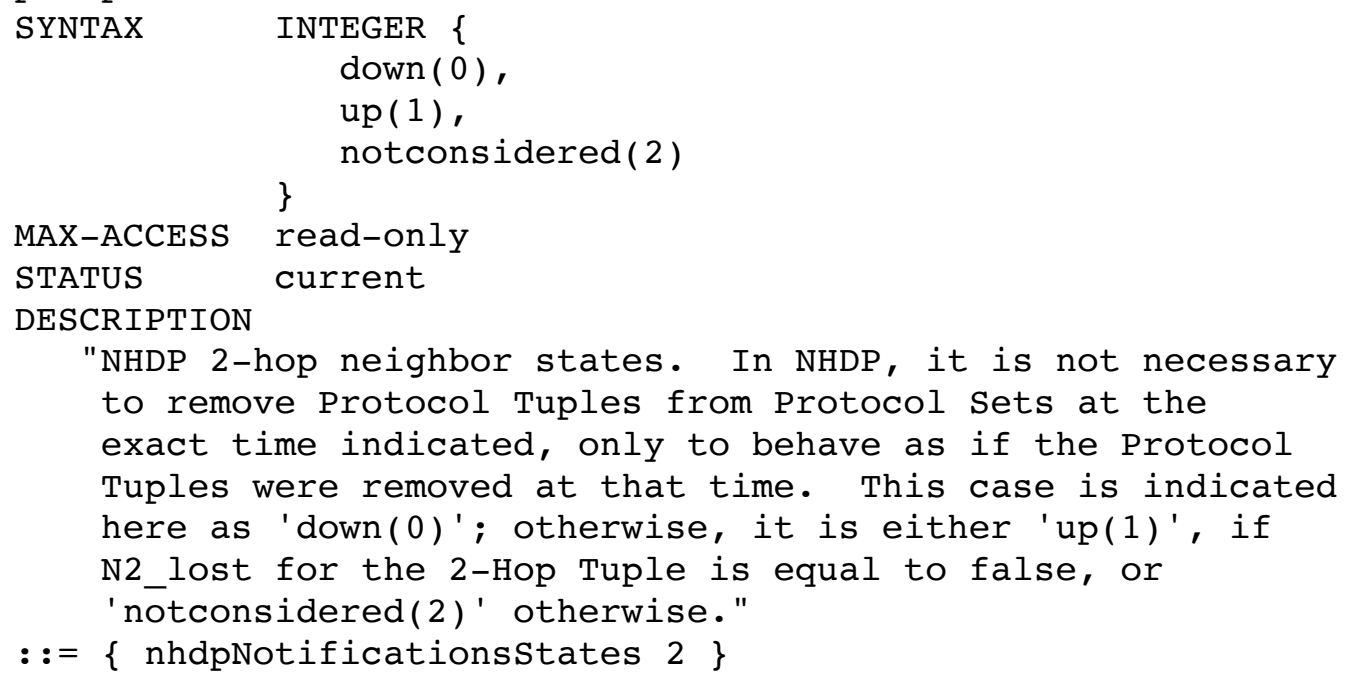

-- nhdpconformance information

nhdpCompliances nhdpMIBGroups
OBJECT IDENTIFIER : := $\{$ nhdpConformance 1$\}$ OBJECT IDENTIFIER : := \{ nhdpConformance 2$\}$ 
Herberg, et al.

Standards Track

The NHDP-MIB
[Page 61]

August 2016

-- Compliance Statements

nhdpBasicCompliance MODULE-COMPLIANCE

STATUS current

DESCRIPTION

"The basic implementation requirements for managed network entities that implement NHDP."

MODULE -- this module

MANDATORY-GROUPS \{ nhdpConfigurationGroup \}

$::=\{$ nhdpCompliances 1$\}$

nhdpFullCompliance2 MODULE-COMPLIANCE

STATUS current

DESCRIPTION

"The full implementation requirements for managed network entities that implement NHDP."

MODULE -- this module

MANDATORY-GROUPS \{ nhdpConfigurationGroup, nhdpstateGroup2 , nhdpNotificationobjectGroup, nhdpNotificationGroup,

\} nhdpPerformanceGroup

$::=\{$ nhdpCompliances 3$\}$

-- Units of Conformance

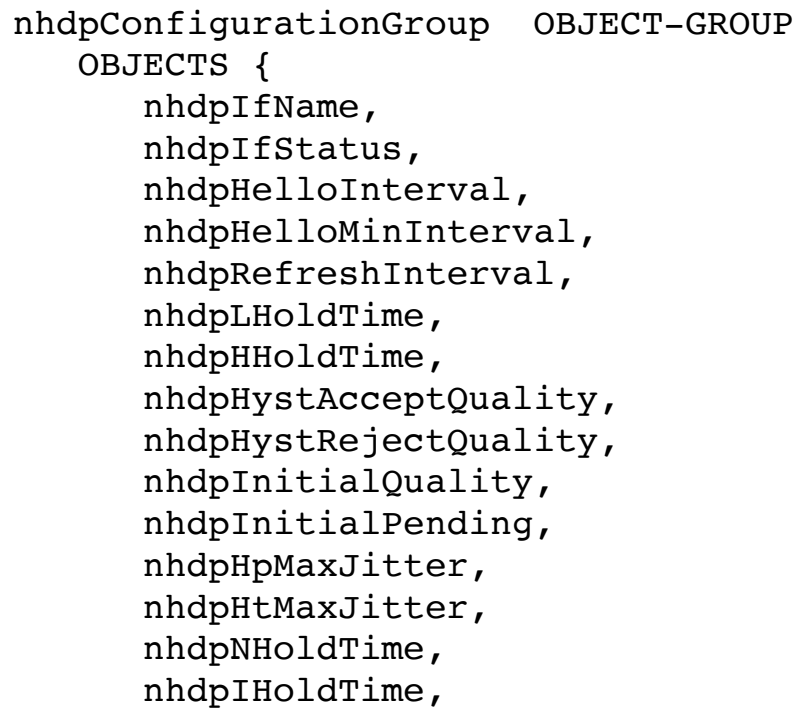




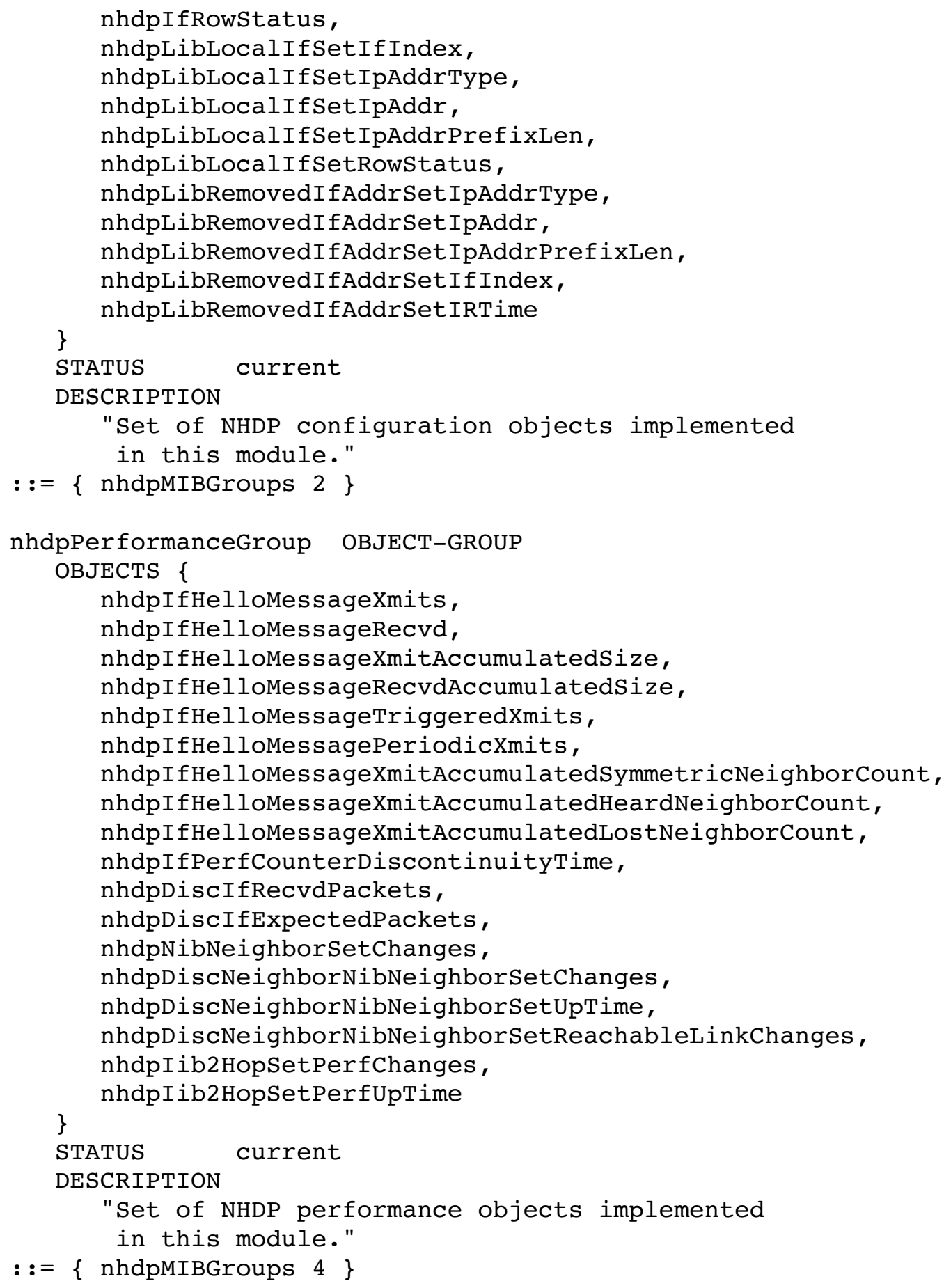

Herberg, et al.

Standards Track

[Page 63]

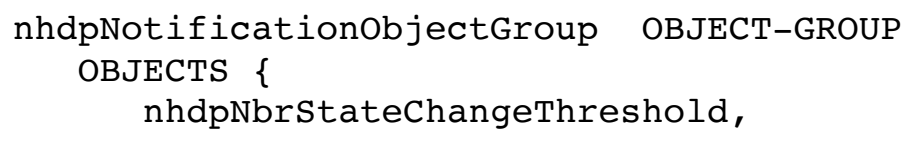


nhdpNbrstatechangewindow,

nhdp2 HopNbrStateChangeThreshold, nhdp2 HopNbrStatechangewindow, nhdpNbrstate, nhdp2HopNbrState

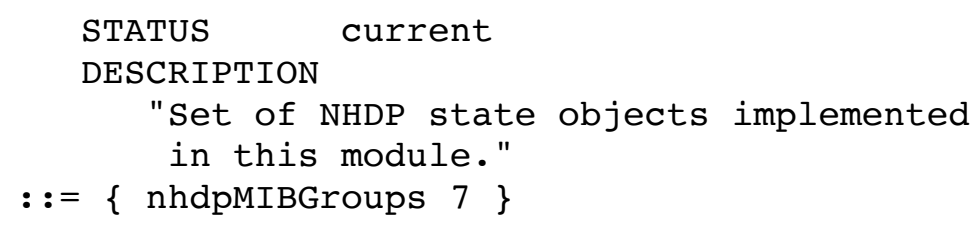


-- Deprecated compliance statements and groups

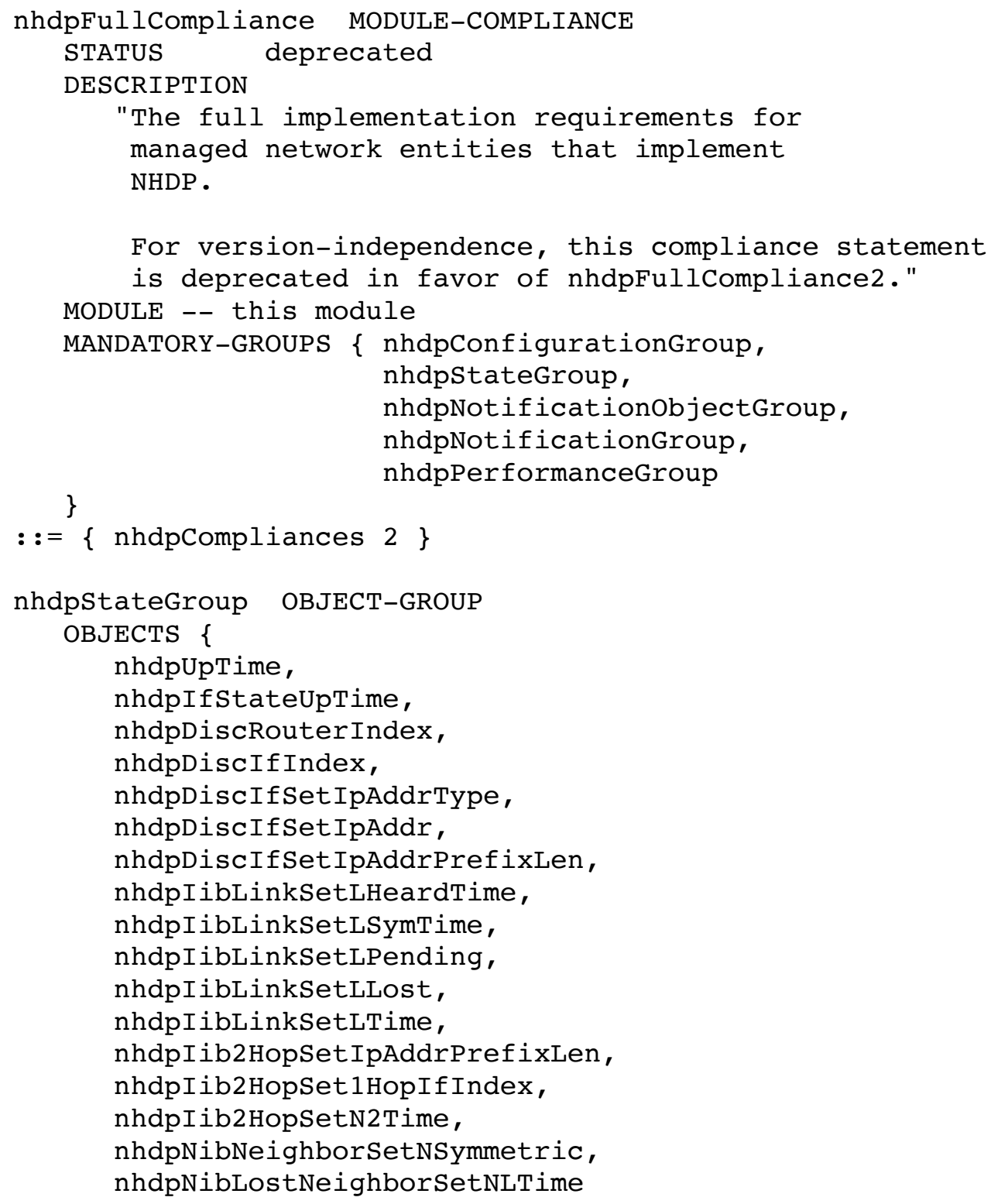

$$
\}
$$

END 


\section{Security Considerations}

This MIB module defines objects for the configuration, monitoring, and notification of the Mobile Ad Hoc Network (MANET) Neighborhood Discovery Protocol (NHDP) [RFC6130]. NHDP allows routers to acquire topological information up to two hops away by virtue of exchanging HELLO messages. The information acquired by NHDP may be used by routing protocols. The neighborhood information, exchanged between routers using NHDP, serves these routing protocols as a baseline for calculating paths to all destinations in the MANET, relay set selection for network-wide transmissions, etc.

There are a number of management objects defined in this MIB module with a MAX-ACCESS clause of read-write and/or read-create. Such objects may be considered sensitive or vulnerable in some network environments. The support for SET operations in a non-secure environment without proper protection opens devices to attack. These are the tables and objects and their sensitivity/vulnerability:

o nhdpIfStatus - This writable object turns on or off the NHDP process for the specified interface. If disabled, higher-level protocol functions, e.g., routing, would fail, causing networkwide disruptions.

- nhdpHelloInterval, nhdpHellominInterval, and nhdpRefreshInterval These writable objects control the rate at which HELLO messages are sent on an interface. If set at too high a rate, this could represent a form of denial-of-service (DoS) attack by overloading interface resources.

o nhdpHystAcceptQuality, nhdpHystRejectQuality, nhdpInitialQuality, and nhdpInitialpending - These writable objects affect the perceived quality of the NHDP links and hence the overall stability of the network. If improperly set, these settings could result in network-wide disruptions.

Herberg, et al.

RFC 7939
Standards Track

The NHDP-MIB
[Page 66]

August 2016

- nhdpInterfaceTable - This table contains writable objects that affect the overall performance and stability of the NHDP process. Failure of the NHDP process would result in network-wide failure. Particularly sensitive objects from this table are discussed in the previous list items. This is the only table in the NHDP-MIB module with writable objects.

Some of the readable objects in this MIB module (i.e., objects with a MAX-ACCESS other than not-accessible) may be considered sensitive or vulnerable in some network environments. It is thus important to control even GET and/or NOTIFY access to these objects and possibly to even encrypt the values of these objects when sending them over the network via SNMP. These are the tables and objects and their sensitivity/vulnerability:

o nhdpDiscIfSetTable - The object contains information on discovered neighbors, specifically their IP address in the

nhdpDiscIfSetIpAddr object. This information provides an 
adversary broad information on the members of the MANET, located within this single table. This information can be used to expedite attacks on the other members of the MANET without having to go through a laborious discovery process on their own. This object is the index into the table and has a MAX-ACCESS of 'not-accessible'. However, this information can be exposed using SNMP operations.

MANET technology is often deployed to support communications of emergency services or military tactical applications. In these applications, it is imperative to maintain the proper operation of the communications network and to protect sensitive information related to its operation. Therefore, it is RECOMMENDED to provide support for the Transport Security Model (TSM) [RFC5591] in combination with TLS/DTLS [RFC6353].

SNMP versions prior to SNMPv3 did not include adequate security. Even if the network itself is secure (for example by using IPsec), there is no control as to who on the secure network is allowed to access and GET/SET (read/change/create/delete) the objects in this MIB module.

Implementations SHOULD provide the security features described by the SNMPv3 framework (see [RFC3410]), and implementations claiming compliance to the SNMPV3 standard MUST include full support for authentication and privacy via the User-based Security Model (USM) [RFC3414] with the AES cipher algorithm [RFC3826]. Implementations MAY also provide support for the Transport Security Model (TSM) [RFC5591] in combination with a secure transport such as SSH [RFC5592] or TLS/DTLS [RFC6353].

Herberg, et al.

RFC 7939
Standards Track

The NHDP-MIB
[Page 67]

August 2016

Further, deployment of SNMP versions prior to SNMPv3 is NOT RECOMMENDED. Instead, it is RECOMMENDED to deploy SNMPv3 and to enable cryptographic security. It is then a customer/operator responsibility to ensure that the SNMP entity giving access to an instance of this MIB module is properly configured to give access to the objects only to those principals (users) that have legitimate rights to indeed GET or SET (change/create/delete) them.

9. Applicability statement

This document describes objects for configuring parameters of the Mobile Ad Hoc Network (MANET) Neighborhood Discovery Protocol (NHDP) [RFC6130] process on a router. This MIB module, denoted NHDP-MIB, also reports state, performance information, and notifications. This section provides some examples of how this MIB module can be used in MANET network deployments.

NHDP is designed to allow routers to automatically discover and track routers one hop remote (denoted "neighbors") and routers two hops remote (denoted "2-hop neighbors"). This information is used by other MANET protocols in operation on the router to perform routing, multicast forwarding, and other functions with ad hoc and mobile networks. In the following, three example scenarios are listed where 
this MIB module is useful:

- For a Parking Lot Initial Configuration Situation - It is common for the vehicles comprising the MANET being forward deployed at a remote location, e.g., the site of a natural disaster, to be offloaded in a parking lot where an initial configuration of the networking devices is performed. The configuration is loaded into the devices from a fixed location Network Operations Center (NOC) at the parking lot, and the vehicles are stationary at the parking lot while the configuration changes are made. Standards-based methods for configuration management from the co-located Noc are necessary for this deployment option.

- For Mobile Vehicles with Low-Bandwidth Satellite Link to a Fixed NOC - Here, the vehicles carrying the MANET routers carry multiple wireless interfaces, one of which is a relatively low-bandwidth, on-the-move satellite connection that interconnects a fix NOC to the nodes of the MANET. Standards-based methods for monitoring and fault management from the fixed NOC are necessary for this deployment option.

- For Fixed NOC and Mobile Local Manager in Larger Vehicles - for larger vehicles, a hierarchical network management arrangement is useful. Centralized network management is performed from a fixed NOC while local management is performed locally from within the

Herberg, et al.

RFC 7939
Standards Track

The NHDP-MIB
[Page 68]

August 2016

vehicles. Standards-based methods for configuration, monitoring, and fault management are necessary for this deployment option.

10. IANA Considerations

The MIB module in this document uses the following IANA-assigned OBJECT IDENTIFIER value recorded in the SMI Numbers registry:

$\begin{array}{ll}\text { Description } & \text { OBJECT IDENTIFIER value } \\ \text { NHDP-MIB } & \{\text { mib-2 213 }\end{array}$

11. References

11.1. Normative References

[RFC2119] Bradner, S., "Key words for use in RFCs to Indicate Requirement Levels", BCP 14, RFC 2119, DOI $10.17487 /$ RFC2119, March 1997 , <http://www.rfc-editor.org/info/rfc2119>.

[RFC2578] MCCloghrie, K., Ed., Perkins, D., Ed., and J. Schoenwaelder, Ed., "Structure of Management Information Version 2 (SMIV2)", STD 58, RFC 2578, DOI 10.17487/RFC2578, April 1999, <http://www.rfc-editor.org/info/rfc2578>.

[RFC2579] MCCloghrie, K., Ed., Perkins, D., Ed., and J. 
Schoenwaelder, Ed., "Textual Conventions for SMIv2", STD 58, RFC 2579, DOI 10.17487/RFC2579, April 1999, <http://www.rfc-editor.org/info/rfc2579>.

[RFC2580] MCCloghrie, K., Ed., Perkins, D., Ed., and J. Schoenwaelder, Ed., "Conformance Statements for SMIv2", STD 58, RFC 2580, DOI 10.17487/RFC2580, April 1999, <http://www.rfc-editor.org/info/rfc2580>.

[RFC2863] MCCloghrie, K. and F. Kastenholz, "The Interfaces Group MIB", RFC 2863, DOI 10.17487/RFC2863, June 2000, <http://www.rfc-editor.org/info/rfc2863>.

[RFC3414] Blumenthal, U. and B. Wijnen, "User-based Security Model (USM) for version 3 of the Simple Network Management Protocol (SNMPV3)", STD 62, RFC 3414, DOI $10.17487 / \mathrm{RFC}_{414}$, December 2002, <http: / /www.rfc-editor.org/info/rfc3414>.

Herberg, et al. RFC 7939
Standards Track

The NHDP-MIB
[Page 69]

August 2016

[RFC3418] Presuhn, R., Ed., "Management Information Base (MIB) for the Simple Network Management Protocol (SNMP)", STD 62, RFC 3418, DOI 10.17487/RFC3418, December 2002, <http://www.rfc-editor.org/info/rfc3418>.

[RFC3826] Blumenthal, U., Maino, F., and K. McCloghrie, "The Advanced Encryption Standard (AES) Cipher Algorithm in the SNMP User-based Security Model", RFC 3826, DOI $10.17487 / \mathrm{RFC} 3826$, June 2004, <http: //www.rfc-editor.org/info/rfc3826>.

[RFC4001] Daniele, M., Haberman, B., Routhier, S., and J. Schoenwaelder, "Textual Conventions for Internet Network Addresses", RFC 4001, DOI 10.17487/RFC4001, February 2005, <http: / /www.rfc-editor.org/info/rfc4001>.

[RFC5591] Harrington, D. and W. Hardaker, "Transport Security Model for the Simple Network Management Protocol (SNMP)", STD 78, RFC 5591, DOI 10.17487/RFC5591, June 2009, <http://www.rfc-editor.org/info/rfc5591>.

[RFC5592] Harrington, D., Salowey, J., and W. Hardaker, "Secure Shell Transport Model for the Simple Network Management Protocol (SNMP)", RFC 5592, DOI 10.17487/RFC5592, June 2009, <http://wWw.rfc-editor.org/info/rfc5592>.

[RFC6130] Clausen, T., Dearlove, C., and J. Dean, "Mobile Ad Hoc Network (MANET) Neighborhood Discovery Protocol (NHDP)", RFC 6130, DOI 10.17487/RFC6130, April 2011, <http://wWw.rfc-editor.org/info/rfc6130>.

[RFC6340] Presuhn, R., "Textual Conventions for the Representation of Floating-Point Numbers", RFC 6340, 
DOI $10.17487 /$ RFC6340, August 2011,

<http://www.rfc-editor.org/info/rfc6340>.

[RFC6353] Hardaker, W., "Transport Layer Security (TLS) Transport Model for the Simple Network Management Protocol (SNMP)", STD 78, RFC 6353, DOI 10.17487/RFC6353, July 2011, <http://www.rfc-editor.org/info/rfc6353>.

[RFC7466] Dearlove, C. and T. Clausen, "An Optimization for the Mobile Ad Hoc Network (MANET) Neighborhood Discovery Protocol (NHDP)", RFC 7466, DOI 10.17487/RFC7466, March 2015, <http://wWw.rfc-editor.org/info/rfc7466>.

Herberg, et al. RFC 7939
Standards Track

The NHDP-MIB
[Page 70]

August 2016

\subsection{Informative References}

[RFC3410] Case, J., Mundy, R., Partain, D., and B. Stewart, "Introduction and Applicability Statements for InternetStandard Management Framework", RFC 3410, DOI $10.17487 /$ RFC3410, December 2002, <http://www.rfc-editor.org/info/rfc3410>.

[RFC3411] Harrington, D., Presuhn, R., and B. Wijnen, "An Architecture for Describing Simple Network Management Protocol (SNMP) Management Frameworks", STD 62, RFC 3411, DOI 10.17487/RFC3411, December 2002, <http: //www.rfc-editor.org/info/rfc3411>.

[RFC4750] Joyal, D., Ed., Galecki, P., Ed., Giacalone, S., Ed., Coltun, R., and F. Baker, "OSPF Version 2 Management Information Base", RFC 4750, DOI 10.17487/RFC4750, December 2006 , <http://Www.rfc-editor.org/info/rfc4750>.

[RFC5148] Clausen, T., Dearlove, C., and B. Adamson, "Jitter Considerations in Mobile Ad Hoc Networks (MANETs)", RFC 5148, DOI 10.17487/RFC5148, February 2008, <http://www.rfc-editor.org/info/rfc5148>.

[RFC6779] Herberg, U., Cole, R., and I. Chakeres, "Definition of Managed Objects for the Neighborhood Discovery Protocol", RFC 6779, DOI 10.17487/RFC6779, October 2012, <http: / /www.rfc-editor.org/info/rfc6779>. 
Herberg, et al.

RFC 7939
Standards Track

The NHDP-MIB
[Page 71]

August 2016

\section{Acknowledgements}

The authors wish to thank Benoit Claise, Elwyn Davies, Justin Dean, Adrian Farrel, Joel Halpern, Michael MacFaden, Al Morton, and Thomas Nadeau for their detailed reviews and insightful comments regarding RFC 6779 and this document.

This MIB document uses the template authored by D. Harrington, which is based on contributions from the MIB Doctors, especially Juergen Schoenwaelder, Dave Perkins, C.M. Heard, and Randy Presuhn.

\section{Authors' Addresses}

Ulrich Herberg

United States of America

Email: ulrich@herberg.name

URI: http://wWw.herberg.name/

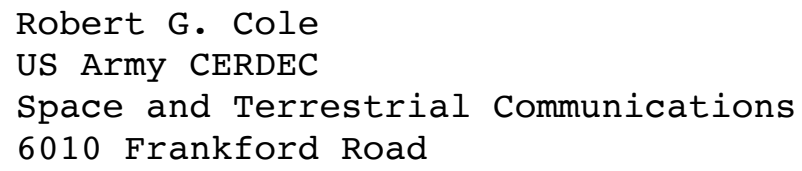


Phone: +33660589349

Email: T.Clausendcomputer.org

URI : http://WWW.ThomasClausen.org/

Herberg, et al.

Standards Track

[Page 72] 